\title{
Dynamical Casimir Effect in a small compact manifold for the Maxwell vacuum.
}

\author{
Ariel R. Zhitnitsky \\ Department of Physics $\&$ Astronomy, University of British Columbia, Vancouver, B.C. V6T 1Z1, Canada
}

\begin{abstract}
We study novel type of contributions to the partition function of the Maxwell system defined on a small compact manifold $\mathbb{M}$ such as torus. These new terms can not be described in terms of the physical propagating photons with two transverse polarizations. Rather, these novel contributions emerge as a result of tunnelling events when transitions occur between topologically different but physically identical vacuum winding states. These new terms give an extra contribution to the Casimir pressure, yet to be measured.

We argue that if the same system is considered in the background of a small external timedependent magnetic field, than there will be emission of photons from the vacuum, similar to the Dynamical Casimir Effect (DCE) when real particles are radiated from the vacuum due to the timedependent boundary conditions. The difference with conventional DCE is that the dynamics of the vacuum in our system is not related to the fluctuations of the conventional degrees of freedom, the virtual photons. Rather, the radiation in our case occurs as a result of tunnelling events between topologically different but physically identical $|k\rangle$ sectors in a time -dependent background. We comment on relation of this novel effect with the well-known, experimentally observed, and theoretically understood phenomena of the persistent currents in normal metal rings. We also comment on possible cosmological applications of this effect.
\end{abstract}

PACS numbers: 11.15.-q, 11.15.Kc, 11.15.Tk

\section{INTRODUCTION. MOTIVATION.}

The main motivation for present studies is as follows. It has been recently argued [1-3] that if free Maxwell theory (without any interactions with charged particles) is defined on a small compact manifold than some novel terms in the partition function will emerge. These terms are not related to the propagating photons with two transverse physical polarizations, which are responsible for the conventional Casimir effect (CE)[4]. Rather, these novel terms occur as a result of tunnelling events between topologically different but physically identical $|k\rangle$ topological sectors. These states play no role when the system is defined in infinitely large Minkowski space-time $\mathbb{R}_{1,3}$. But these states become important when the system is defined on a small compact manifold. Without loosing any generality we shall call this manifold $\mathbb{M}$, it could be the four-torus $\mathbb{T}^{4}$, or it could be any other compact manifold with a non-trivial mapping $\pi_{1}[U(1)]=\mathbb{Z}$. Precisely this non-trivial mapping for the Maxwell $U(1)$ gauge theory implies the presence of the topological sectors $|k\rangle$ which play the key role in our discussions. The corresponding phenomenon was coined as the topological Casimir Effect (TCE).

In particular, it has been explicitly shown in [1] that these novel terms in the topological portion of the partition function $\mathcal{Z}_{\text {top }}$ lead to a fundamentally new contributions to the Casimir vacuum pressure, which can not be expressed in terms of conventional propagating physical degrees of freedom. Furthermore, the $\mathcal{Z}_{\text {top }}$ shows many features of topologically ordered systems, which were initially introduced in context of condensed matter (CM) systems, see recent reviews [5-9]. In particular, $\mathcal{Z}_{\text {top }}$ demonstrates the degeneracy of the system which can not be described in terms of any local operators [2].
Instead, such a degeneracy can be formulated in terms of some non-local operators, see few comments on this classification in Appendix A. Furthermore, the infrared physics of the system can be studied in terms of auxiliary topological non-propagating fields [3] precisely in the same way as a topologically ordered system can be analyzed in terms of the Berry's connection (which is also emergent rather than a fundamental field). Furthermore, the corresponding expectation value of the auxiliary topological filed determines the phase of the system.

As we review in section II, the relevant vacuum fluctuations which saturate the topological portion of the partition function $\mathcal{Z}_{\text {top }}$ are formulated in terms of topologically non-trivial boundary conditions. These configurations satisfy the periodic boundary conditions on gauge field up to a large gauge transformation such that the tunnelling transitions occur between physically identical but topologically distinct $|k\rangle$ sectors. Precisely these field configurations generate an extra Casimir vacuum pressure in the system. What happens to this complicated vacuum structure when the system is placed into the background of external constant magnetic field $B_{\text {ext }}^{z}$ ? The answer on this question is known: the corresponding partition function $\mathcal{Z}_{\text {top }}$ as well as all observables, including the topological part of the Casimir pressure, are highly sensitive to small magnetic field and demonstrate the $2 \pi$ periodicity with respect to magnetic flux represented by parameter $\theta_{\text {eff }} \equiv e S B_{\text {ext }}^{z}$ where $S$ is the $x y$ area of the system $\mathbb{T}^{4}$. This sensitivity to external magnetic field is a result of the quantum interference of the external filed $B_{\text {ext }}^{z}$ with topological quantum fluctuations describing the tunnelling transitions between $|k\rangle$ sectors. This strong "quantum" sensitivity of the TCE should be contrasted with conventional Casimir forces which are practically unaltered by the external field due to very 
strong suppression $\sim B_{\text {ext }}^{2} / m_{e}^{4}$, see [1] for the details.

The main goal of the present work is to the study the dynamics of these vacuum fluctuations in the presence of a time-dependent magnetic field $B_{\text {ext }}^{z}(t)$. We would like to argue in the present work that there will be a radiation of real photons emitted from the vacuum (described by the partition function $\left.\mathcal{Z}_{\mathrm{top}}\left[B_{\mathrm{ext}}^{z}(t)\right]\right)$ as a result of this time dependent external source $B_{\text {ext }}^{z}(t)$.

A simple intuitive picture of this emission can be explained as follows. Imagine that we study conventional $\mathrm{CE}$ with static metallic plates. When these plates move or fluctuate, there will be emission from the vacuum, which is well known and well studied phenomenon known as the Dynamical Casimir Effect (DCE), see original papers [10] and reviews [11]. The DCE has been observed in recent experiments with superconducting circuits [12]. Basically, the virtual photons which are responsible for conventional Casimir pressure may become the real photons when the plate is moving or fluctuating.

The novel effect which is the subject of this work is that the topological configurations describing the tunnelling transitions between $|k\rangle$ sectors will be also modified when there is a time dependent external influence on the system. This time dependent impact on the system can be realized by moving the plates of the original manifold $\mathbb{M}$, in close analogy with DCE. The time-dependent impact may also enter the system through the quantum interference of the external field with topological configurations saturating the partition function $\mathcal{Z}_{\text {top }}$. Such quantum interference, as we mentioned above, is practically absent in conventional $\mathrm{CE}$ but is order of one in the TCE. Therefore, this quantum interference gives us a unique chance to manipulate with the Maxwell vacuum defined on $\mathbb{M}$ using a time-dependent external electromagnetic source. This effect leads to the production of the real photons with transverse polarizations emitted from the topological quantum vacuum configurations saturating the partition function $\mathcal{Z}_{\text {top }}$ in the presence of time-dependent magnetic field $B_{\text {ext }}^{z}(t)$.

As this effect is very novel and quite counter-intuitive, we would like to present one more additional explanation supporting our claim that there will be emission of real photons from the Maxwell vacuum when the systems (described by the partition function $\mathcal{Z}_{\text {top }}$ ) is placed into the background of time-dependent source $B_{\text {ext }}^{z}(t)$.

Our second explanation goes as follows. The topological configurations which describe the tunnelling transitions are formulated in terms of the boundary conditions on gauge field up to a large gauge transformations. These boundary conditions correspond to some persistent fluctuating currents which can flow along the metallic boundaries corresponding to the edges of $\mathbb{M}$. In fact, the possibility that such persistent current may occur in small rings with topology $\mathbb{S}^{1}$ have been theoretically predicted long ago [13], though with very different motivation from the one advocated in present work. Furthermore, the corresponding persistent non-dissipating currents in different materials have been experimentally observed in small rings $\mathbb{S}^{1}$, see reviews [14]. Our comment here is that similar currents flowing along the rings of $\mathbb{S}^{1}$ which represents the boundary of $\mathbb{M}$ can be interpreted as a result of topological vacuum configurations intimately related to Aharonov Bohm phases when the system is defined on a topologically nontrivial manifold. We elaborate on this connection (between the persistent currents and our description in terms of the topological vacuum configurations) further in the text.

The only comment we would like to make here is as follows. The persistent clockwise and anti-clockwise currents cancel each other in case of vanishing external magnetic field. This cancellation does not hold in the presence of a time-independent external magnetic field $B_{\text {ext }}^{z}$ perpendicular to the ring $\mathbb{S}^{1}$, in which case the persistent current $I_{0}$ will be generated. One can view this system as generation of a static magnetic moment $m_{\text {ind }}^{z}=I_{0} S$. It is quite obvious now that if the external magnetic field $B_{\text {ext }}^{z}(t)$ becomes a time-dependent function, the corresponding induced magnetic moment $m_{\text {ind }}^{z}(t)$ also becomes a time-dependent function. The corresponding time-dependence in $m_{\text {ind }}^{z}(t)$ obviously implies that the system starts to radiate physical photons with typical angular distribution given by the magnetic dipole radiation. This radiation is ultimately related to the topological vacuum configurations describing the tunnelling transitions between $|k\rangle$ sectors. These vacuum configurations get modified in the presence of a time-dependent field $B_{\text {ext }}^{z}(t)$, which is precisely the source for the radiation of physical photons. In all respects the idea is very similar to DCE with the "only" difference is that the conventional virtual photons (responsible for the $\mathrm{CE}$ ) do not interfere with external magnetic field $B_{\mathrm{ext}}^{z}(t)$, while the topological vacuum instanton-like configurations (saturating the TCE) do. We coin the corresponding phenomenon of the emission of real photons from vacuum configurations saturating $\mathcal{Z}_{\text {top }}\left[B_{\text {ext }}^{z}(t)\right]$ in the presence of time dependent source $\left[B_{\text {ext }}^{z}(t)\right]$ the non-static (or dynamical) topological Casimir effect (TCE) to discriminate it from the conventional DCE.

The structure of our presentation is as follows. In next section II we review our previous results [1-3] on construction of the partition function $\mathcal{Z}_{\text {top }}$ describing the tunnelling transitions between $|k\rangle$ sectors. We also explain how this partition function is modified in the presence of external static magnetic field $B_{\text {ext }}^{z}$. After that in section III we generalize the construction to include the slow time-dependent fields, which allow us to compute the induced magnetic dipole moment of the system. This time dependent induced magnetic moment radiates real physical photons from vacuum. We also elaborate on relation of our construction with persistent currents in section III B. Finally, in sections III C, III D we make few simple numerical, order of magnitude estimates in order to get some insights on potential prospects of measuring the effect, which crucially depends on property of degeneracy of the system. As this feature of degeneracy is crucial for potential experimental studies of this effect, 
we make few comments on this property in Appendix A. The corresponding description of the system (when it is characterized by a global, rather than local observables) is quite different from conventional classification scheme when a system is characterized by an expectation value of a local operator.

Our conclusion is section IV where we speculate on possible relevance of this novel effect for cosmology when appropriate topology is $\pi_{3}[S U(3)]=\mathbb{Z}$ replacing the nontrivial mapping $\pi_{1}[U(1)]=\mathbb{Z}$ considered in present work for studying the Maxwell theory on a compact manifold. To be more concrete, we speculate that the de Sitter behaviour in inflationary epoch could be just inherent property of the topological sectors in QCD in expanding Universe, rather than a result of dynamics of some ad hoc dynamical field such as inflaton. The emission of real physical degrees of freedom from the inflationary vacuum in time dependent background (the so-called reheating epoch) in all respects is very similar to the effect considered in the present work when the real photons can be emitted from vacuum in the background of a time dependent magnetic field $B_{\text {ext }}^{z}(t)$.

\section{TOPOLOGICAL PARTITION FUNCTION}

Our goal here is to review the Maxwell system defined on a Euclidean 4-torus with sizes $L_{1} \times L_{2} \times L_{3} \times \beta$ in the respective directions. It provides the infrared (IR) regularization of the system. This IR regularization plays a key role in proper treatment of the topological terms which are related to tunnelling events between topologically distinct but physically identical $|k\rangle$ sectors.

\section{A. Construction}

We follow [1-3] in our construction of the partition function $\mathcal{Z}_{\text {top }}$ where it was employed for computation of the corrections to the Casimir effect due to these novel type of topological fluctuations. The crucial point is that we impose the periodic boundary conditions on gauge $A^{\mu}$ field up to a large gauge transformation. In what follows we simplify our analysis by considering a clear case with winding topological sectors $|k\rangle$ in the z-direction only. The classical instanton configuration in Euclidean space which describes the corresponding tunnelling transitions can be represented as follows:

$$
A_{\mathrm{top}}^{\mu}=\left(0,-\frac{\pi k}{e L_{1} L_{2}} x_{2}, \frac{\pi k}{e L_{1} L_{2}} x_{1}, 0\right),
$$

where $k$ is the winding number that labels the topological sector, and $L_{1}, L_{2}$ are the dimensions of the plates in the $\mathrm{x}$ and $\mathrm{y}$-directions respectively, which are assumed to be much larger than the distance between the plates $L_{3}$. This terminology ("instanton") is adapted from similar studies in 2d QED [1] where corresponding configuration in $A_{0}=0$ gauge describe the interpolation between pure gauge vacuum winding states $|k\rangle$. We use the same terminology and interpretation for $4 \mathrm{~d}$ case because (2) is the classical configuration saturating the partition function $\mathcal{Z}_{\text {top }}$ in close analogy with $2 \mathrm{~d}$ case as discussed in details in [1].This classical instanton-flux configuration satisfies the periodic boundary conditions up to a large gauge transformation, and provides a topological magnetic instanton-flux in the z-direction:

$$
\begin{aligned}
\vec{B}_{\text {top }} & =\vec{\nabla} \times \vec{A}_{\text {top }}=\left(0,0, \frac{2 \pi k}{e L_{1} L_{2}}\right), \\
\Phi & =e \int d x_{1} d x_{2} B_{\text {top }}^{z}=2 \pi k .
\end{aligned}
$$

The Euclidean action of the system is quadratic and has the following form

$$
\frac{1}{2} \int \mathrm{d}^{4} x\left\{\vec{E}^{2}+\left(\vec{B}+\vec{B}_{\mathrm{top}}\right)^{2}\right\}
$$

where $\vec{E}$ and $\vec{B}$ are the dynamical quantum fluctuations of the gauge field. We call the configuration given by eq. (1) the instanton-fluxes describing the tunnelling events between topological sectors $|k\rangle$. These configurations saturate the partition function (6) and should be interpreted as "large" quantum fluctuations which change the winding states $|k\rangle$, in contrast with "small" quantum fluctuations which are topologically trivial and expressed in terms of conventional virtual photons saturating the quantum portion of the partition function $\mathcal{Z}_{\text {quant }}$.

The key point is that the topological portion $\mathcal{Z}_{\text {top }}$ decouples from quantum fluctuations, $\mathcal{Z}=\mathcal{Z}_{\text {quant }} \times \mathcal{Z}_{\text {top }}$ such that the quantum fluctuations do not depend on topological sector $k$ and can be computed in topologically trivial sector $k=0$. Indeed, the cross term

$$
\int \mathrm{d}^{4} x \vec{B} \cdot \vec{B}_{\text {top }}=\frac{2 \pi k}{e L_{1} L_{2}} \int \mathrm{d}^{4} x B_{z}=0
$$

vanishes because the magnetic portion of quantum fluctuations in the $z$-direction, represented by $B_{z}=\partial_{x} A_{y}-$ $\partial_{y} A_{x}$, is a periodic function as $\vec{A}$ is periodic over the domain of integration. This technical remark in fact greatly simplifies our analysis as the contribution of the physical propagating photons is not sensitive to the topological sectors $k$. This is, of course, a specific feature of quadratic action (3), in contrast with non-abelian and non-linear gauge field theories where quantum fluctuations of course depend on topological $k$ sectors.

The classical action for configuration (2) takes the form

$$
\frac{1}{2} \int \mathrm{d}^{4} x \vec{B}_{\text {top }}^{2}=\frac{2 \pi^{2} k^{2} \beta L_{3}}{e^{2} L_{1} L_{2}}
$$

To simplify our analysis further in computing $\mathcal{Z}_{\text {top }}$ we consider a geometry where $L_{1}, L_{2} \gg L_{3}, \beta$ similar to construction relevant for the Casimir effect. In this case our system is closely related to $2 \mathrm{~d}$ Maxwell theory by dimensional reduction: taking a slice of the $4 \mathrm{~d}$ system in the $x y$-plane will yield precisely the topological features of 
the $2 \mathrm{~d}$ torus considered in great details in [1]. Furthermore, with this geometry our simplification (2) when we consider exclusively the magnetic instanton- fluxes in $z$ direction is justified as the corresponding classical action (5) assumes a minimal possible values. With this assumption we can consider very small temperature, but still we can not take a formal limit $\beta \rightarrow \infty$ in our final expressions as a result of our technical constraints in the system.

With these additional simplifications the topological partition function becomes [1-3] :

$$
\mathcal{Z}_{\text {top }}=\sqrt{\frac{2 \pi \beta L_{3}}{e^{2} L_{1} L_{2}}} \sum_{k \in \mathbb{Z}} e^{-\frac{2 \pi^{2} k^{2} \beta L_{3}}{e^{2} L_{1} L_{2}}}=\sqrt{\pi \tau} \sum_{k \in \mathbb{Z}} e^{-\pi^{2} \tau k^{2}}
$$

where we introduced the dimensionless parameter

$$
\tau \equiv 2 \beta L_{3} / e^{2} L_{1} L_{2}
$$

Formula (6) is essentially the dimensionally reduced expression for the topological partition function for $2 \mathrm{~d}$ Maxwell theory analyzed in [1]. One should note that the normalization factor $\sqrt{\pi \tau}$ which appears in eq. (6) does not depend on topological sector $k$, and essentially it represents our convention of the normalization $\mathcal{Z}_{\text {top }} \rightarrow 1$ in the limit $L_{1} L_{2} \rightarrow \infty$ which corresponds to a convenient set up for the Casimir -type experiments as discussed in $[1-3]$. The simplest way to demonstrate that $\mathcal{Z}_{\text {top }} \rightarrow 1$ in the limit $\tau \rightarrow 0$ is to use the dual representation (10), see below.

\section{B. External static magnetic field}

In this section we want to generalize our results for the Euclidean Maxwell system in the presence of the external magnetic field. Normally, in the conventional quantization of electromagnetic fields in infinite Minkowski space, there is no direct coupling between fluctuating vacuum photons and an external magnetic field as a consequence of linearity of the Maxwell system. The coupling with fermions generates a negligible effect $\sim \alpha^{2} B_{e x t}^{2} / m_{e}^{4}$ as the non-linear Euler-Heisenberg Effective Lagrangian suggests, see [1] for the details and numerical estimates. In contrast with conventional photons, the the external magnetic field does couple with topological fluctuations (2). It leads to the effects of order of unity as a result of interference of the external magnetic field with topological fluxes $k$.

The corresponding partition function can be easily constructed for external magnetic field $B_{z}^{\text {ext }}$ pointing along $z$ direction, as the crucial technical element on decoupling of the background fields from quantum fluctuations assumes the same form (4). In other words, the physical propagating photons with non-vanishing momenta are not sensitive to the topological $k$ sectors, nor to the external uniform magnetic field, similar to our discussions after eq.(4).
The classical action for configuration in the presence of the uniform static external magnetic field $B_{z}^{\text {ext }}$ therefore takes the form

$$
\frac{1}{2} \int \mathrm{d}^{4} x\left(\vec{B}_{\text {ext }}+\vec{B}_{\text {top }}\right)^{2}=\pi^{2} \tau\left(k+\frac{\theta_{\text {eff }}}{2 \pi}\right)^{2}
$$

where $\tau$ is defined by $(7)$ and the effective theta parameter $\theta_{\text {eff }} \equiv e L_{1} L_{2} B_{\text {ext }}^{z}$ is expressed in terms of the original external magnetic field $B_{\text {ext }}^{z}$. Therefore, the partition function in the presence of the uniform magnetic field can be easily reconstructed from (6), and it is given by $[1-3]$

$$
\mathcal{Z}_{\text {top }}\left(\tau, \theta_{\text {eff }}\right)=\sqrt{\pi \tau} \sum_{k \in \mathbb{Z}} \exp \left[-\pi^{2} \tau\left(k+\frac{\theta_{\text {eff }}}{2 \pi}\right)^{2}\right] .
$$

This system in what follows will be referred as the topological vacuum $(\mathcal{T} \mathcal{V})$ because the propagating degrees of freedom, the photons with two transverse polarizations, completely decouple from $\mathcal{Z}_{\text {top }}\left(\tau, \theta_{\text {eff }}\right)$.

The dual representation for the partition function is obtained by applying the Poisson summation formula such that (9) becomes

$$
\mathcal{Z}_{\text {top }}\left(\tau, \theta_{\text {eff }}\right)=\sum_{n \in \mathbb{Z}} \exp \left[-\frac{n^{2}}{\tau}+i n \cdot \theta_{\text {eff }}\right]
$$

Formula (10) justifies our notation for the effective theta parameter $\theta_{\text {eff }}$ as it enters the partition function in combination with integer number $n$. One should emphasize that integer number $n$ in the dual representation (10) is not the integer magnetic flux $k$ defined by eq. (2) which enters the original partition function (6). Furthermore, the $\theta_{\text {eff }}$ parameter which enters $(9,10)$ is not a fundamental $\theta$ parameter which is normally introduced into the Lagrangian in front of $\vec{E} \cdot \vec{B}$ operator. Rather, this parameter $\theta_{\text {eff }}$ should be understood as an effective parameter representing the construction of the $\left|\theta_{\text {eff }}\right\rangle$ state for each slice with non-trivial $\pi_{1}[U(1)]$ in four dimensional system. In fact, there are three such $\theta_{\text {eff }}^{M_{i}}$ parameters representing different slices and corresponding external magnetic fluxes. There are similar three $\theta_{\text {eff }}^{E_{i}}$ parameters representing the external electric fluxes as discussed in [2], such that total number of $\theta$ parameters classifying the system equals six, in agreement with total number of hyperplanes in four dimensions. We shall not elaborate on this classification in the present work. In this work we limit ourselves with a single $\theta_{\text {eff }}$ parameter entering (9), (10), and corresponding to the magnetic external field $B_{\text {ext }}^{z}$ pointing in $z$ direction.

\section{INDUCED MAGNETIC DIPOLE MOMENT AND $E \& M$ RADIATION}

The main goal of this section is to estimate the induced magnetic dipole moment of the system in a time dependent background. First, in section III A we derive a 


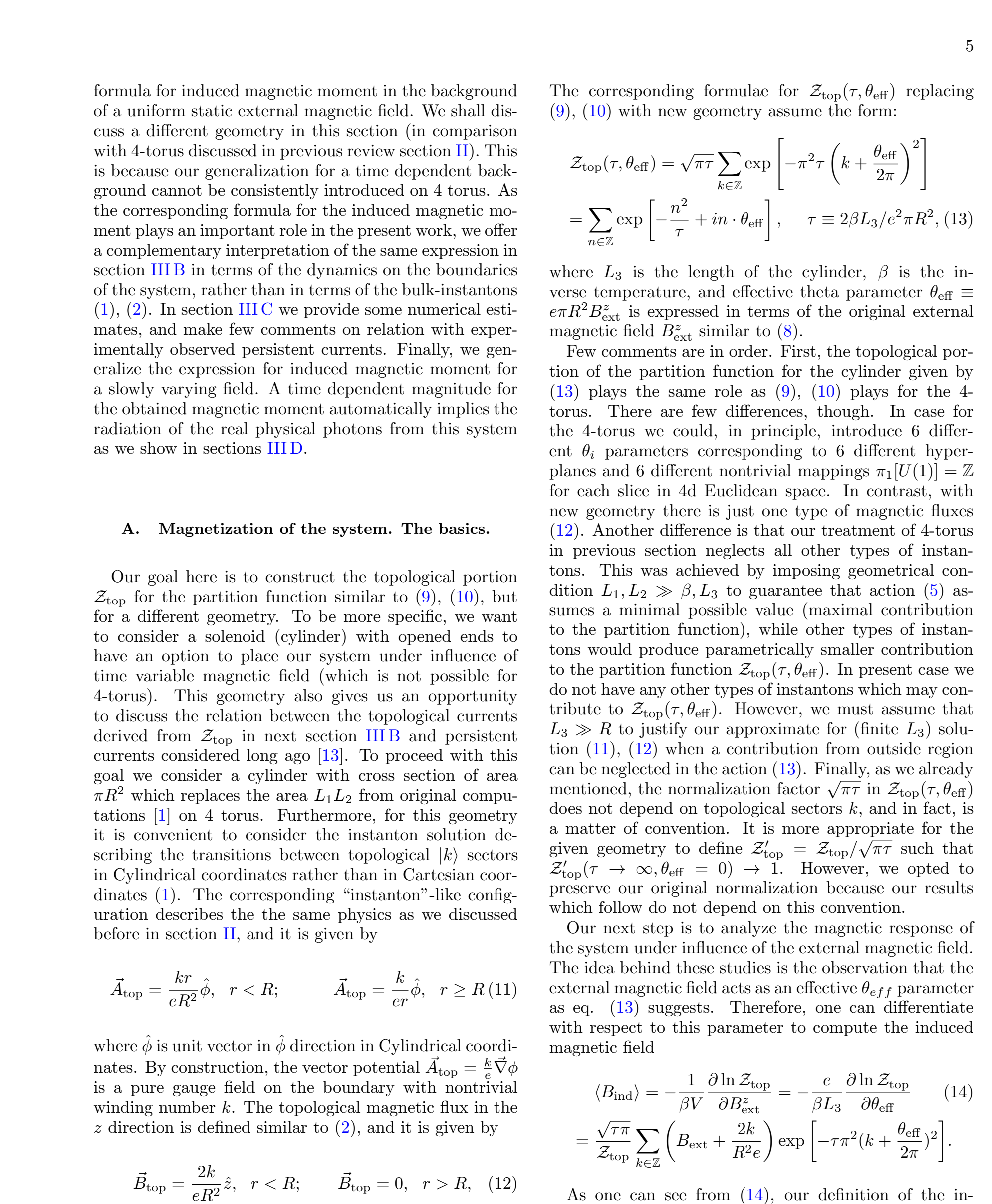

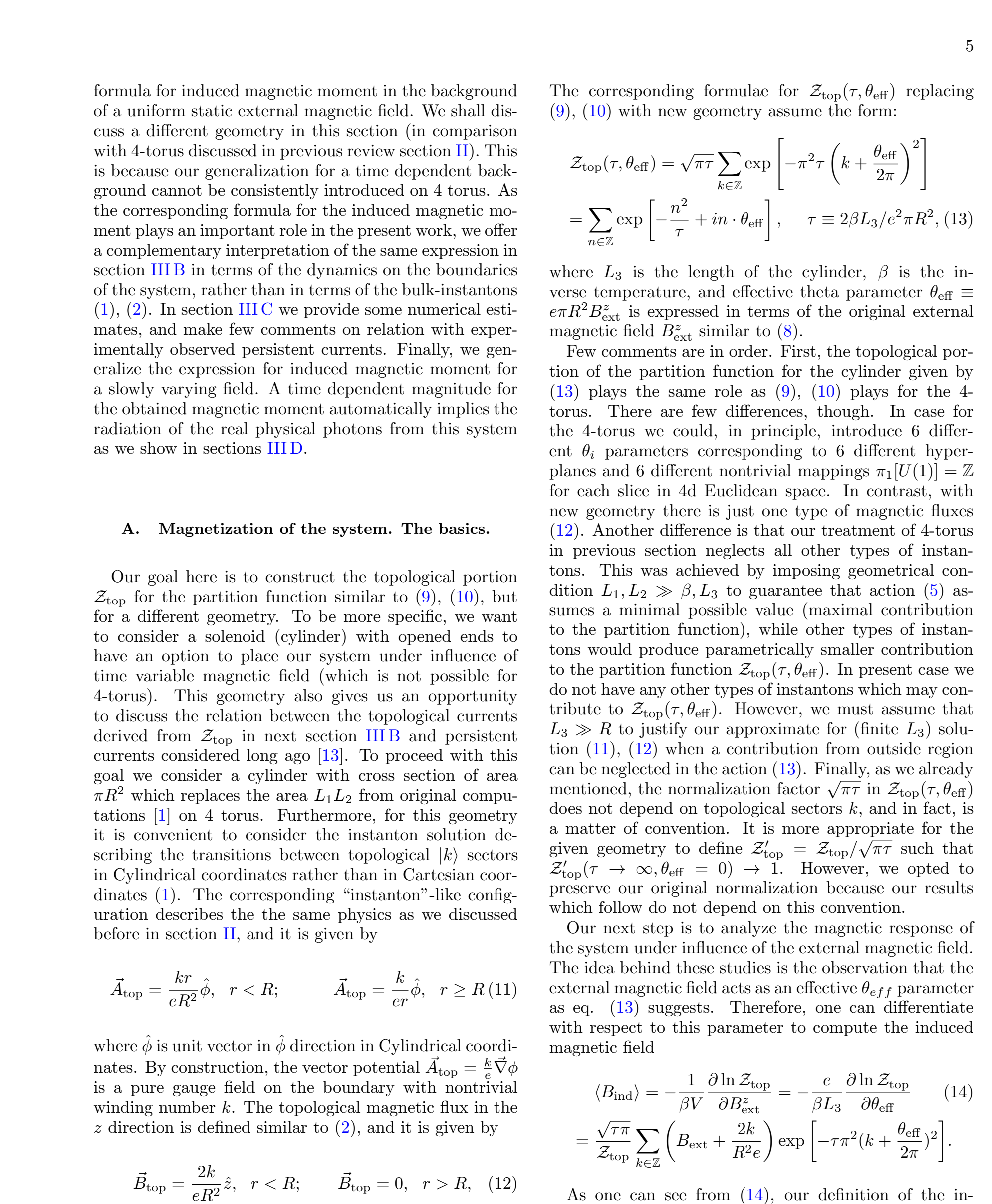

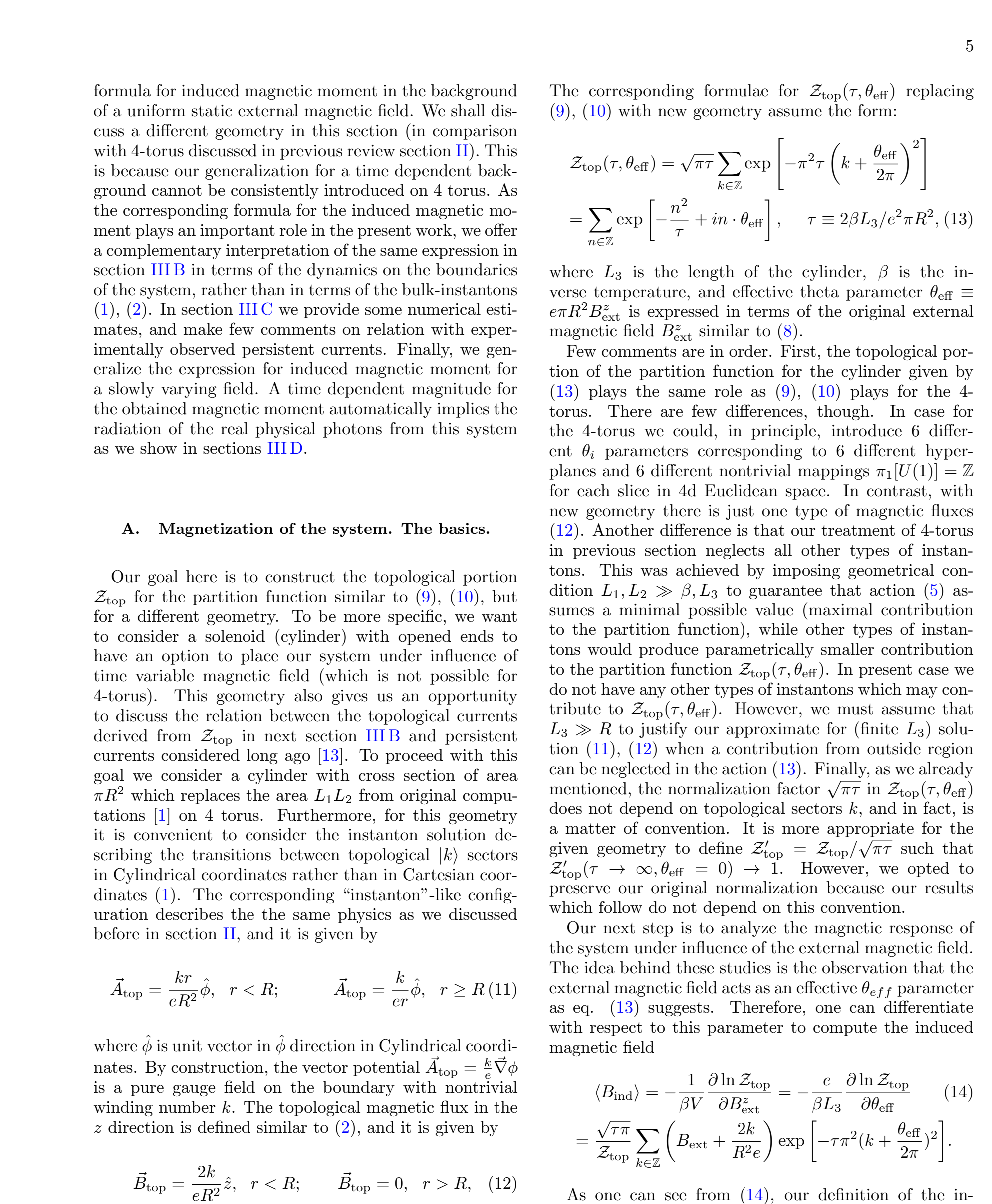

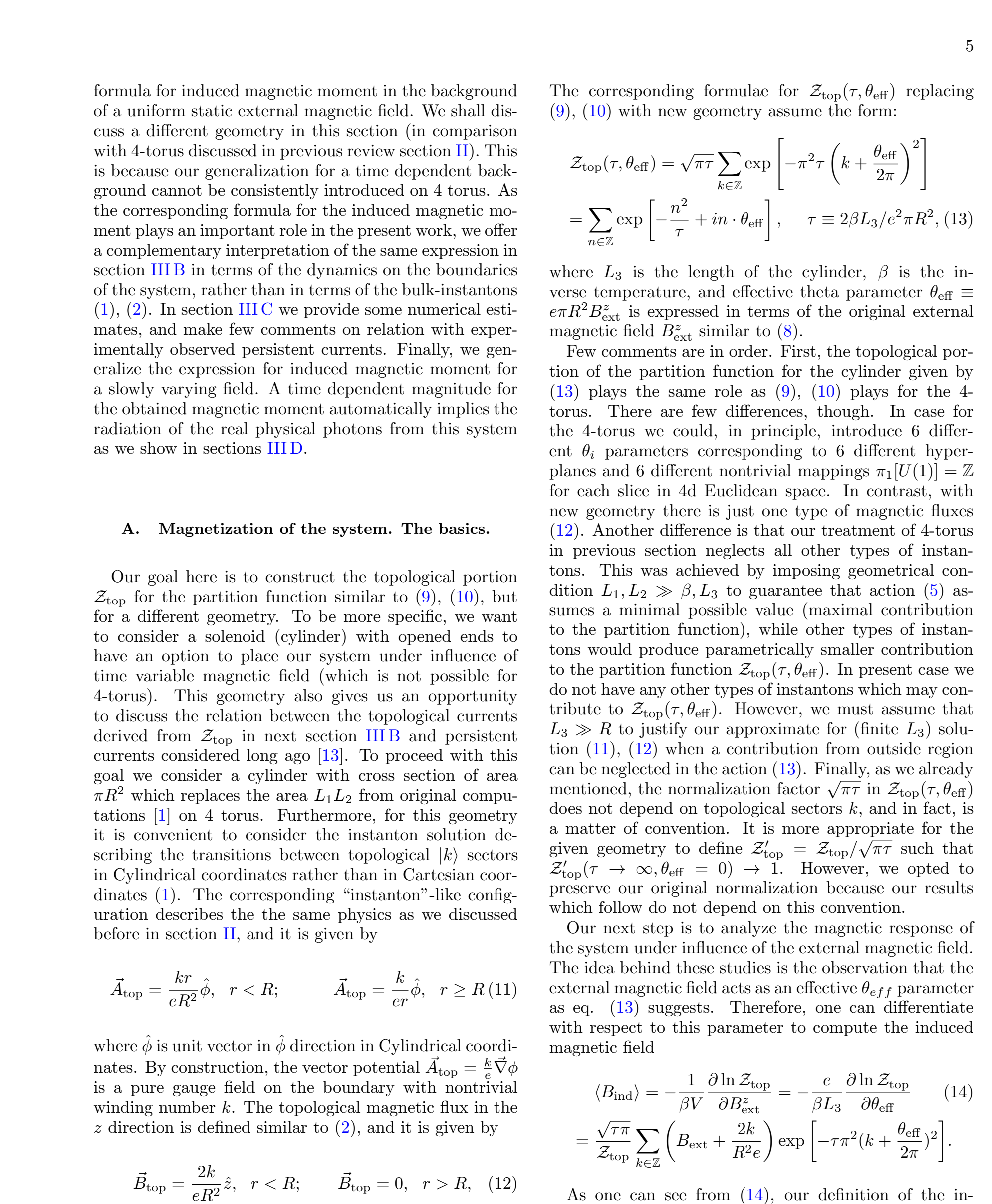

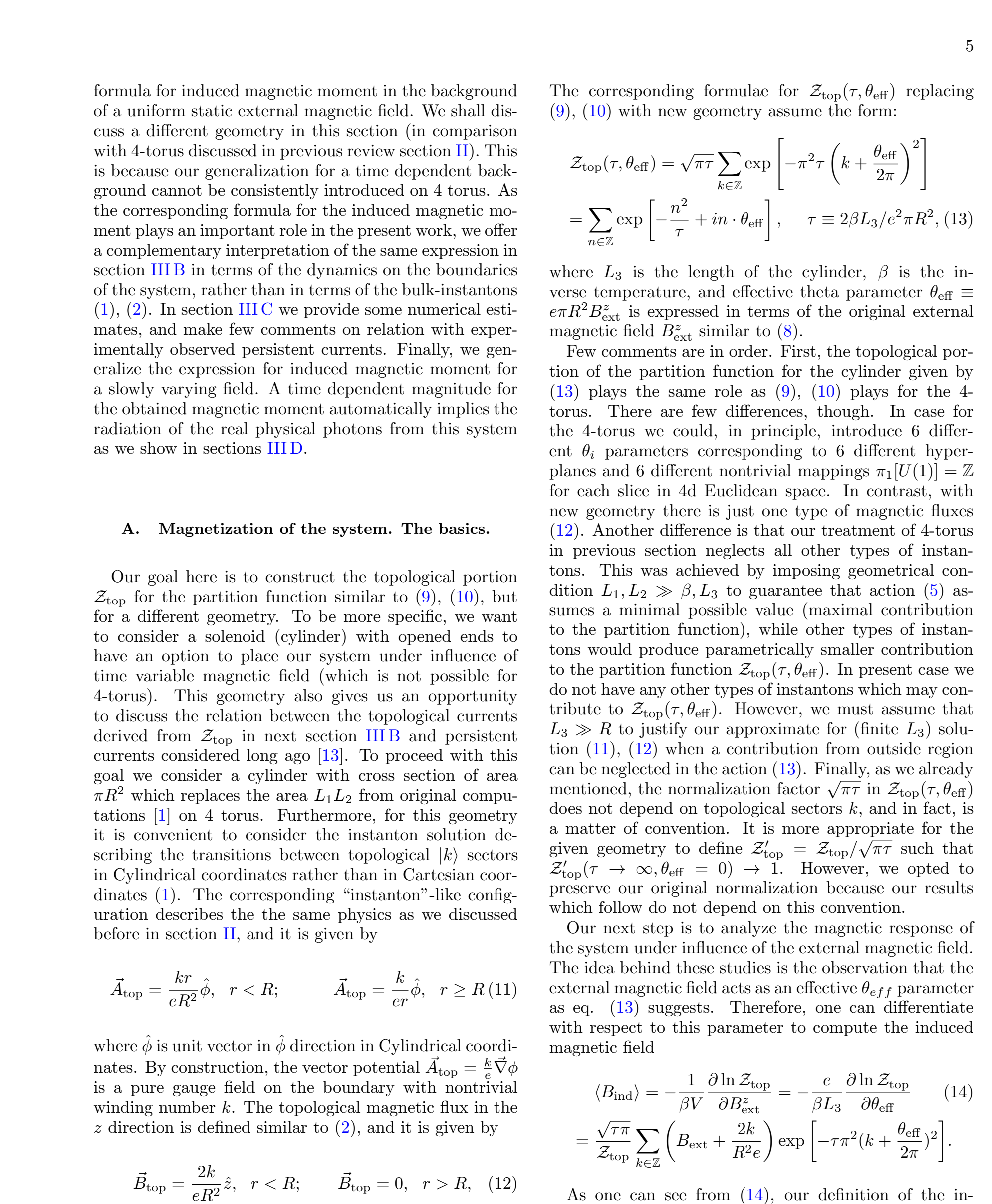

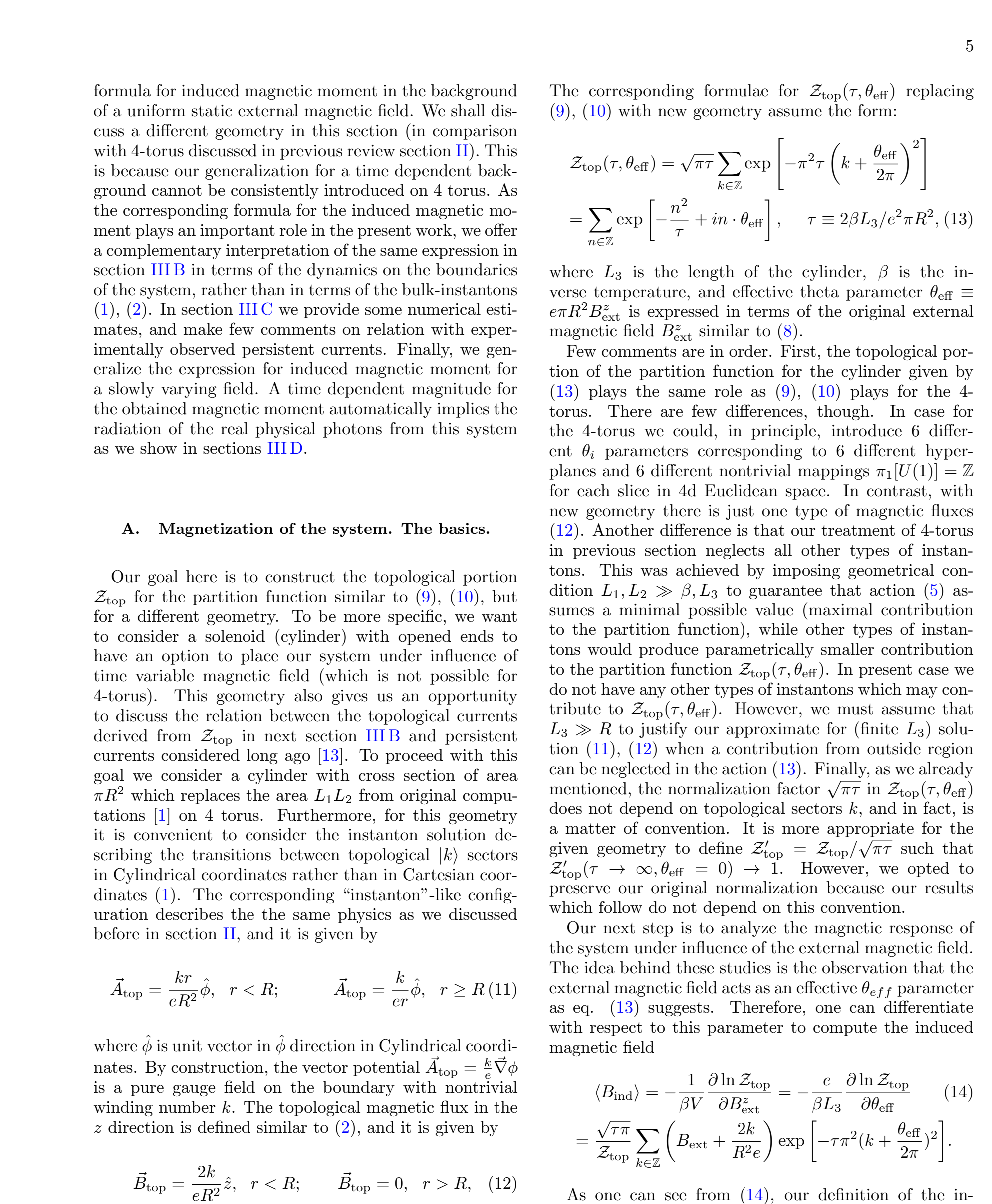

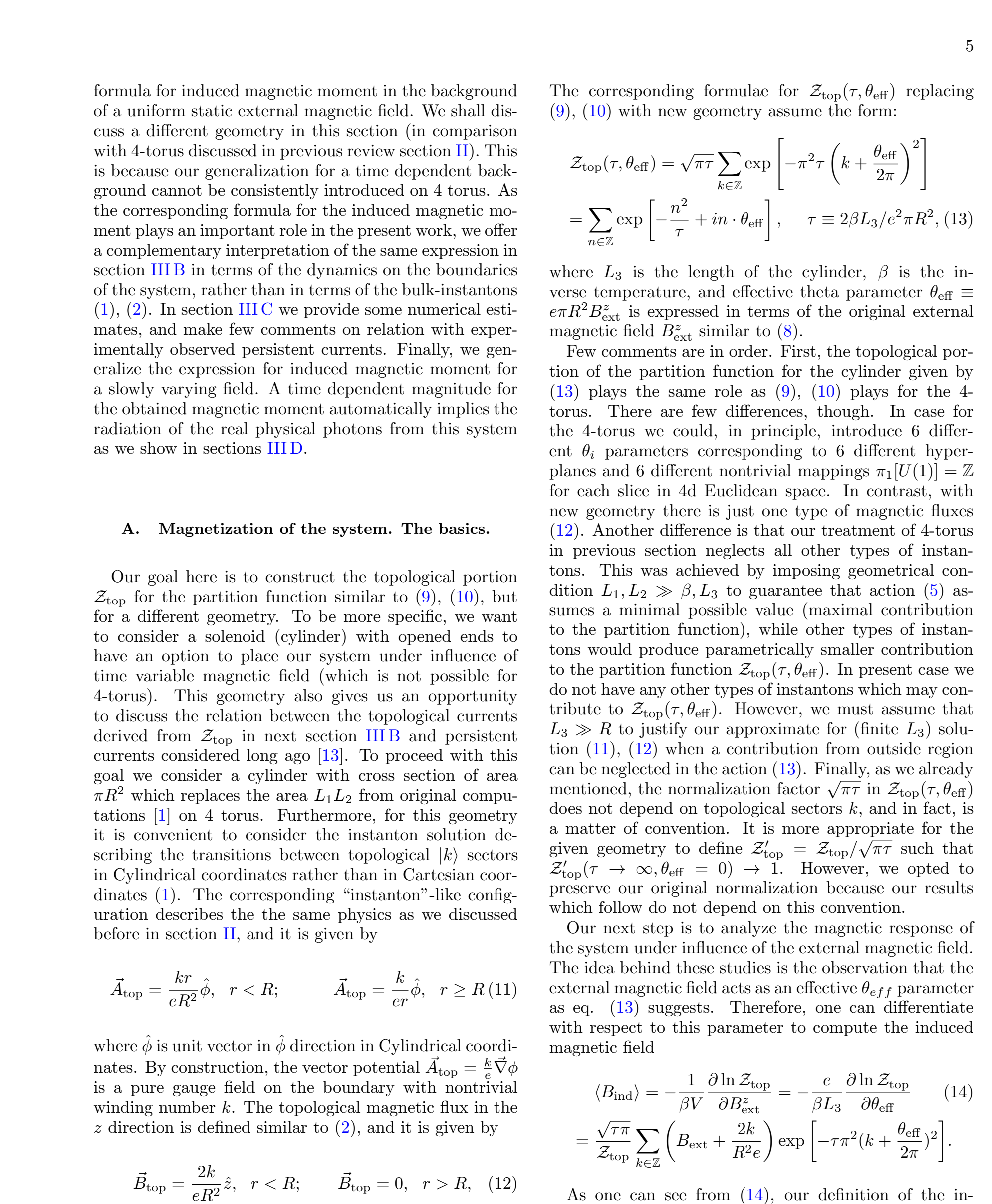

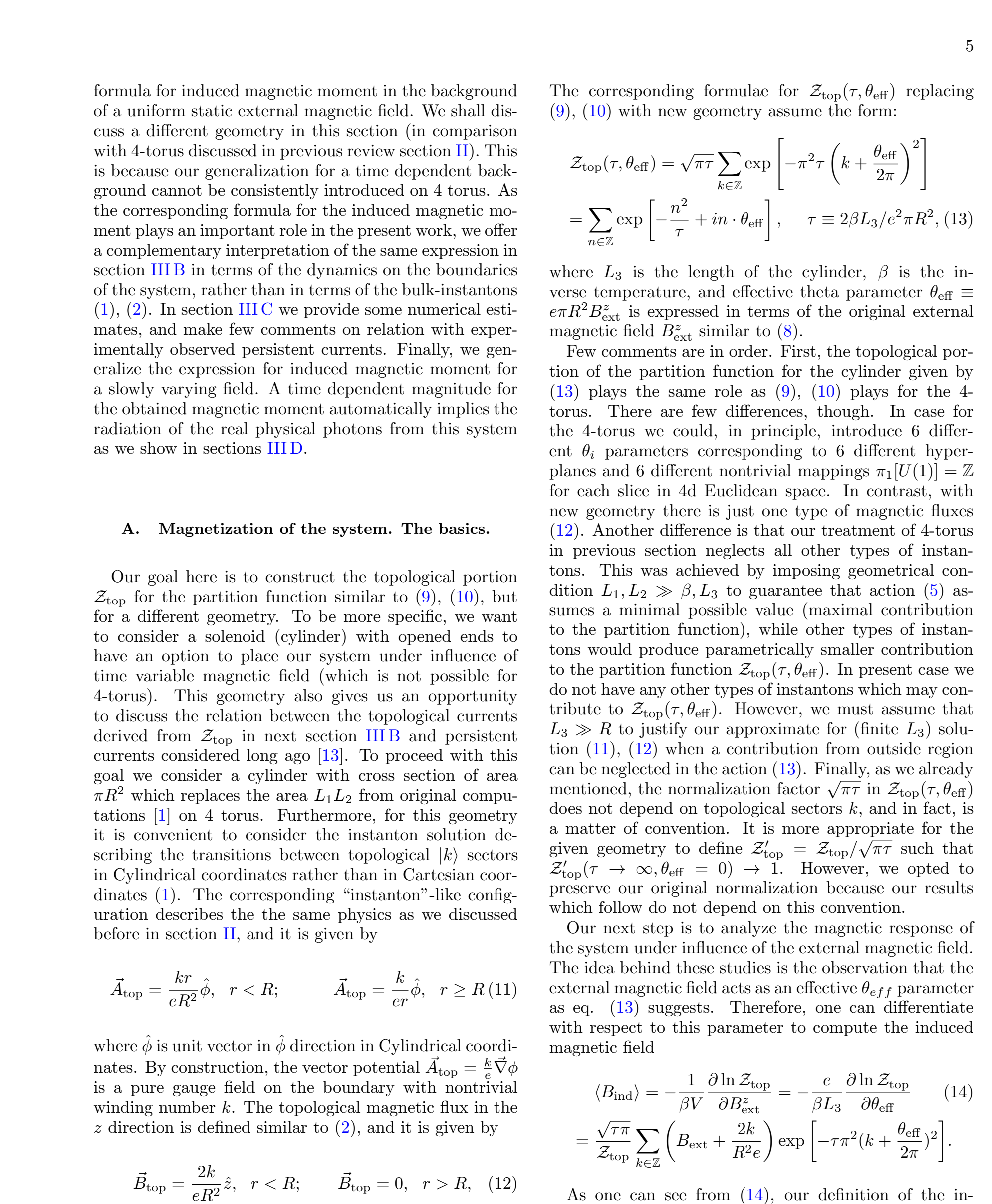

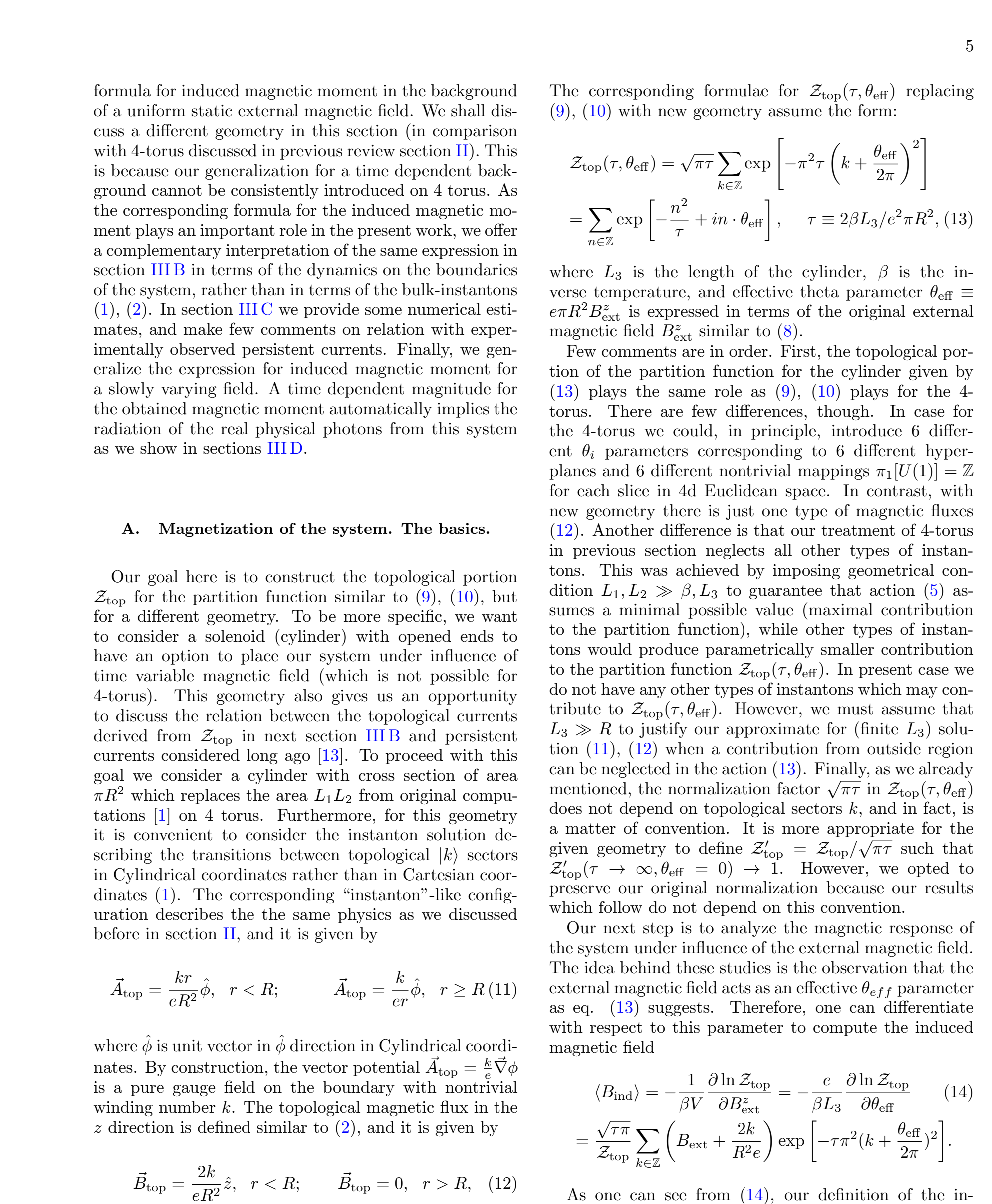

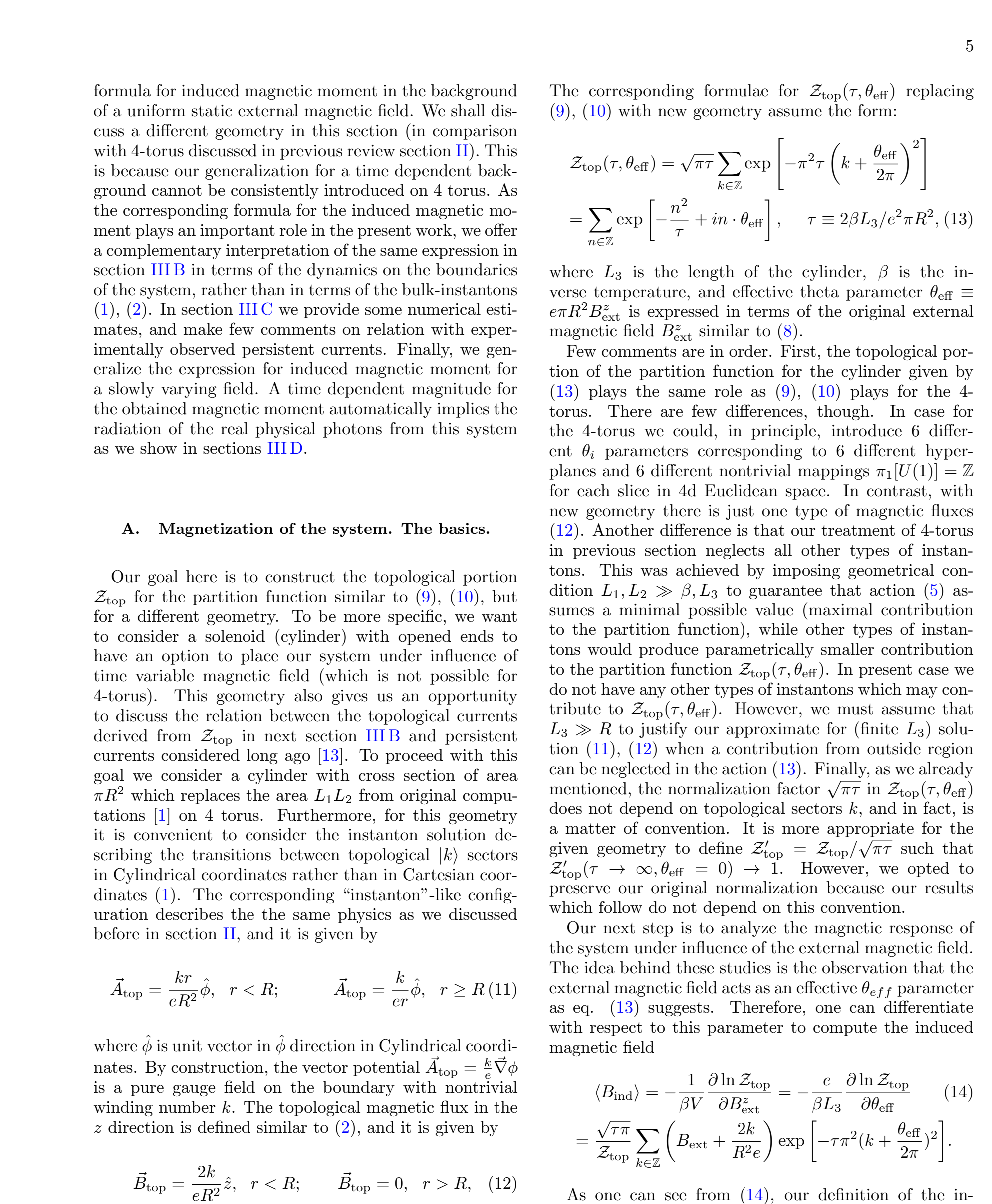

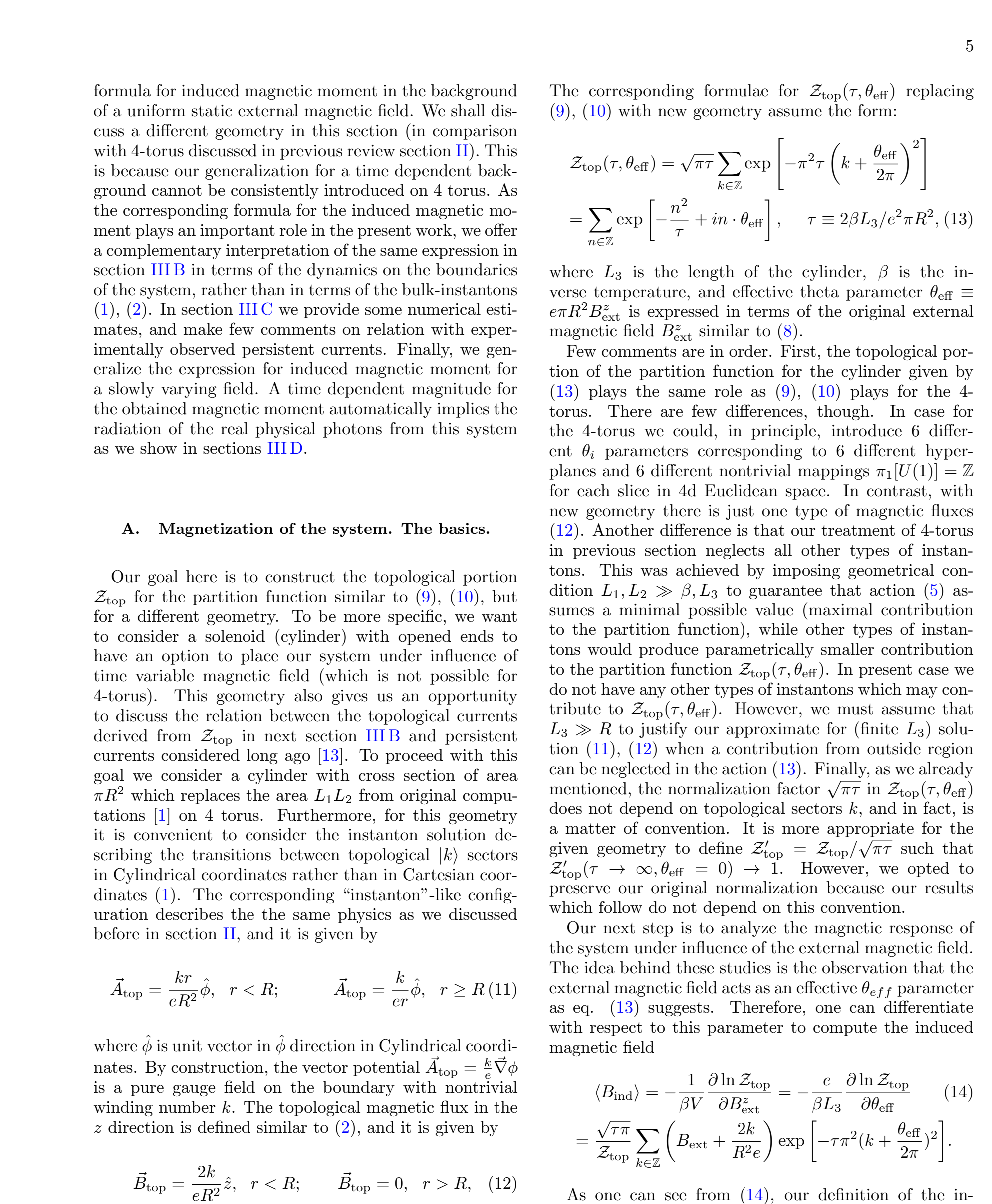

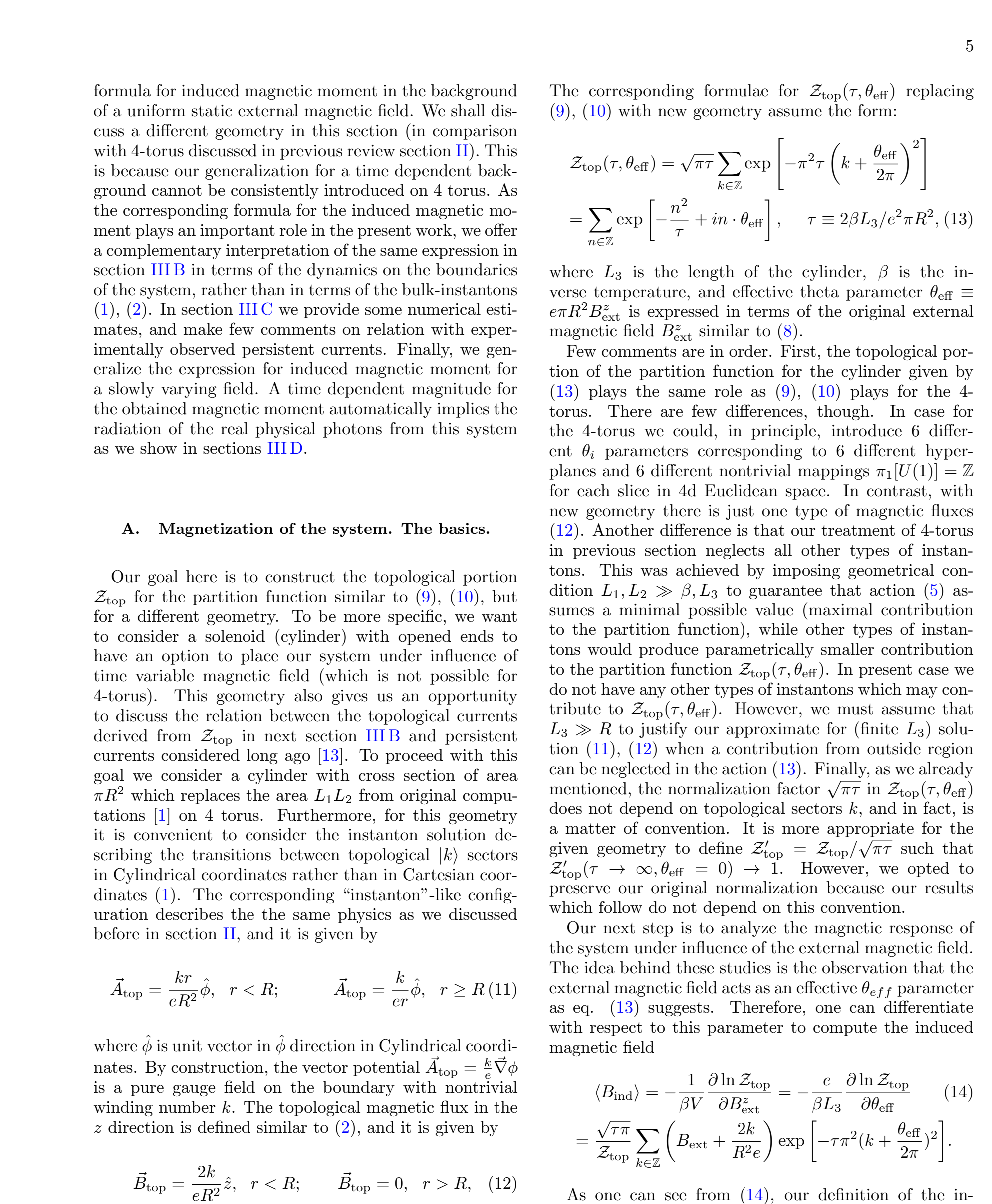

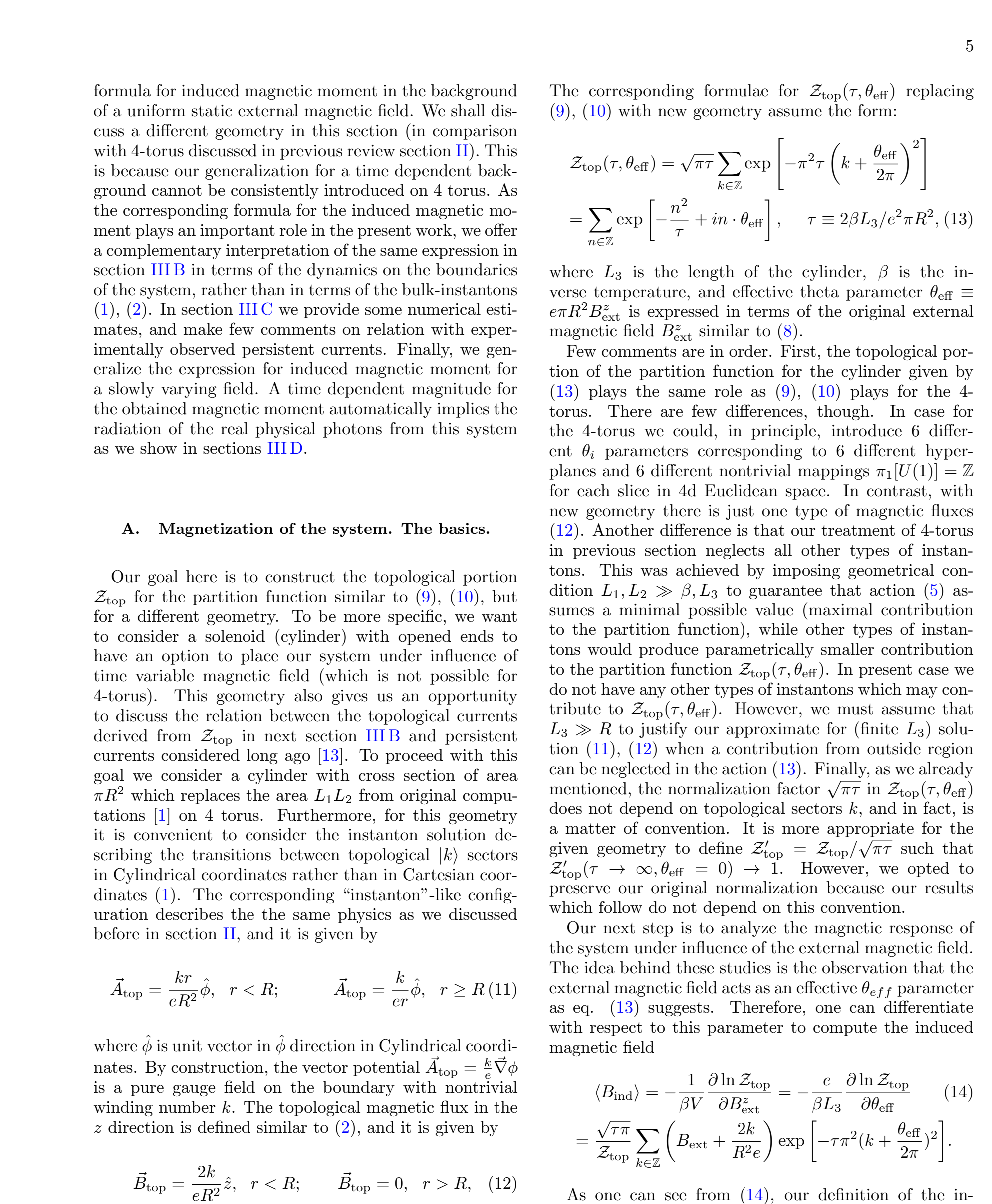

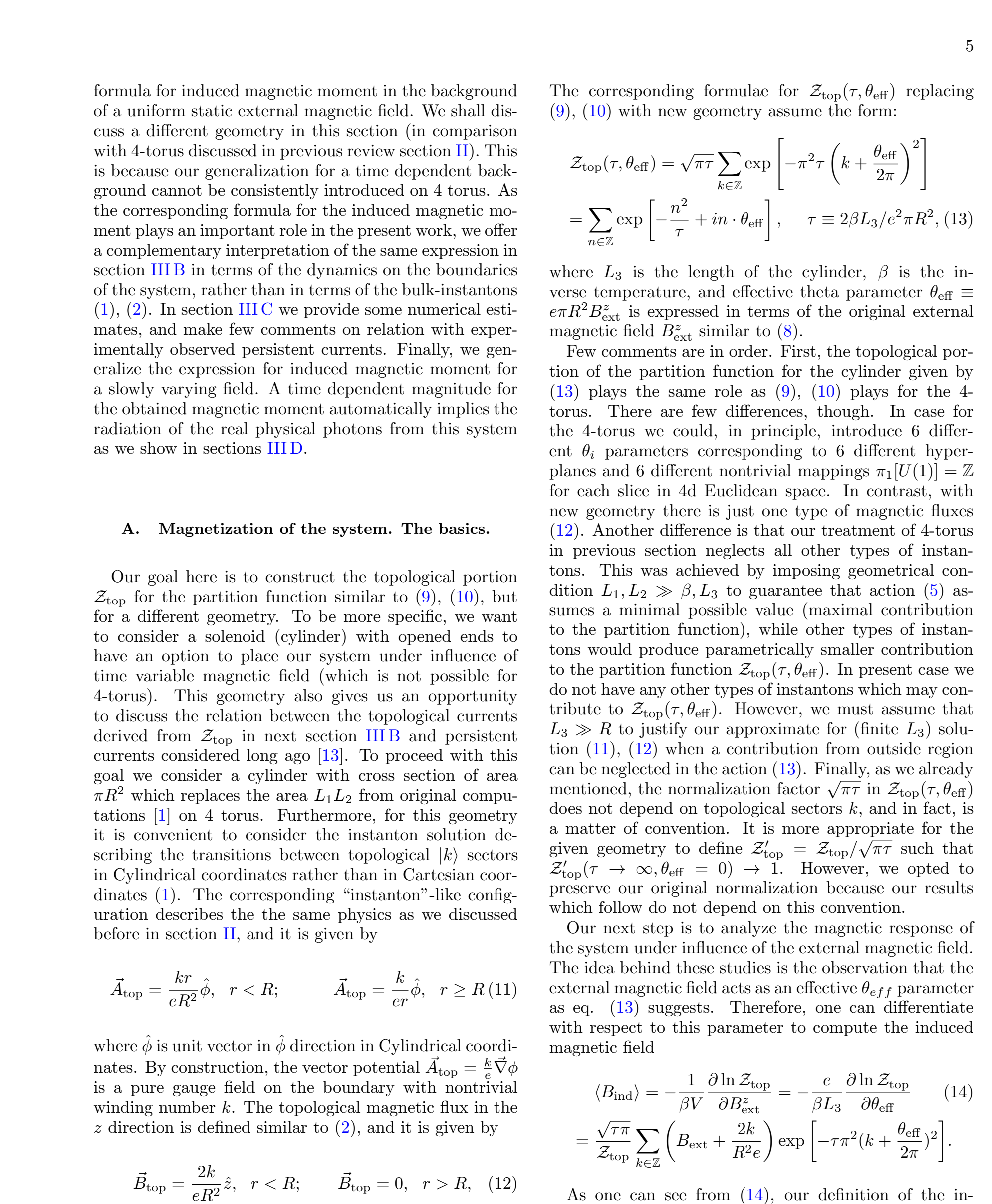

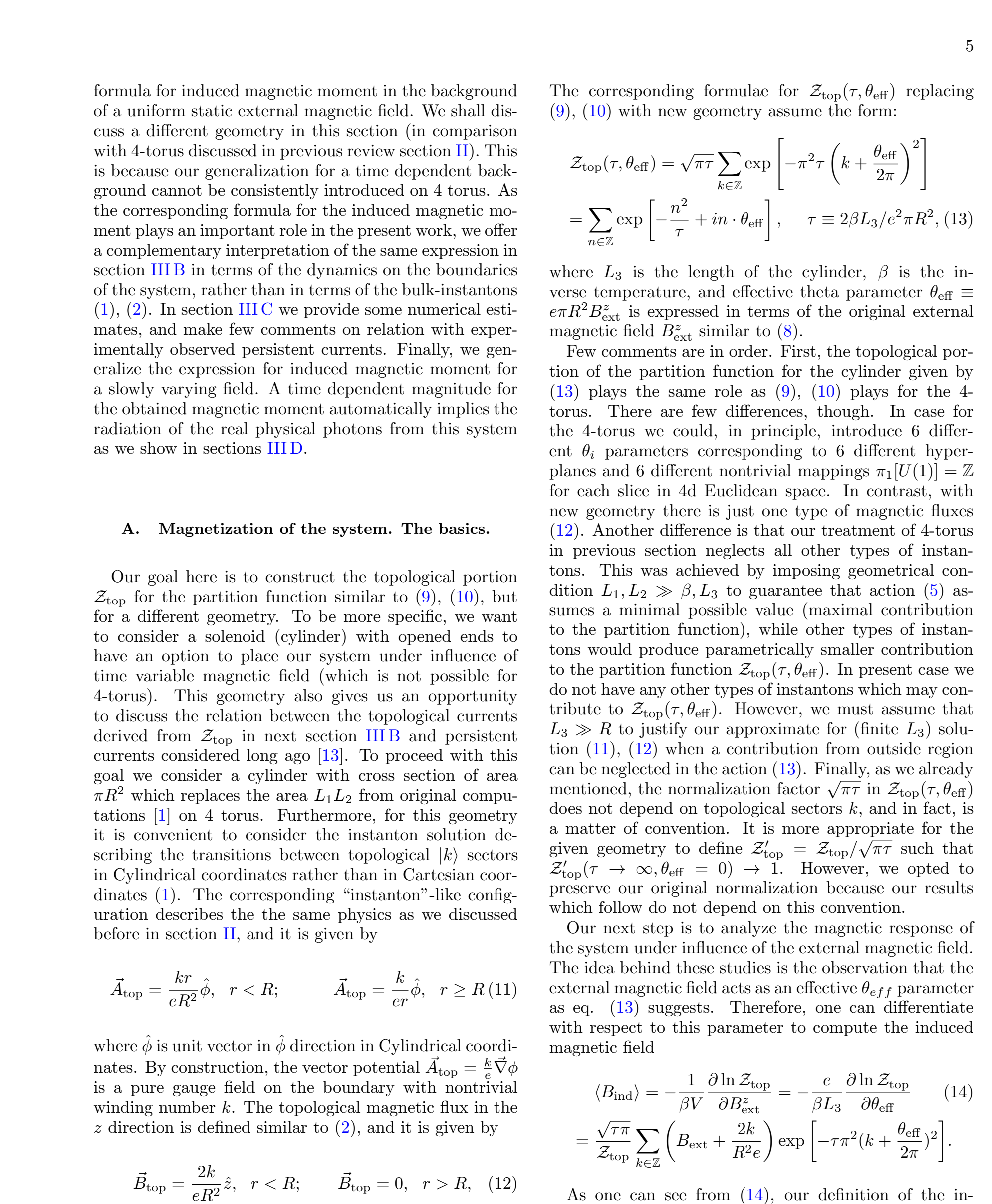

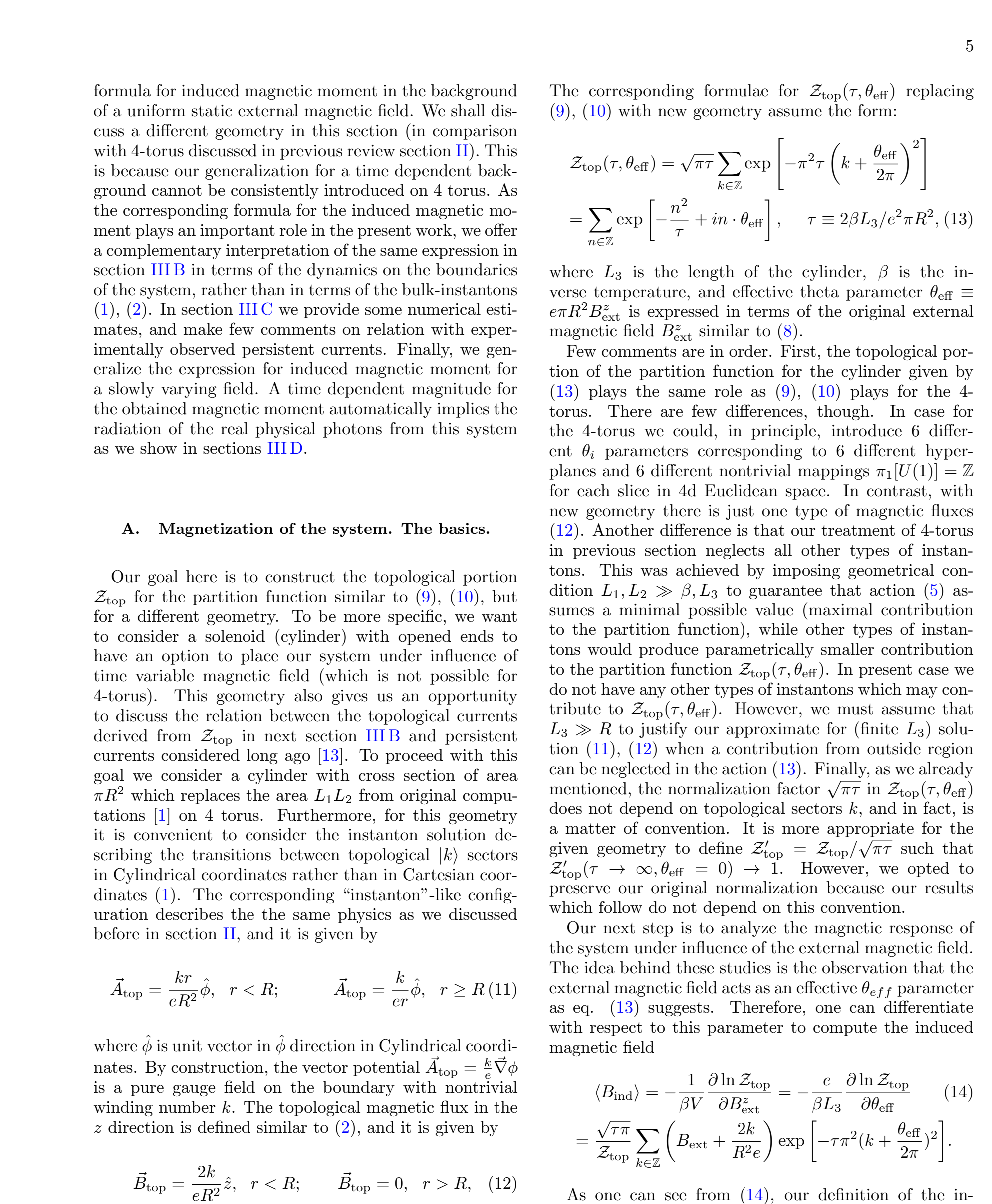

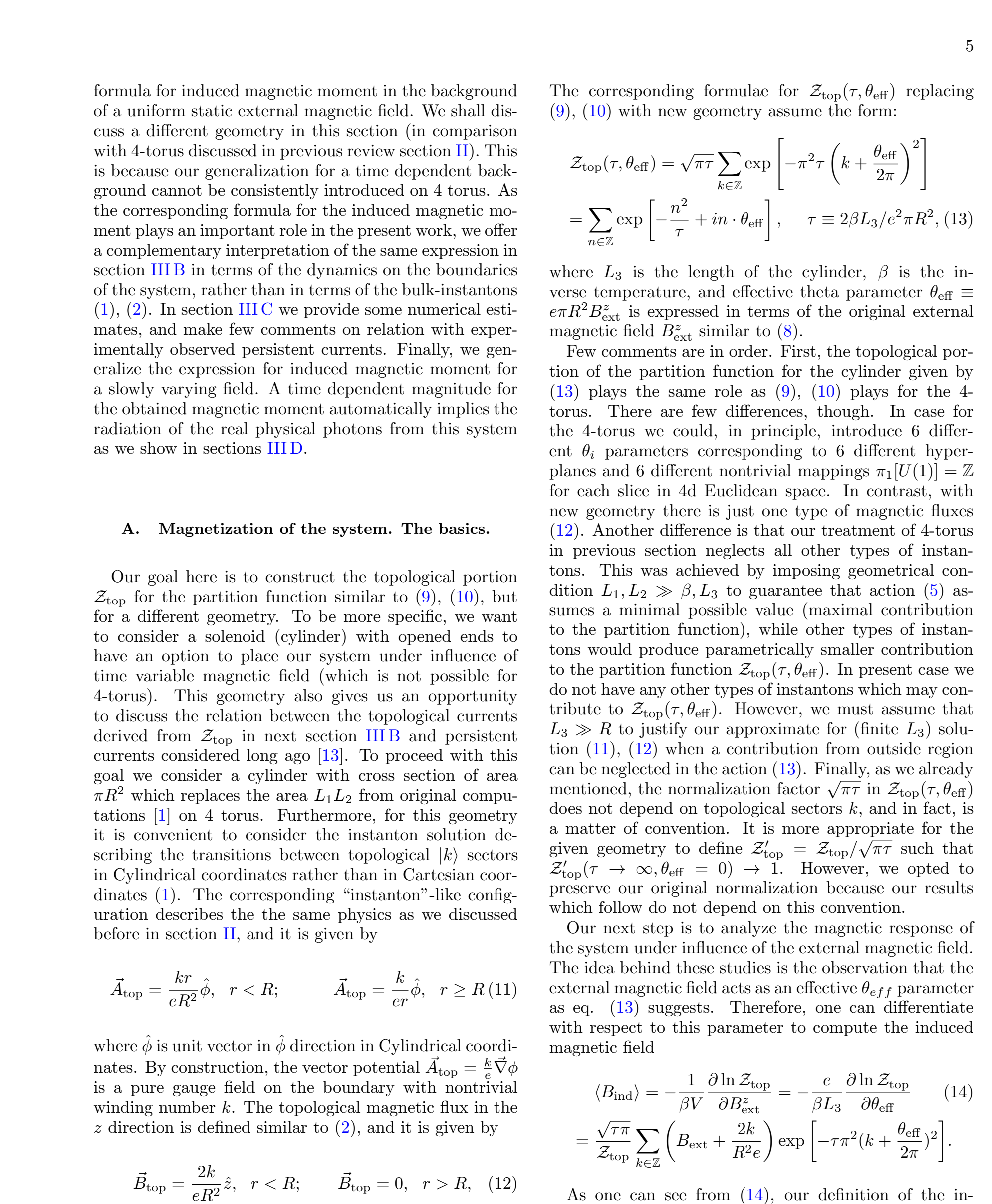

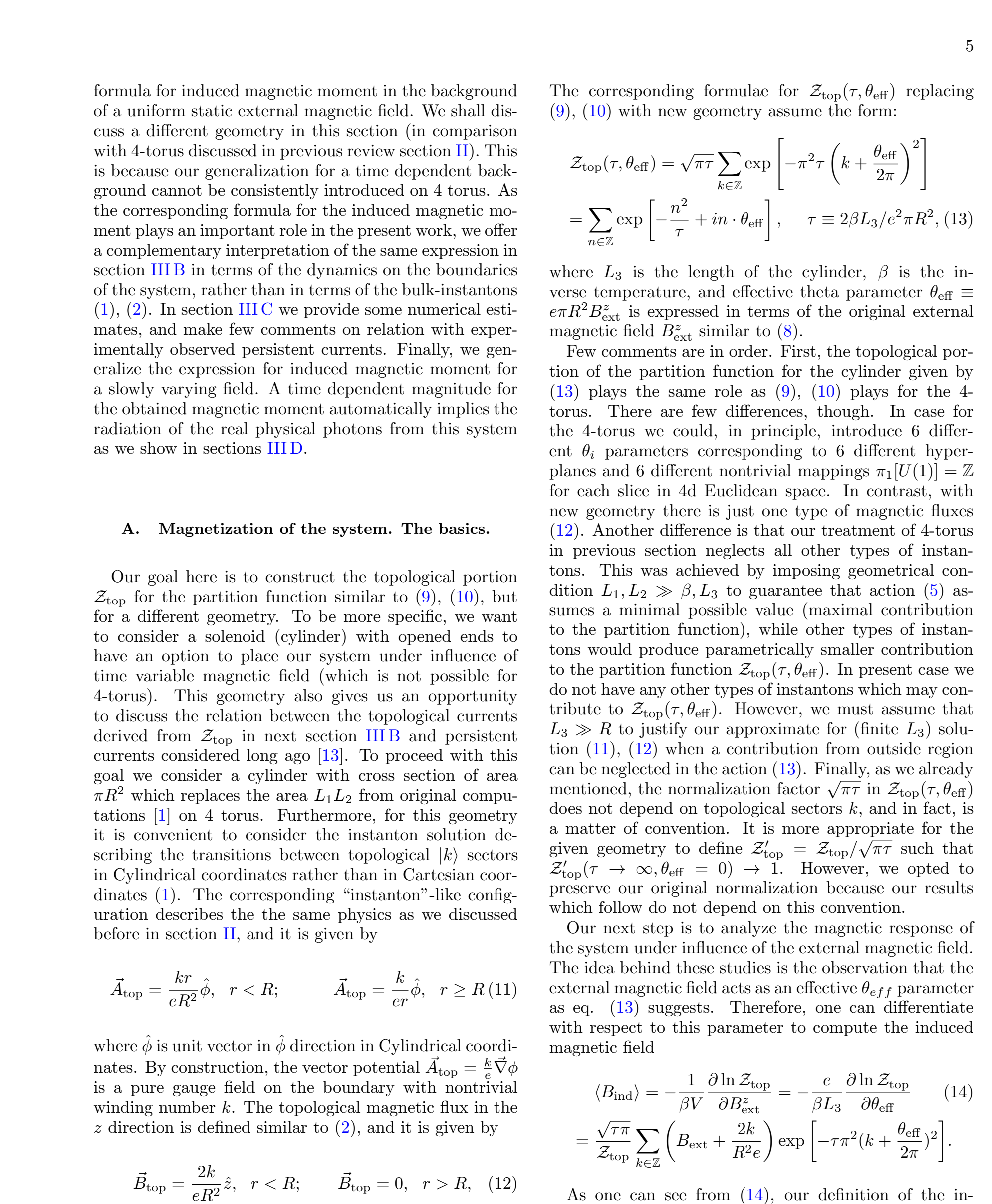

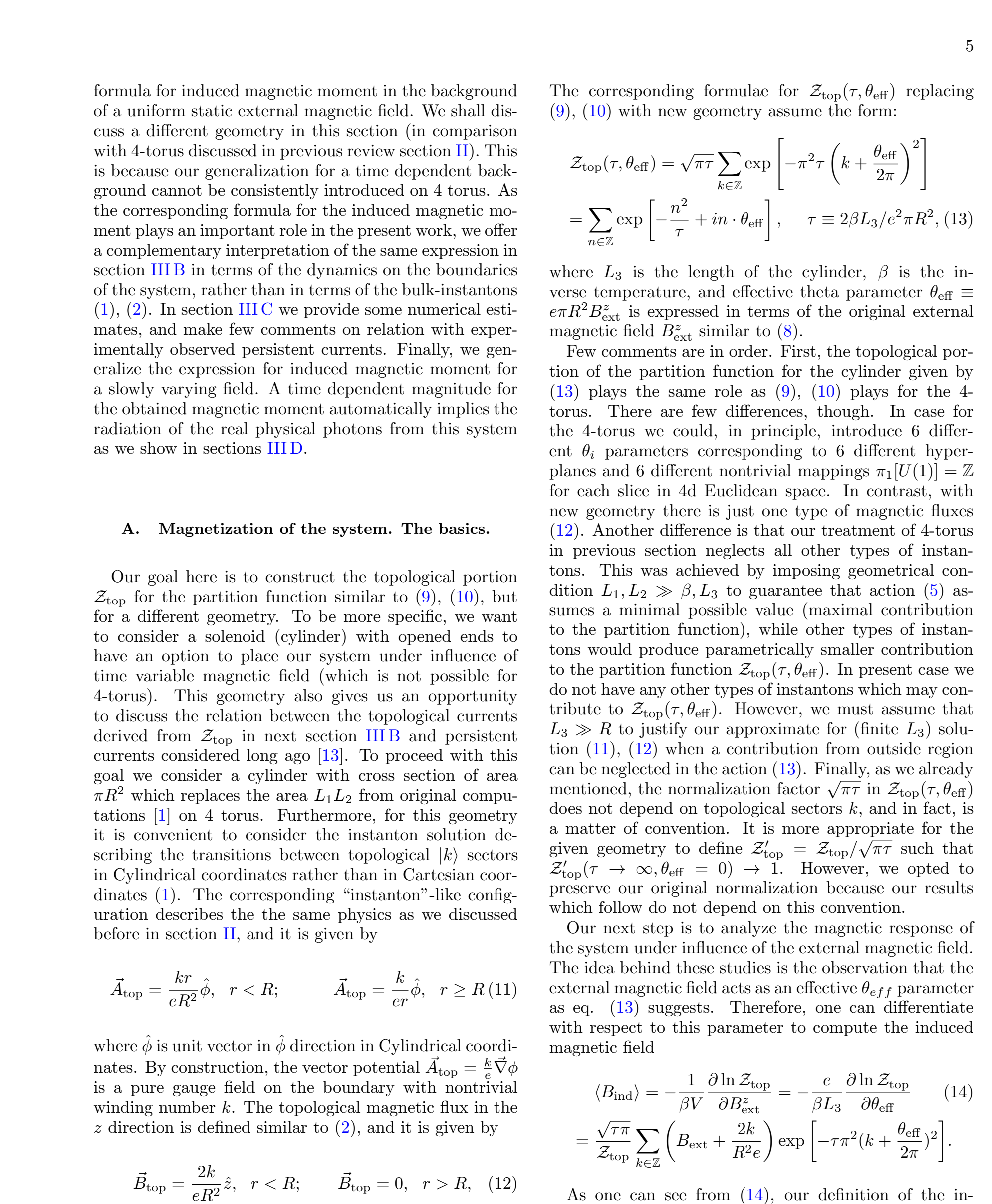

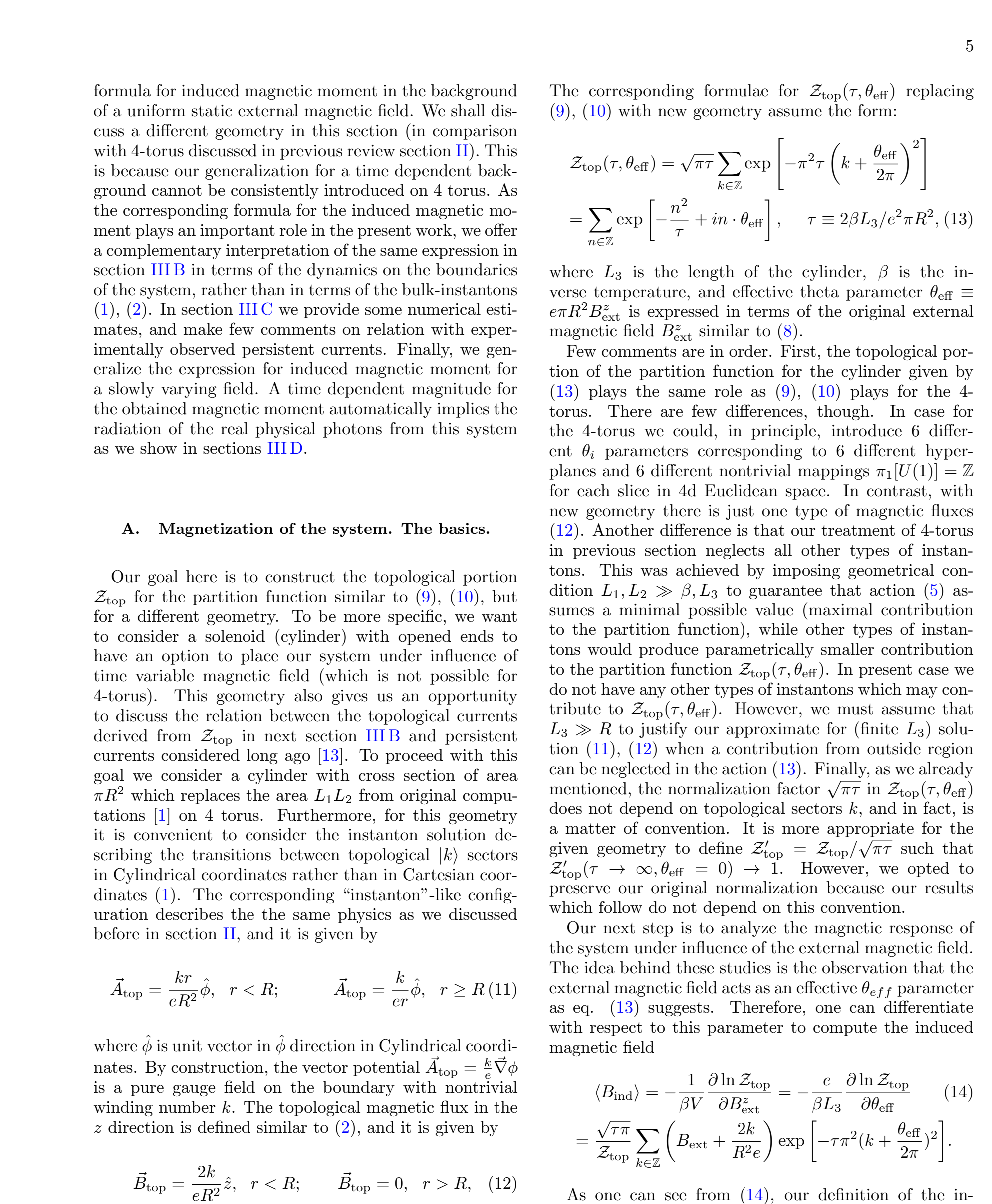

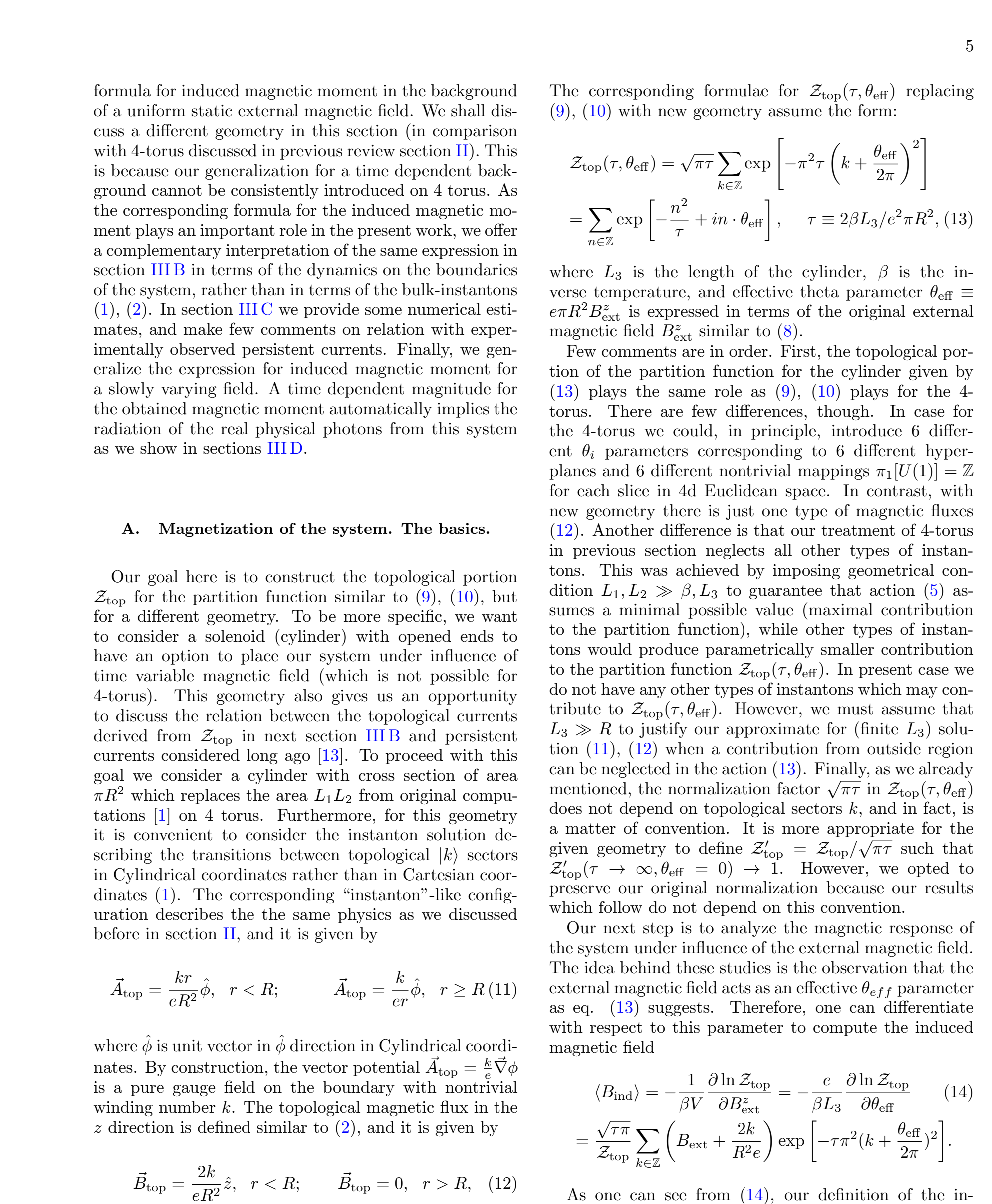

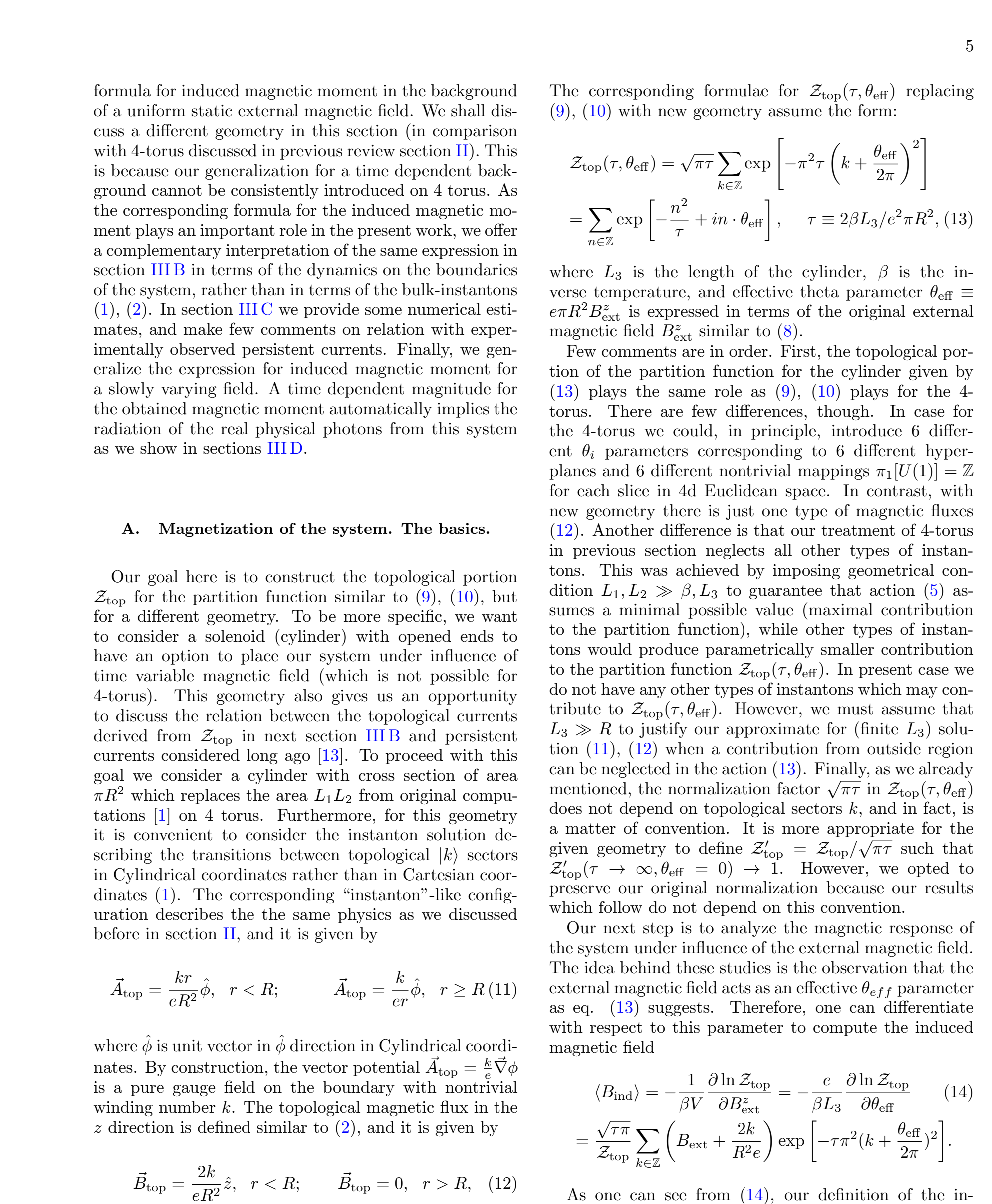

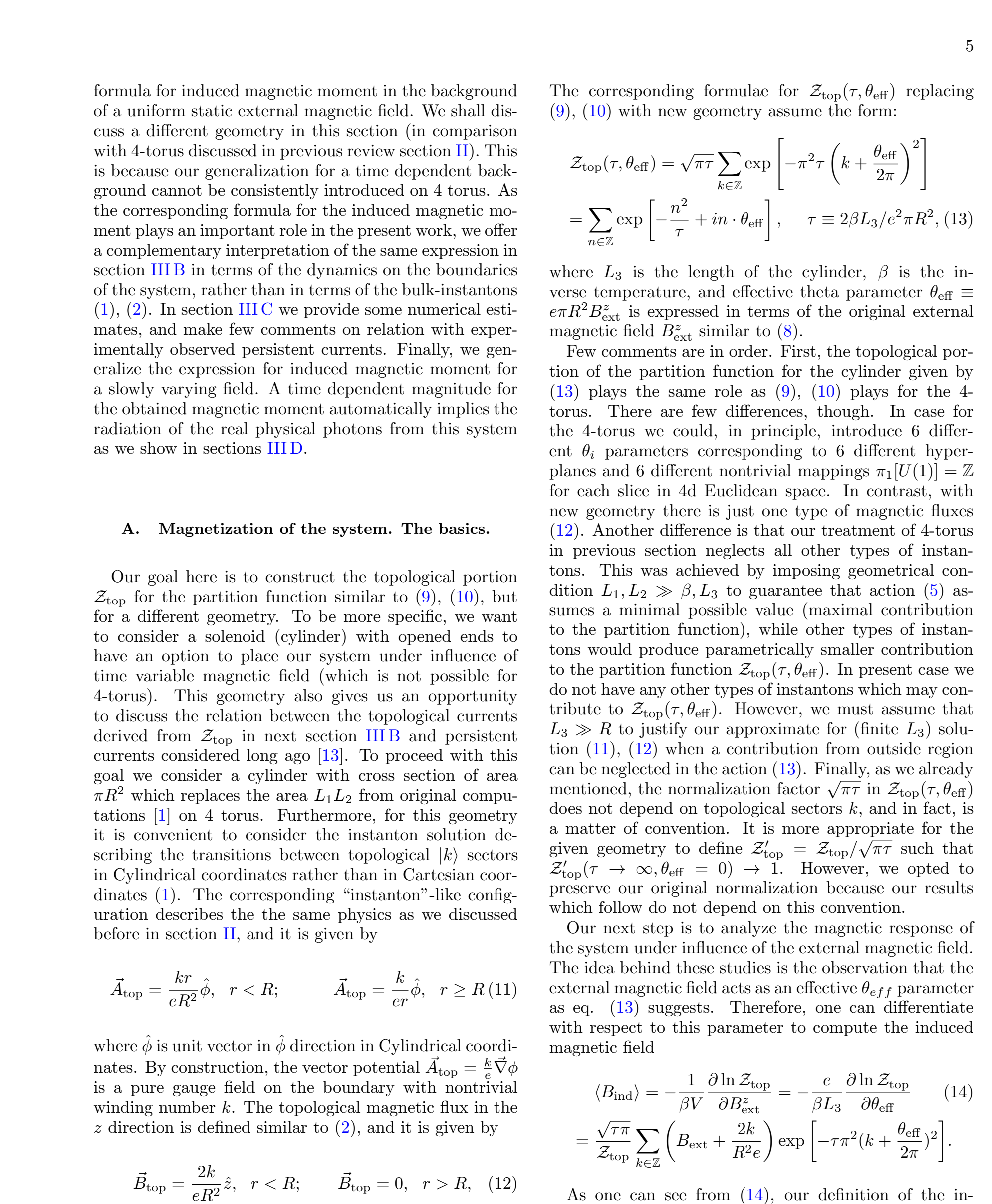

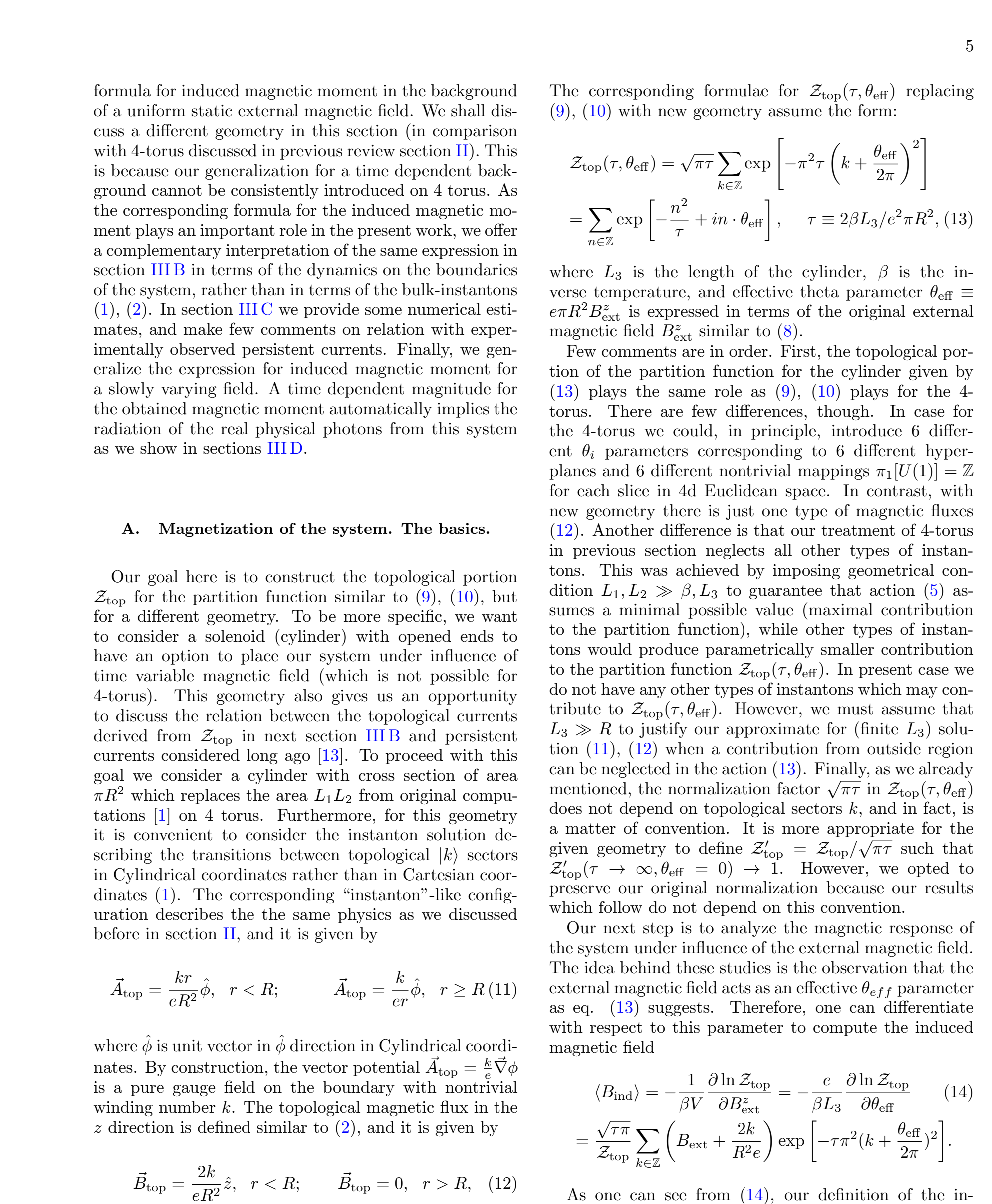

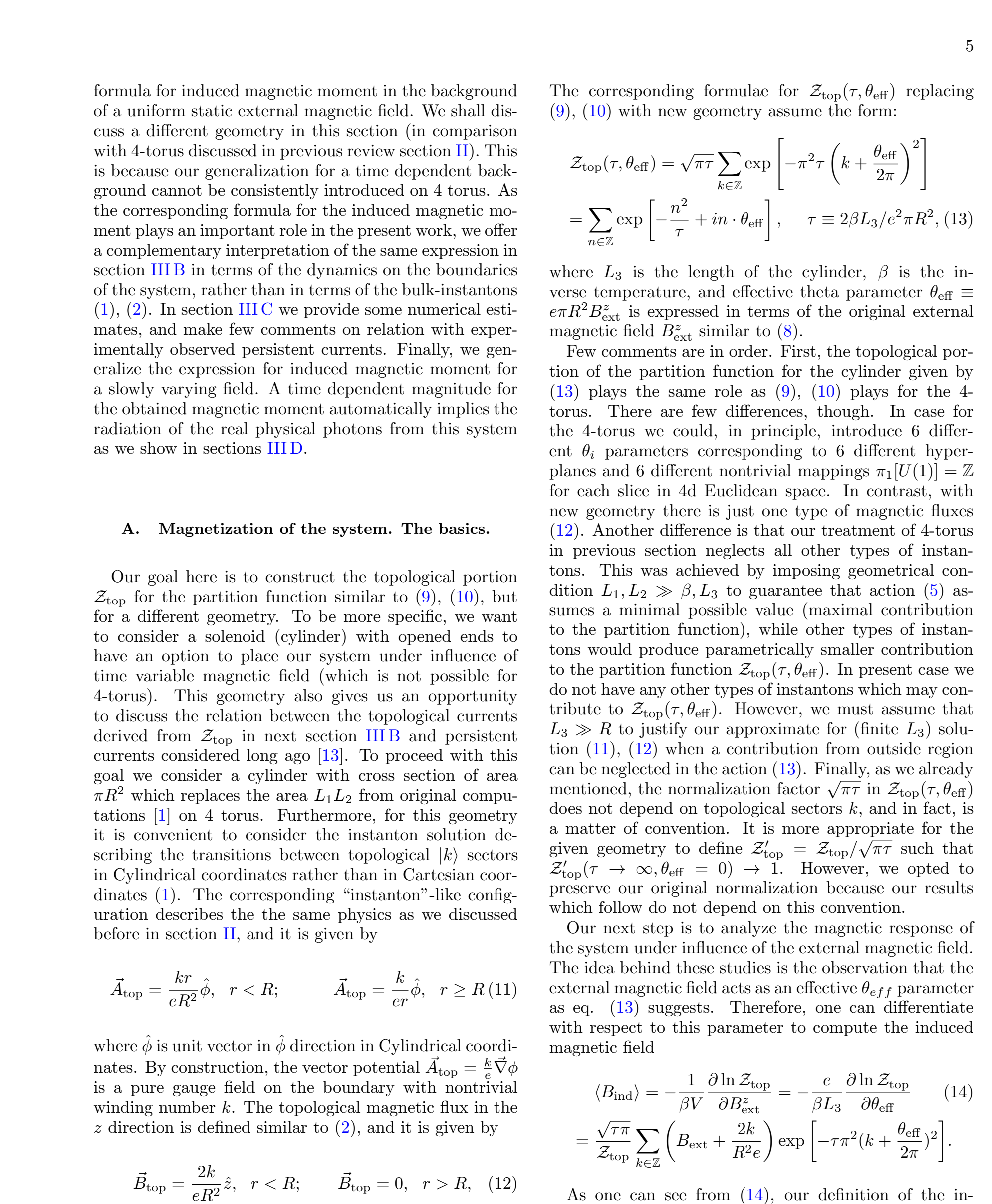

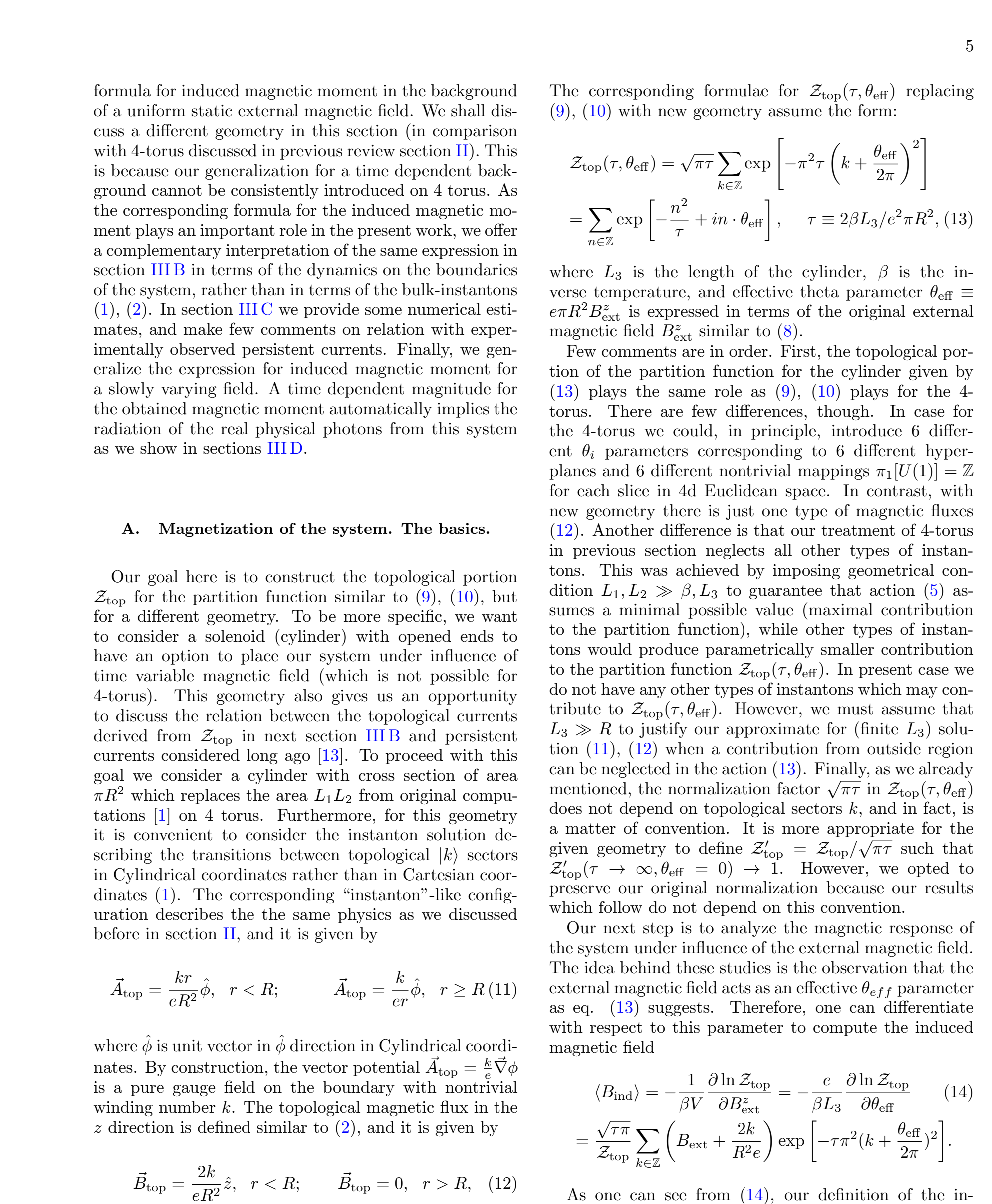

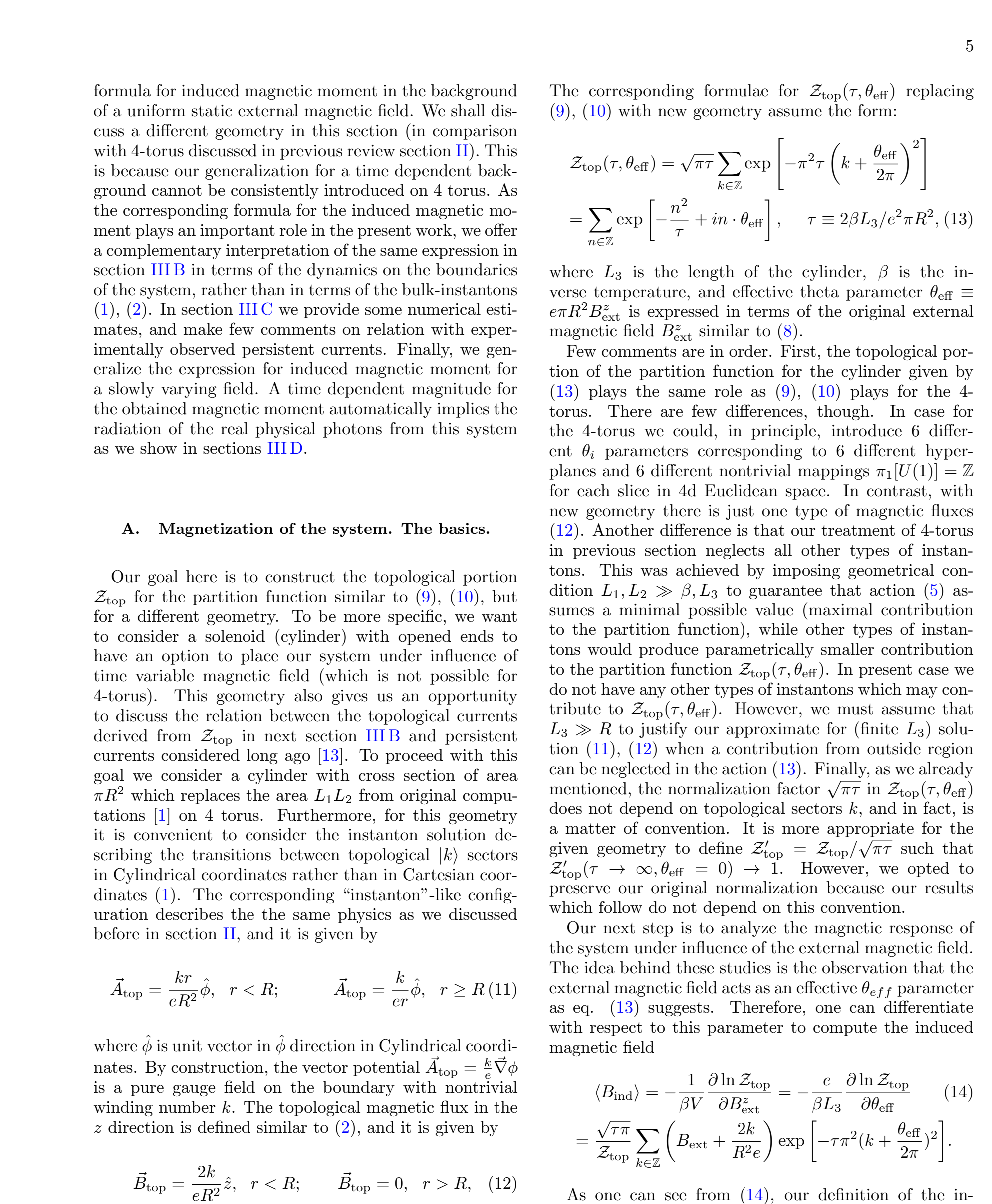

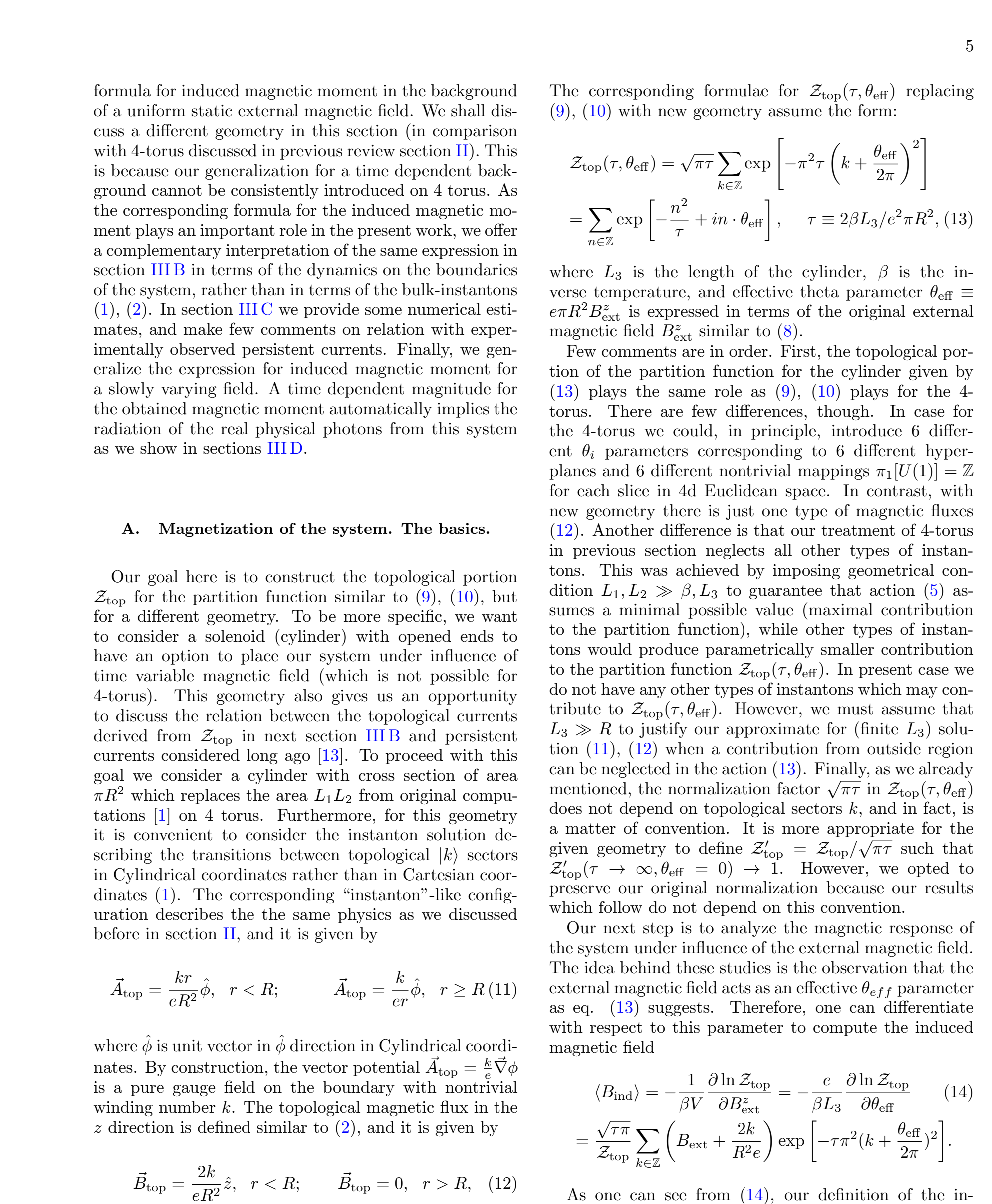

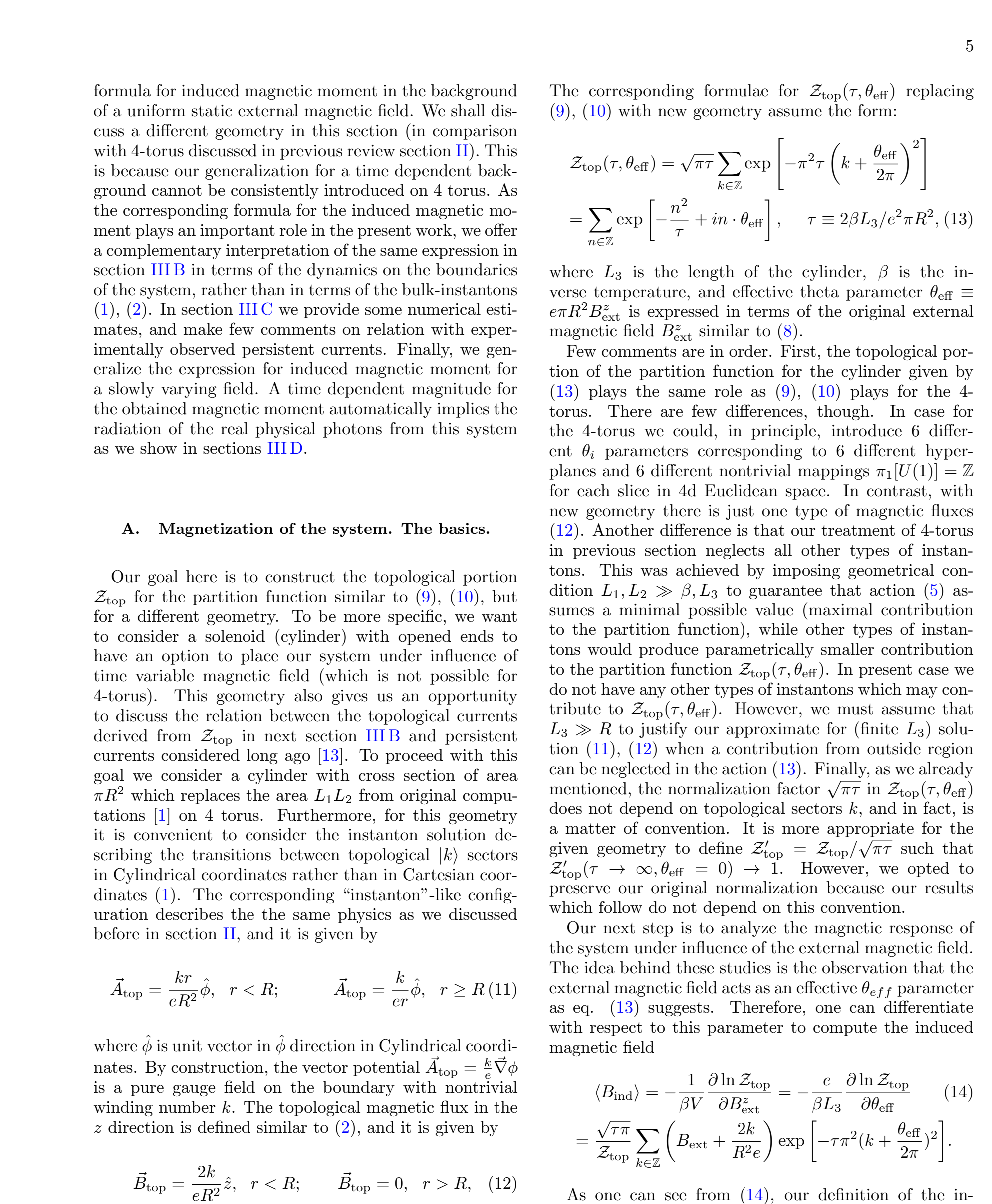

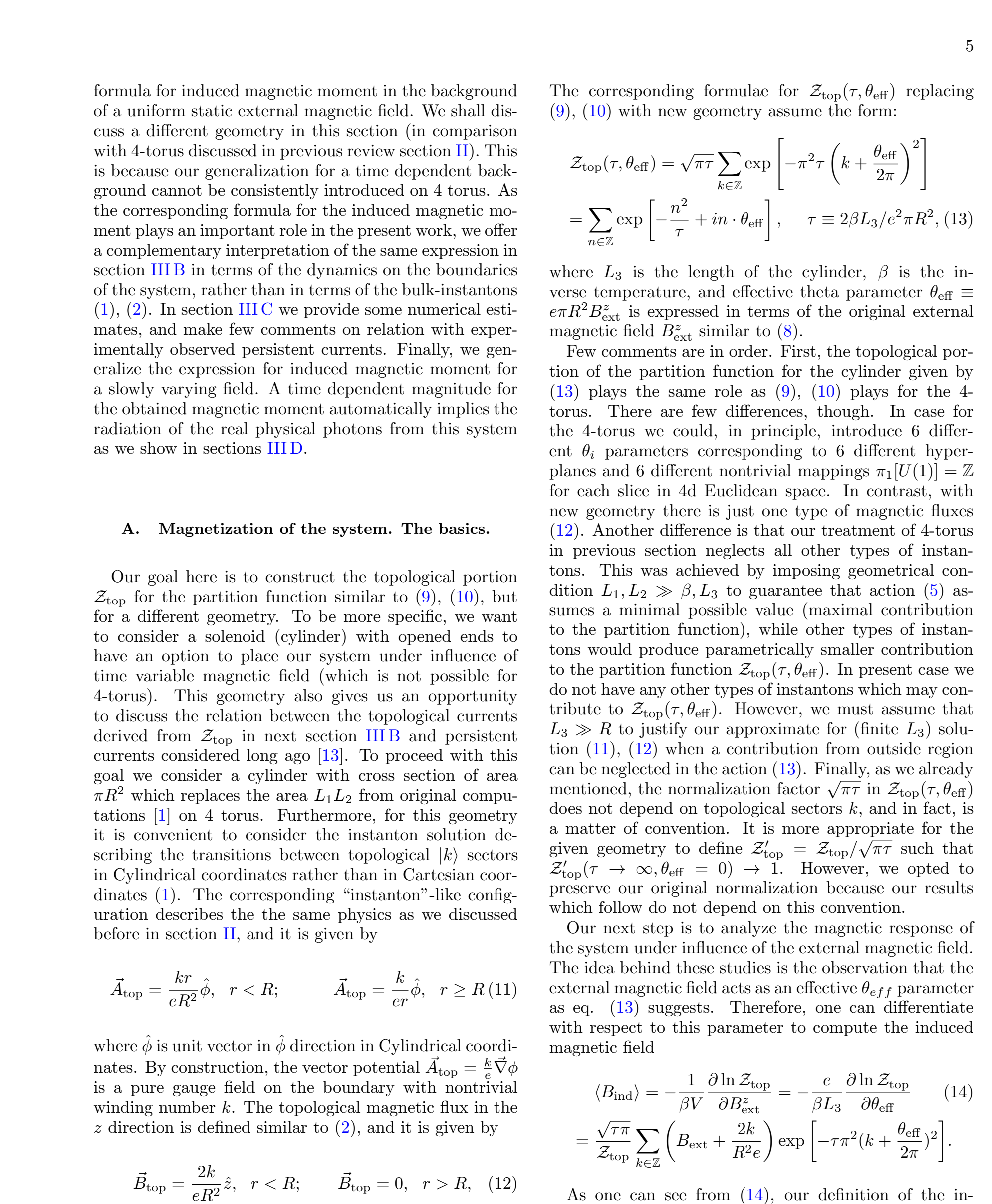

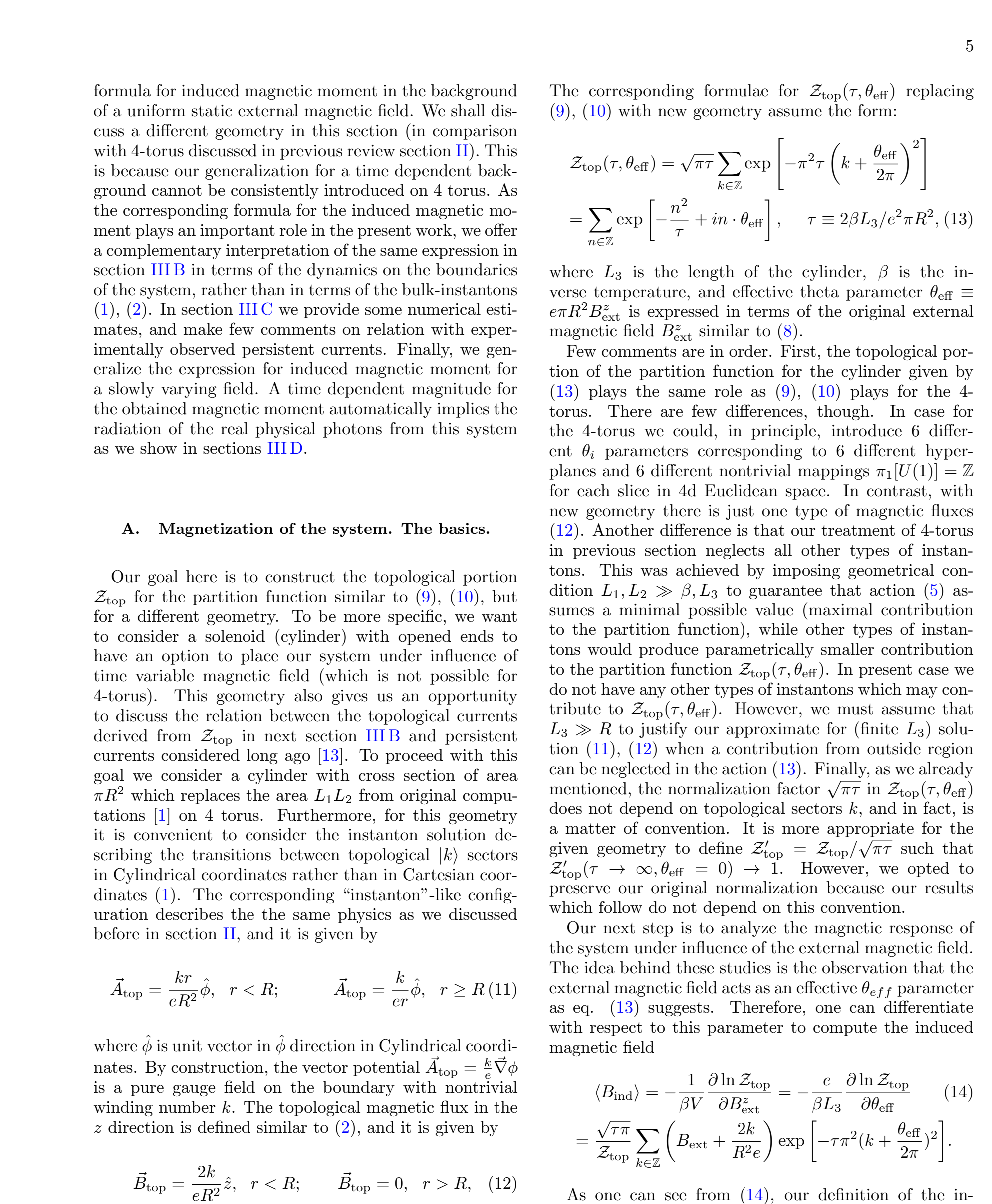

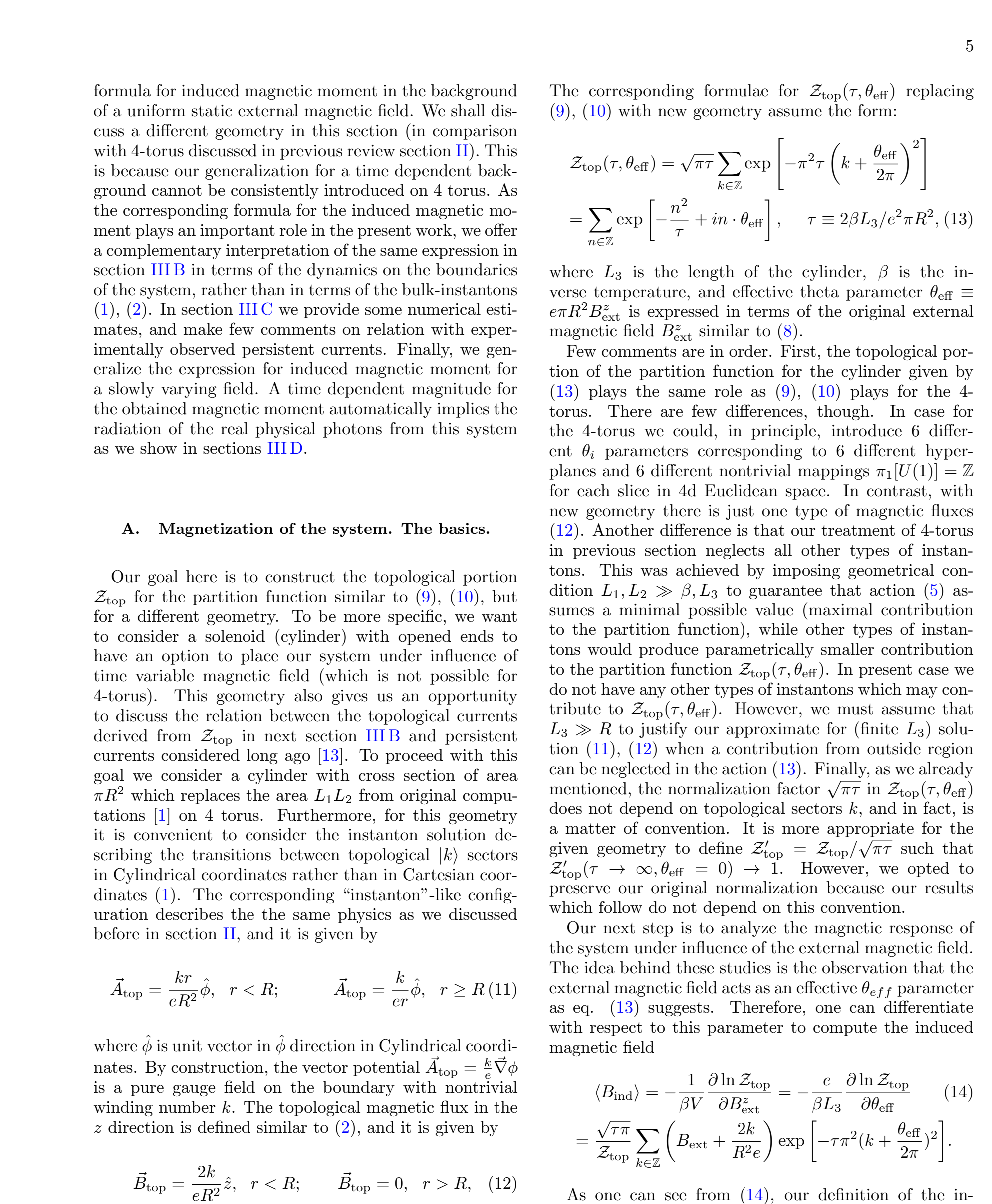

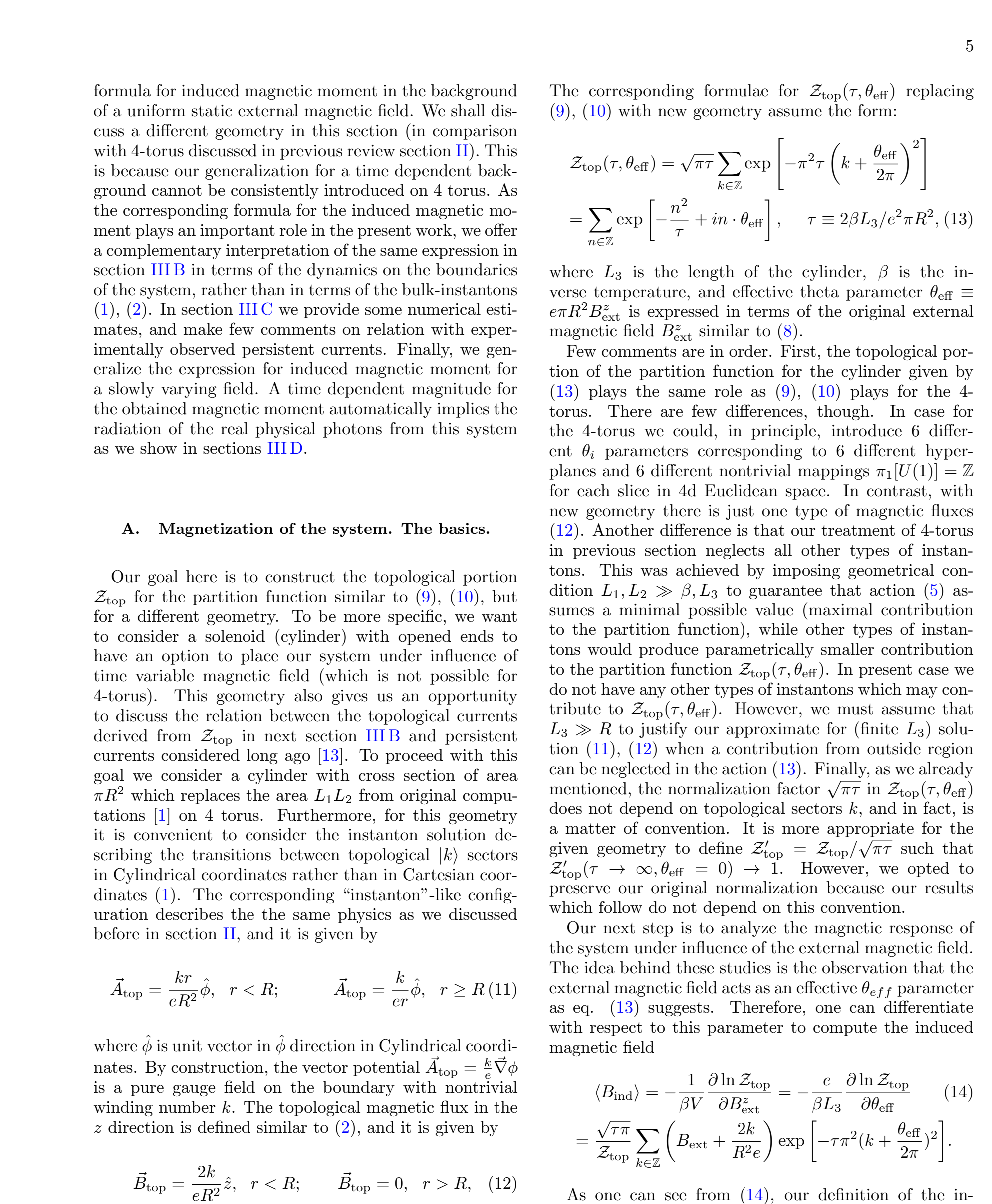

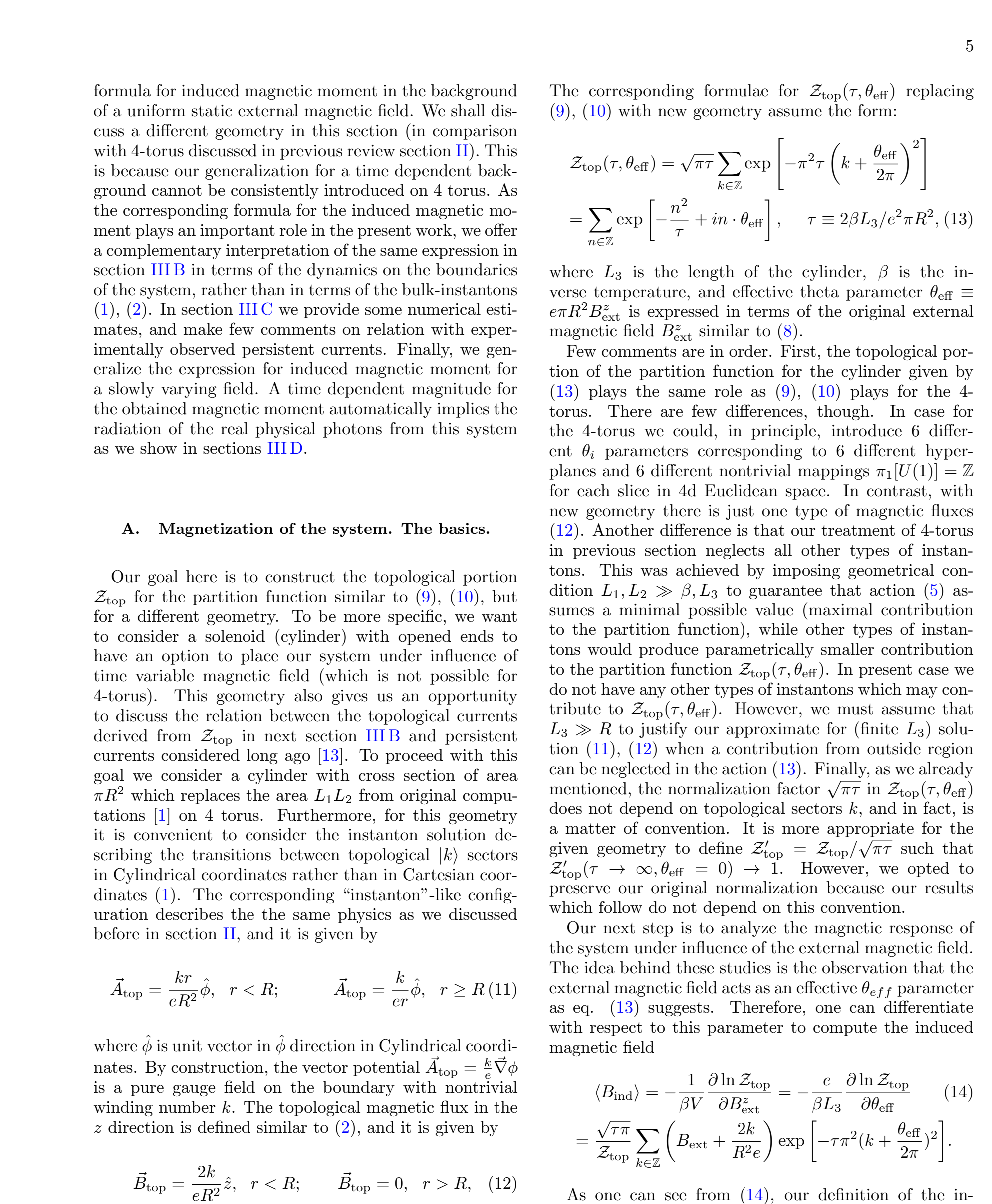

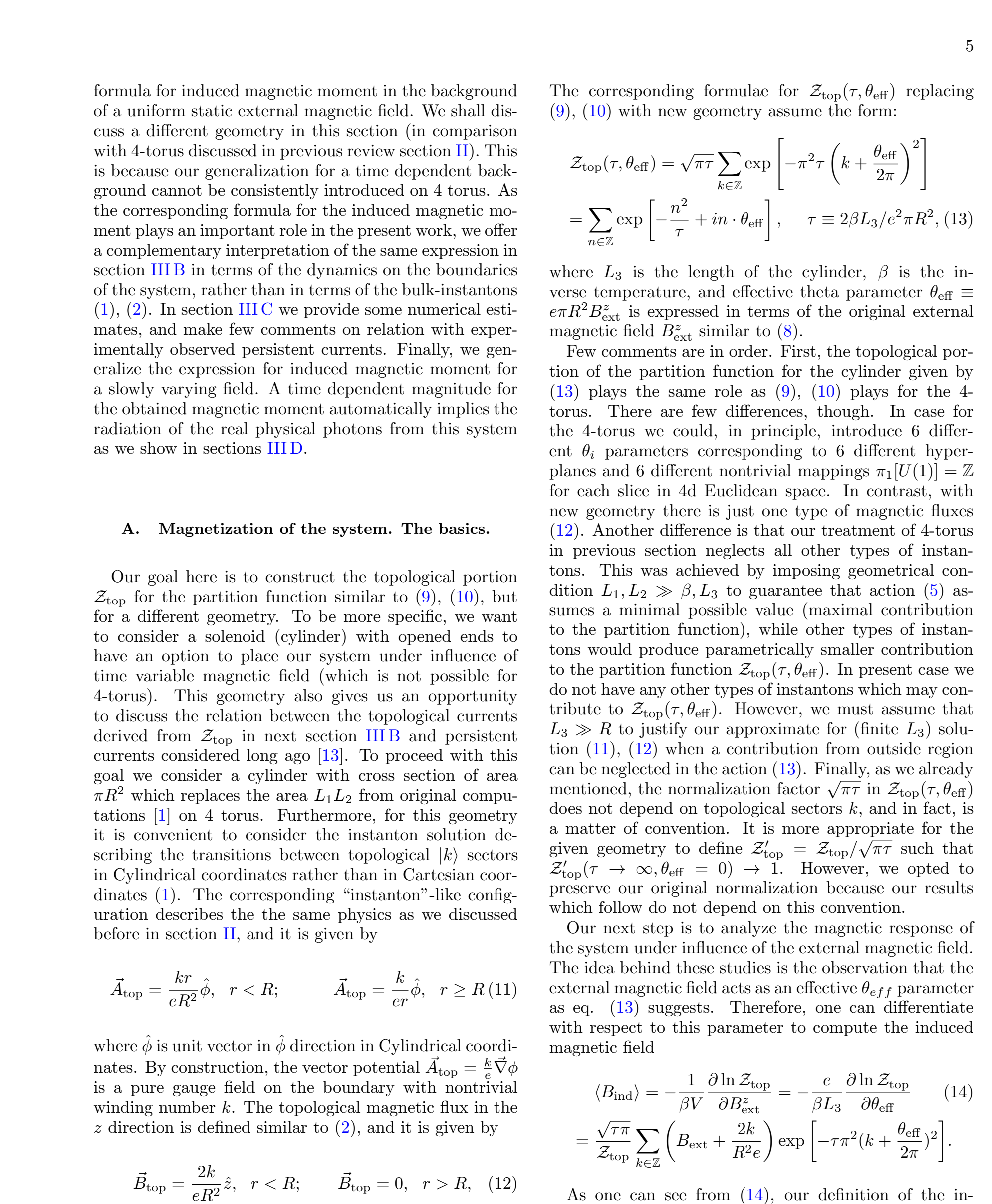

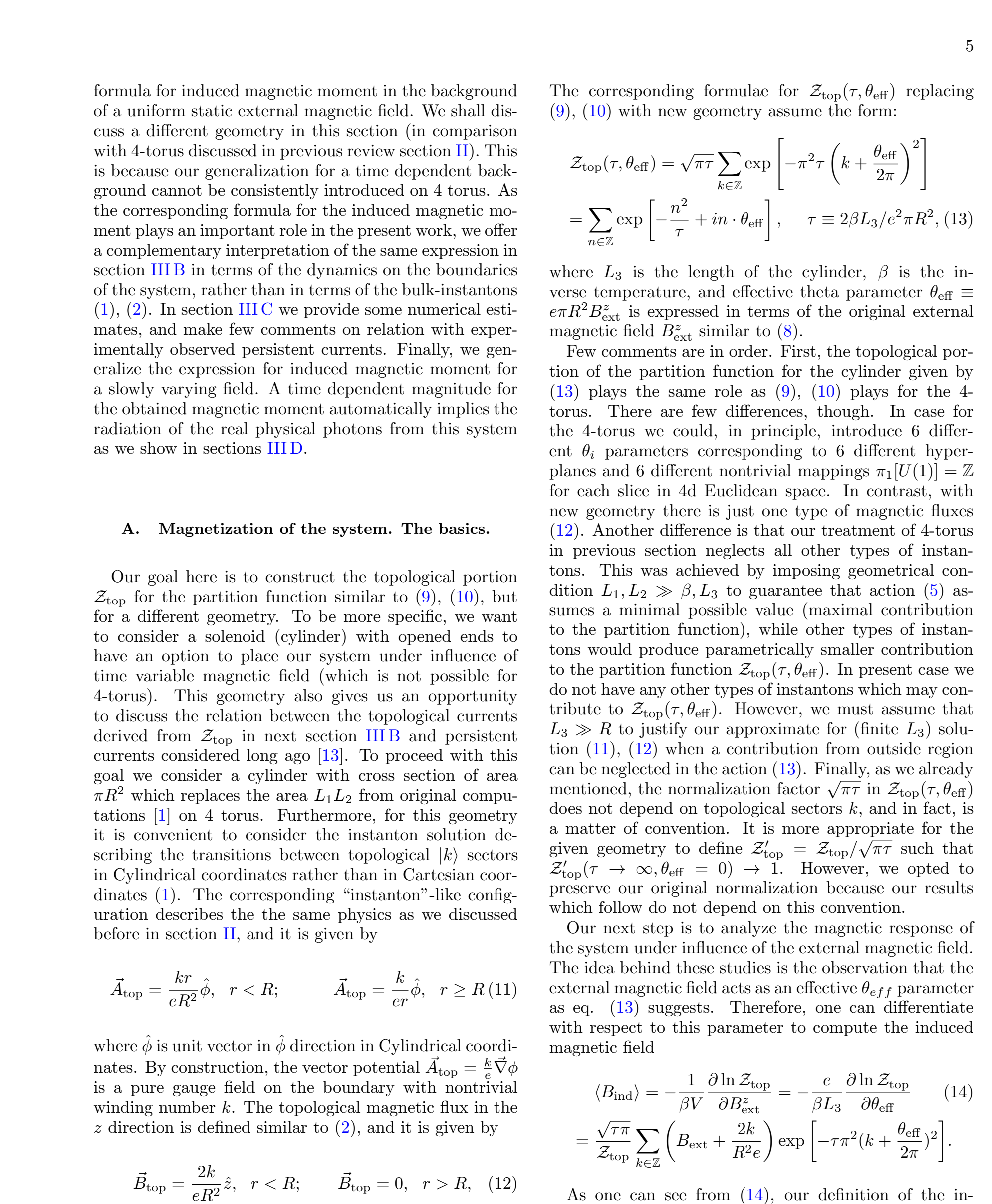

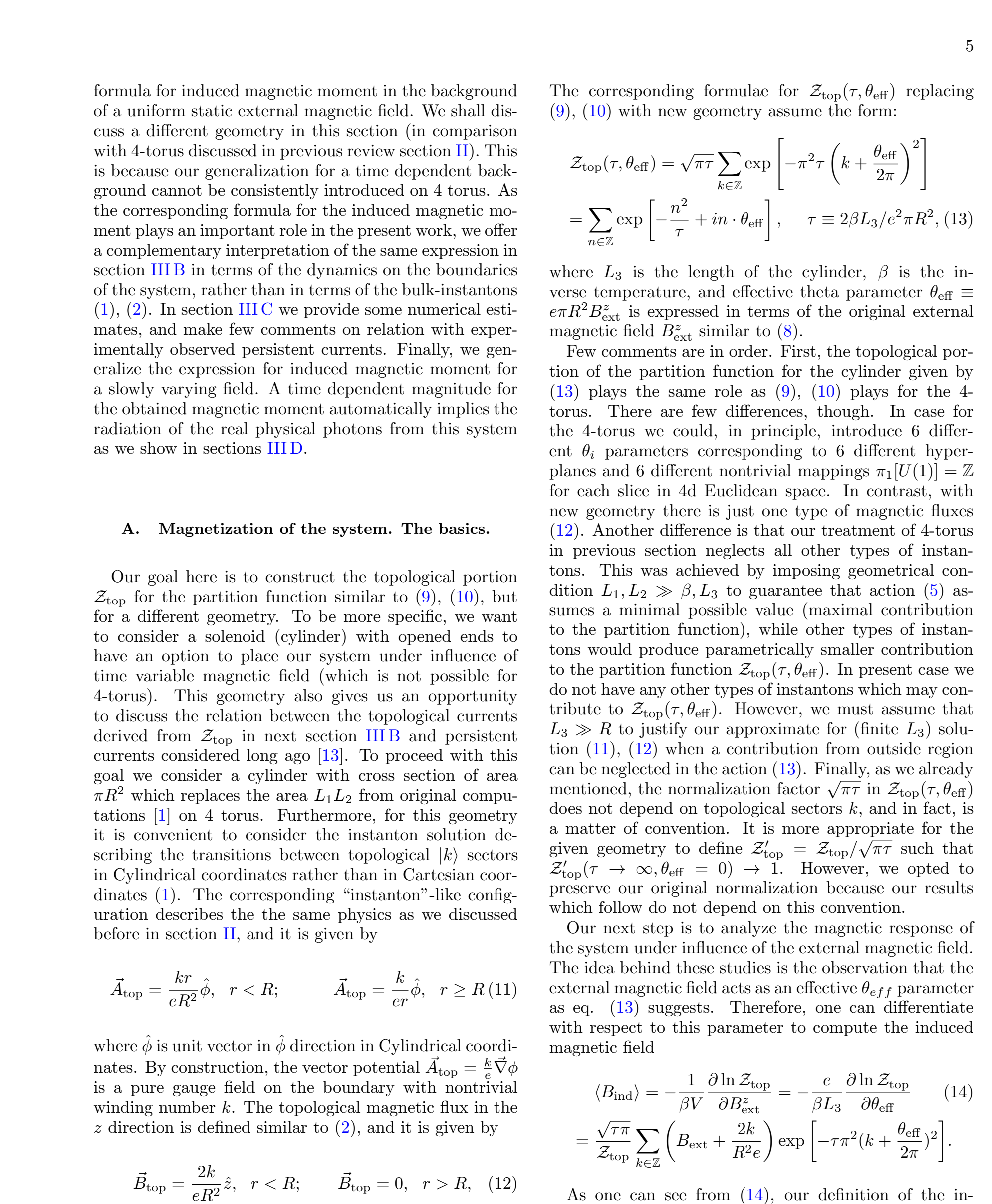

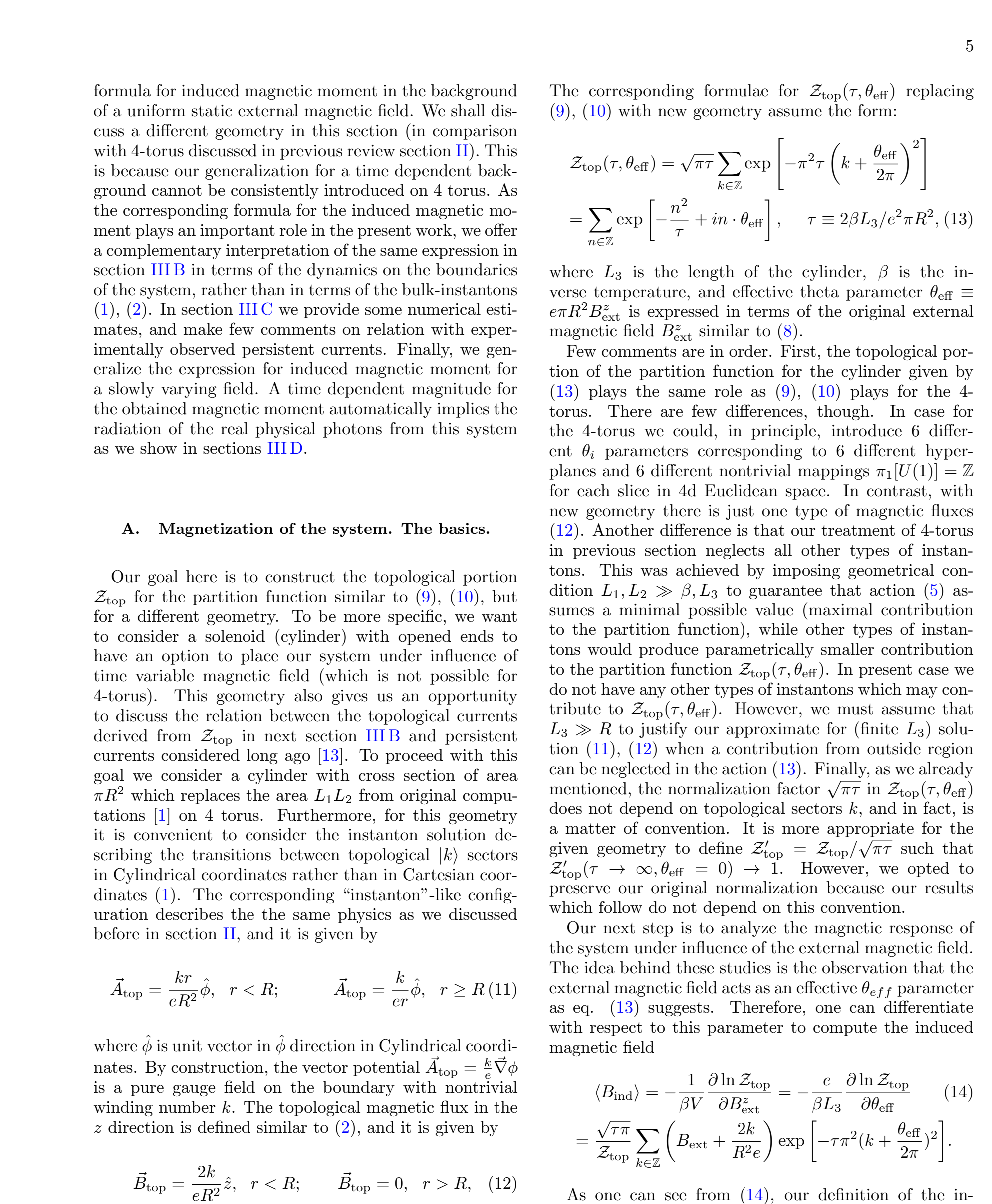

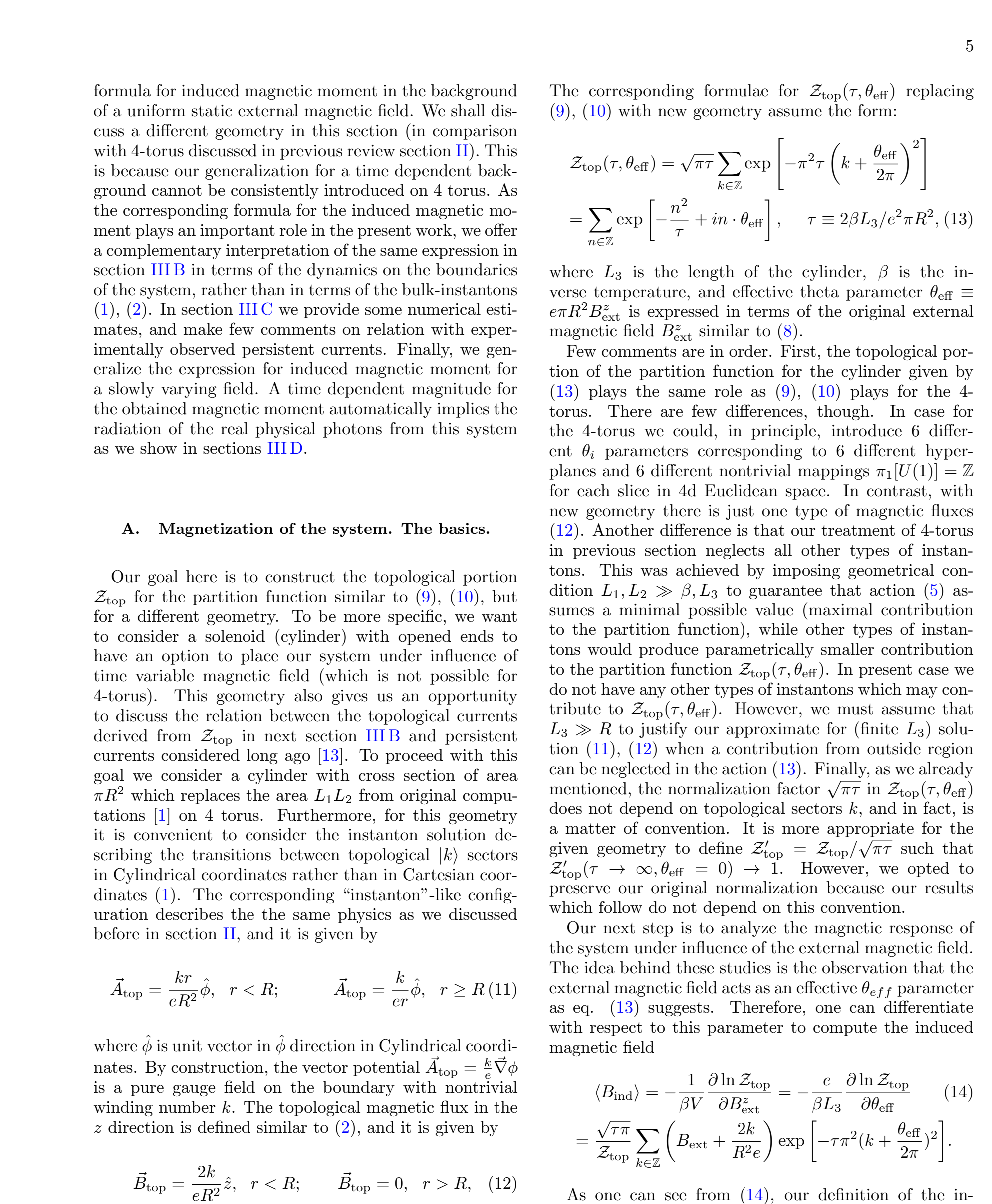

$$
\vec{A}_{\text {top }}=\frac{k r}{e R^{2}} \hat{\phi}, \quad r<R ; \quad \vec{A}_{\text {top }}=\frac{k}{e r} \hat{\phi}, \quad r \geq R(11)
$$

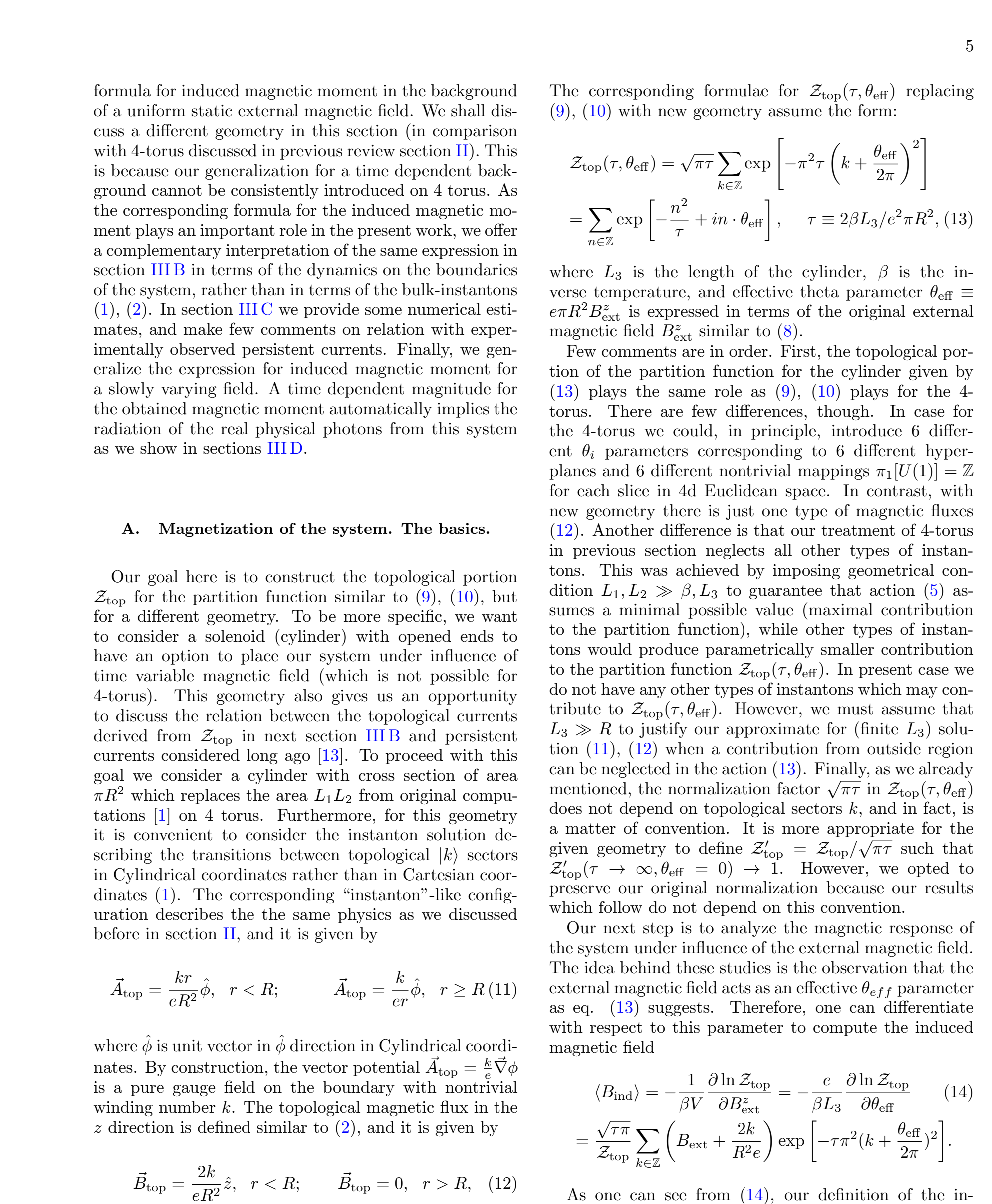

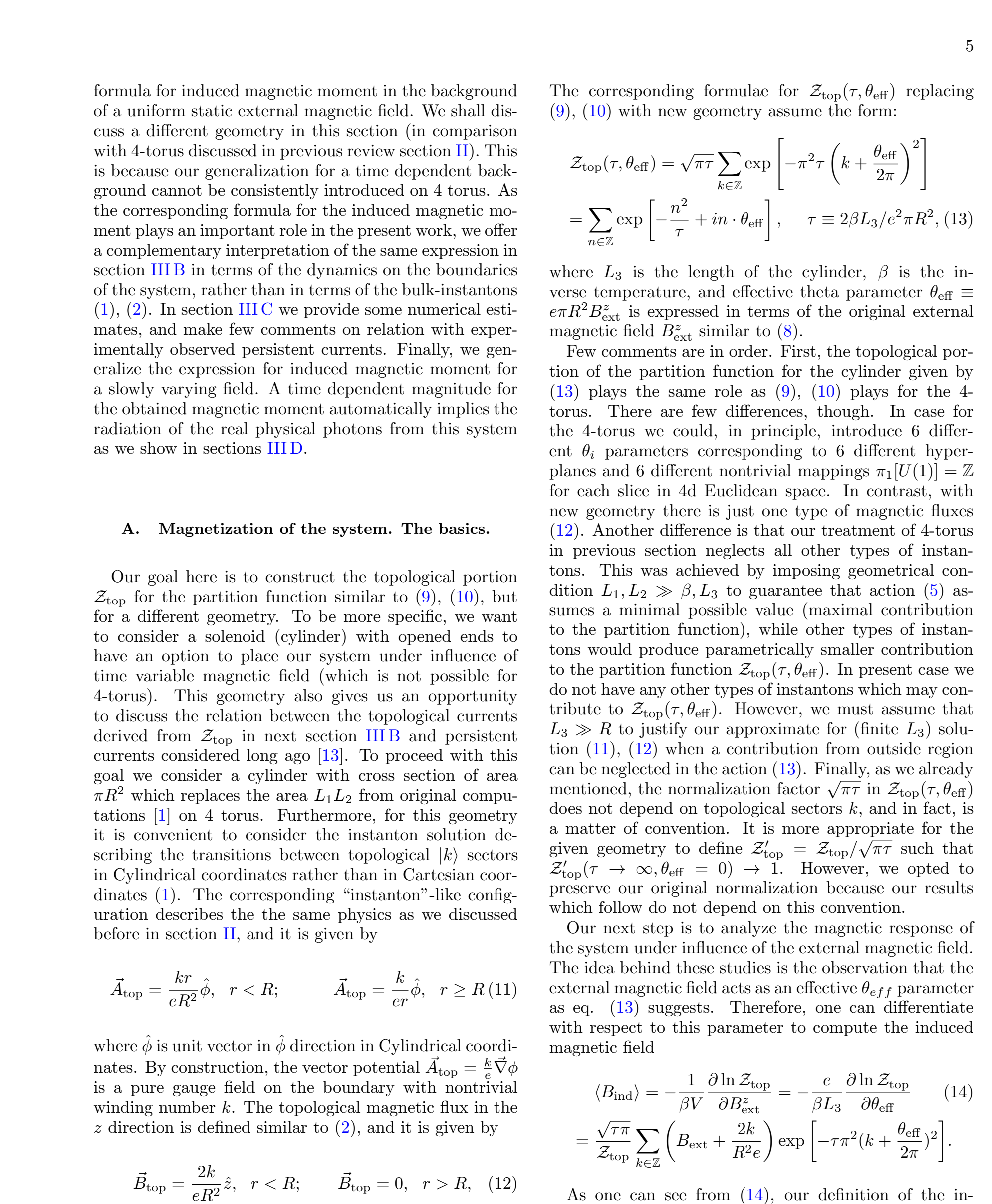

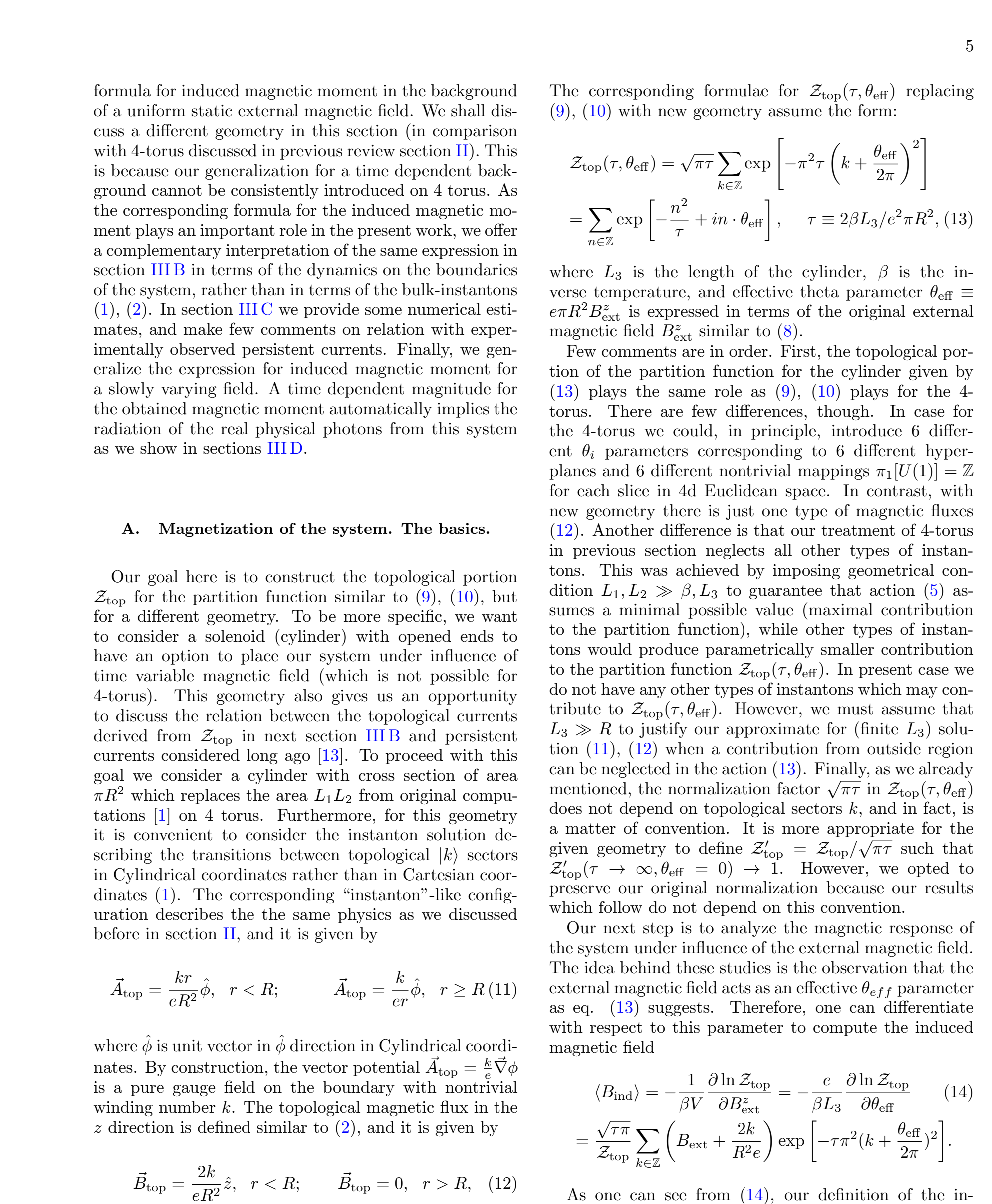

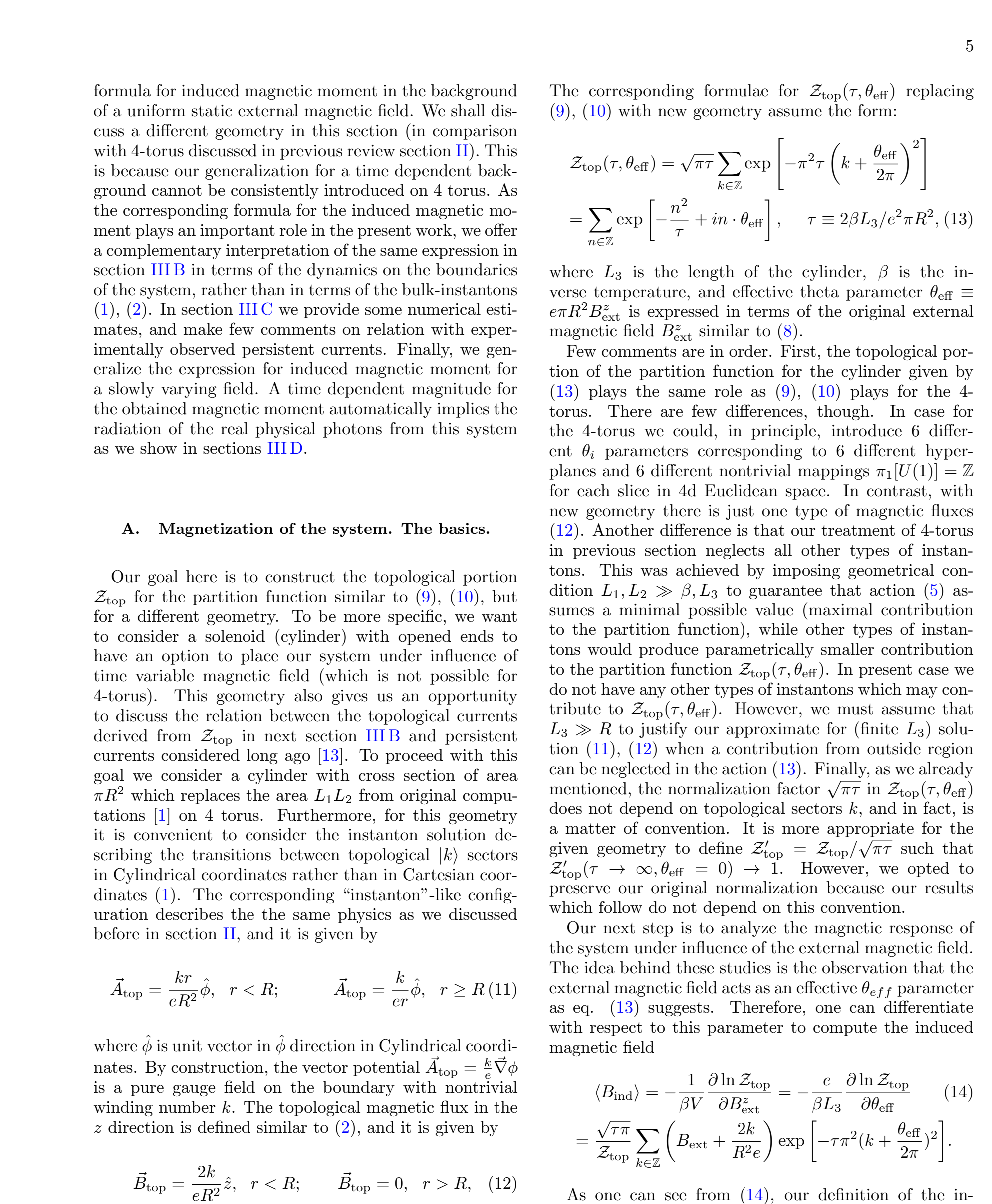

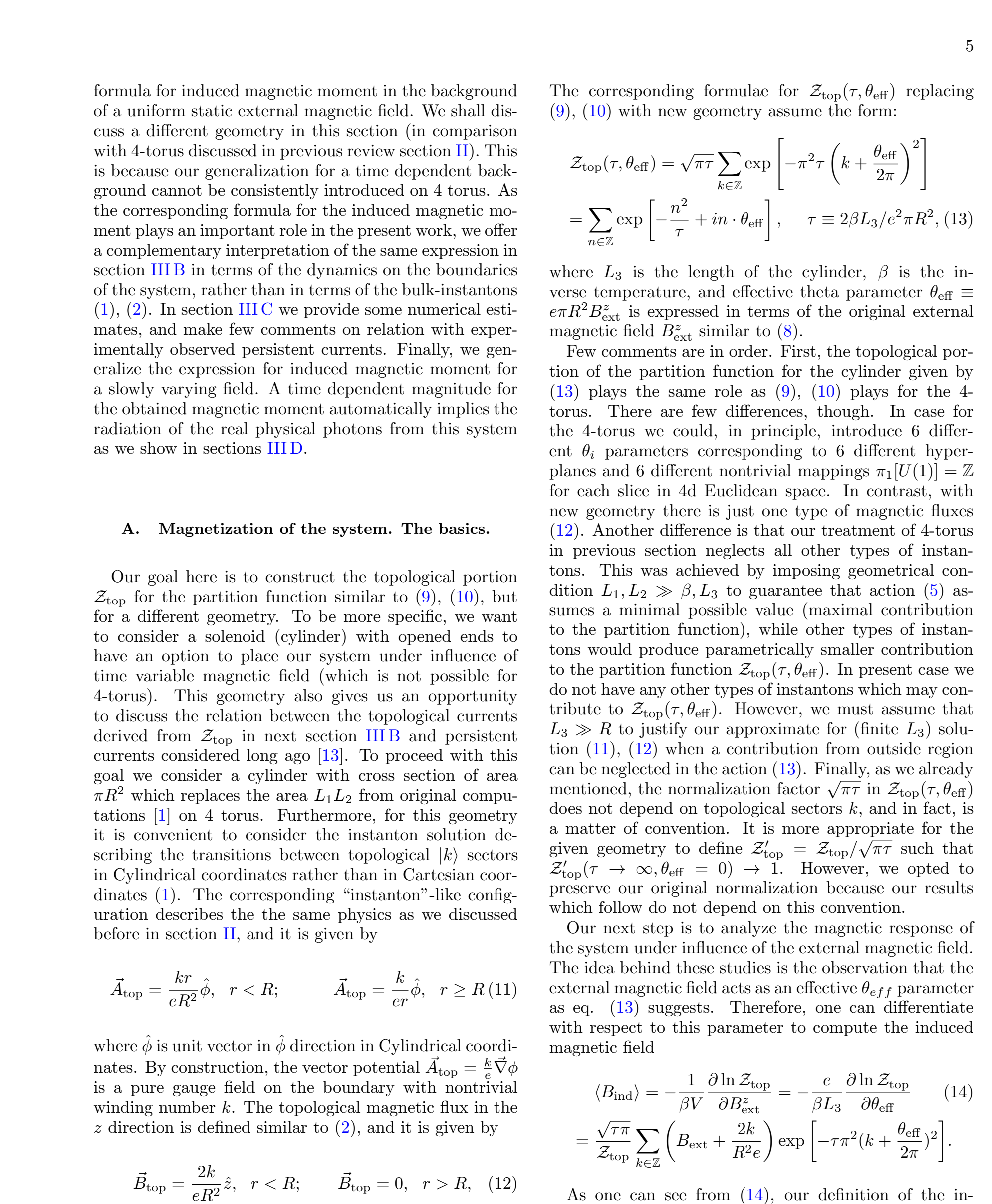

$$
\begin{aligned}
& \vec{B}_{\text {top }}=\frac{2 k}{e R^{2}} \hat{z}, \quad r<R ; \quad \vec{B}_{\text {top }}=0, \quad r>R, \\
& \Phi=e \int d x_{1} d x_{2} B_{\text {top }}^{z}=e \oint_{r=R} \vec{A}_{\text {top }} \cdot d \vec{l}=2 \pi k .
\end{aligned}
$$

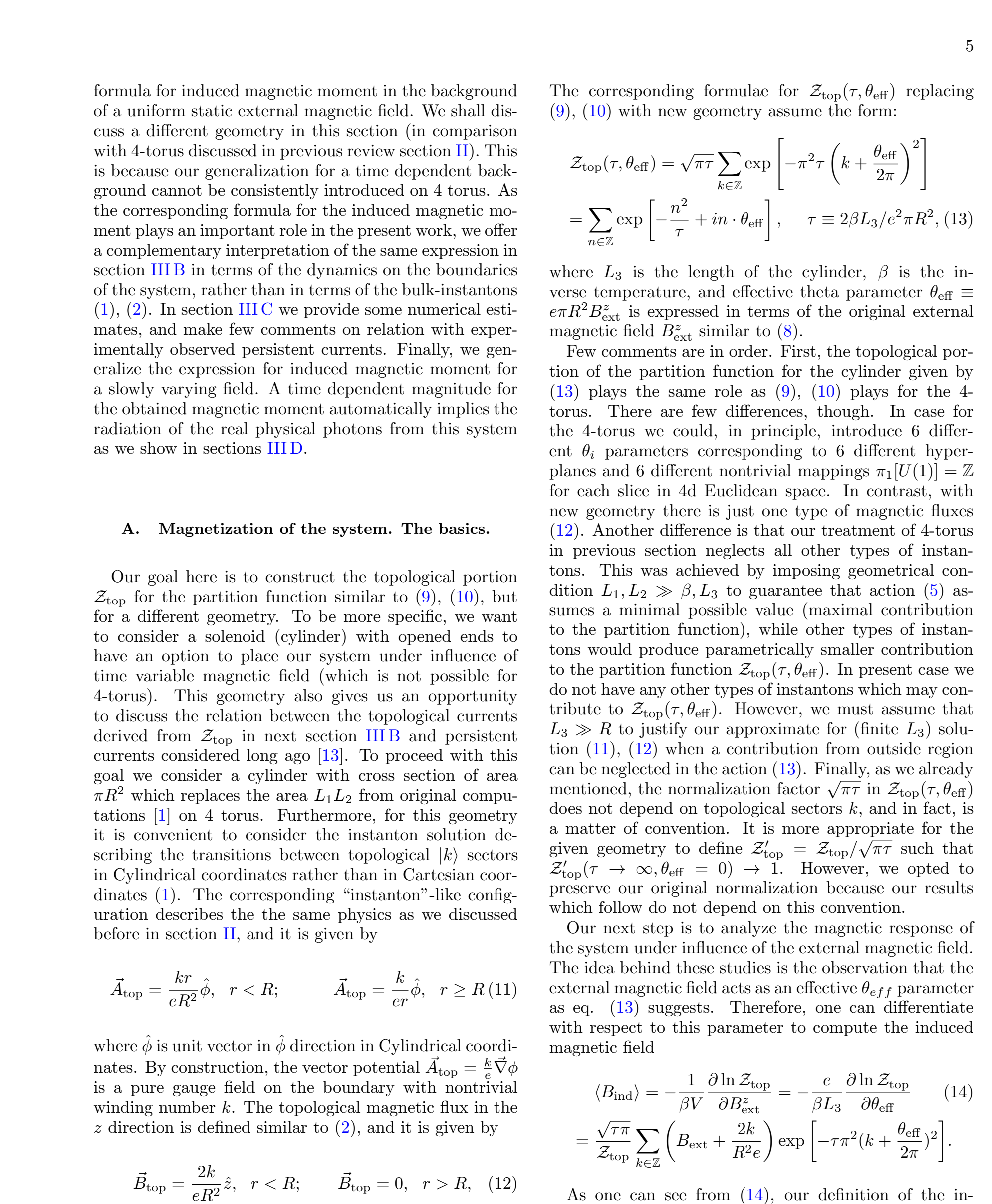
duced field accounts for the total field which includes both terms: the external part as well as the topological

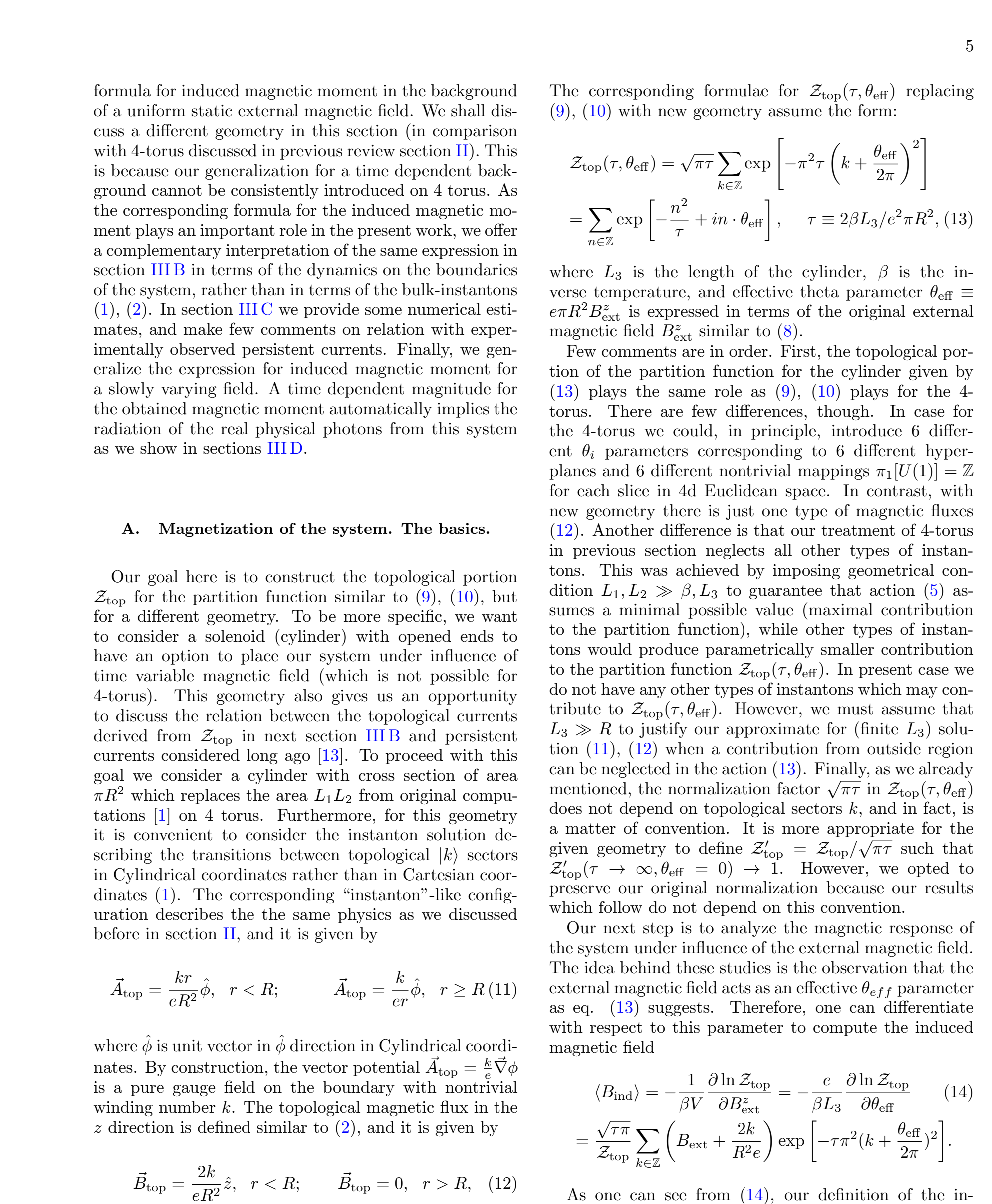

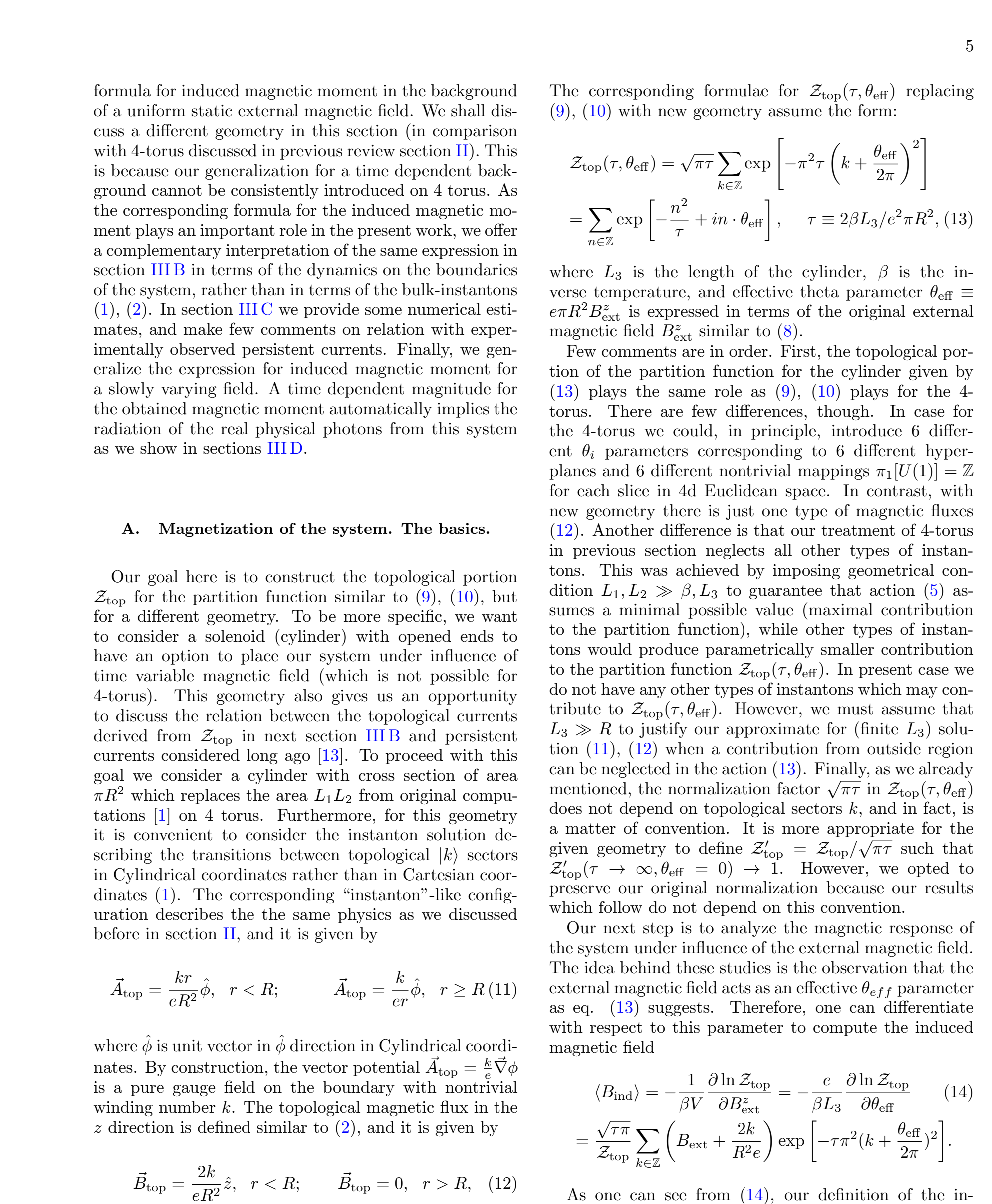

$$
\begin{aligned}
& \mathcal{Z}_{\text {top }}\left(\tau, \theta_{\text {eff }}\right)=\sqrt{\pi \tau} \sum_{k \in \mathbb{Z}} \exp \left[-\pi^{2} \tau\left(k+\frac{\theta_{\text {eff }}}{2 \pi}\right)^{2}\right] \\
& =\sum_{n \in \mathbb{Z}} \exp \left[-\frac{n^{2}}{\tau}+i n \cdot \theta_{\text {eff }}\right], \quad \tau \equiv 2 \beta L_{3} / e^{2} \pi R^{2}
\end{aligned}
$$

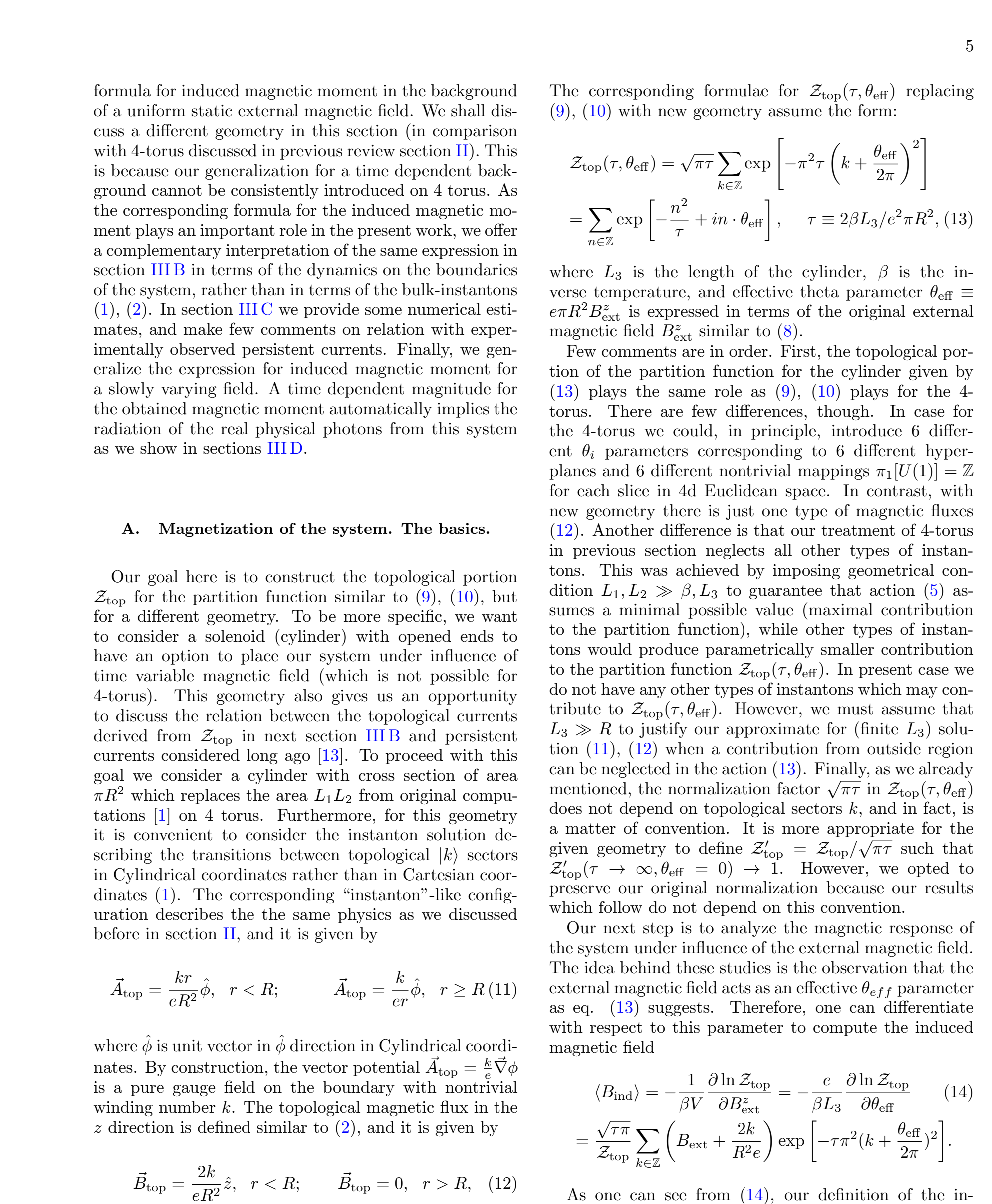

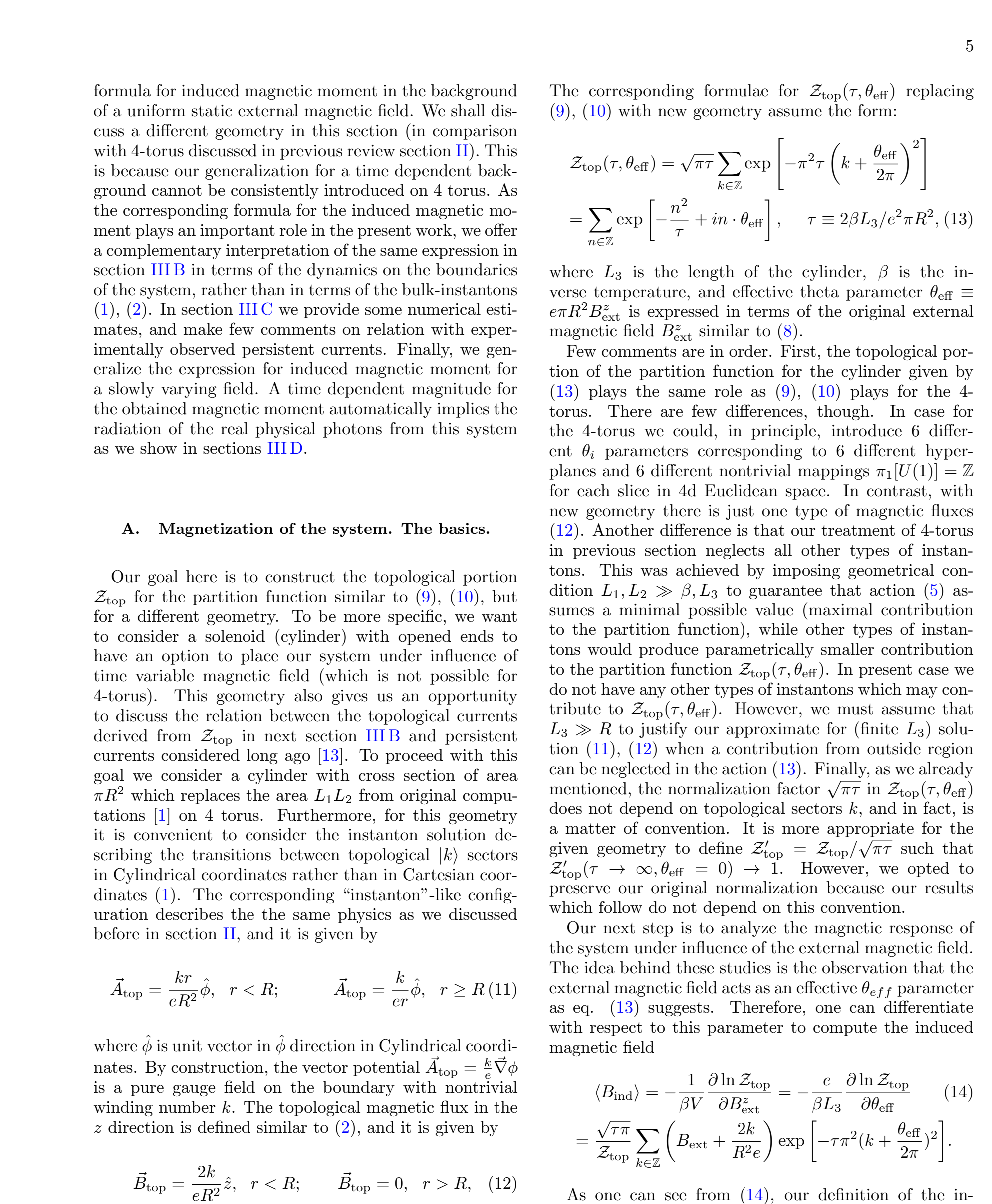

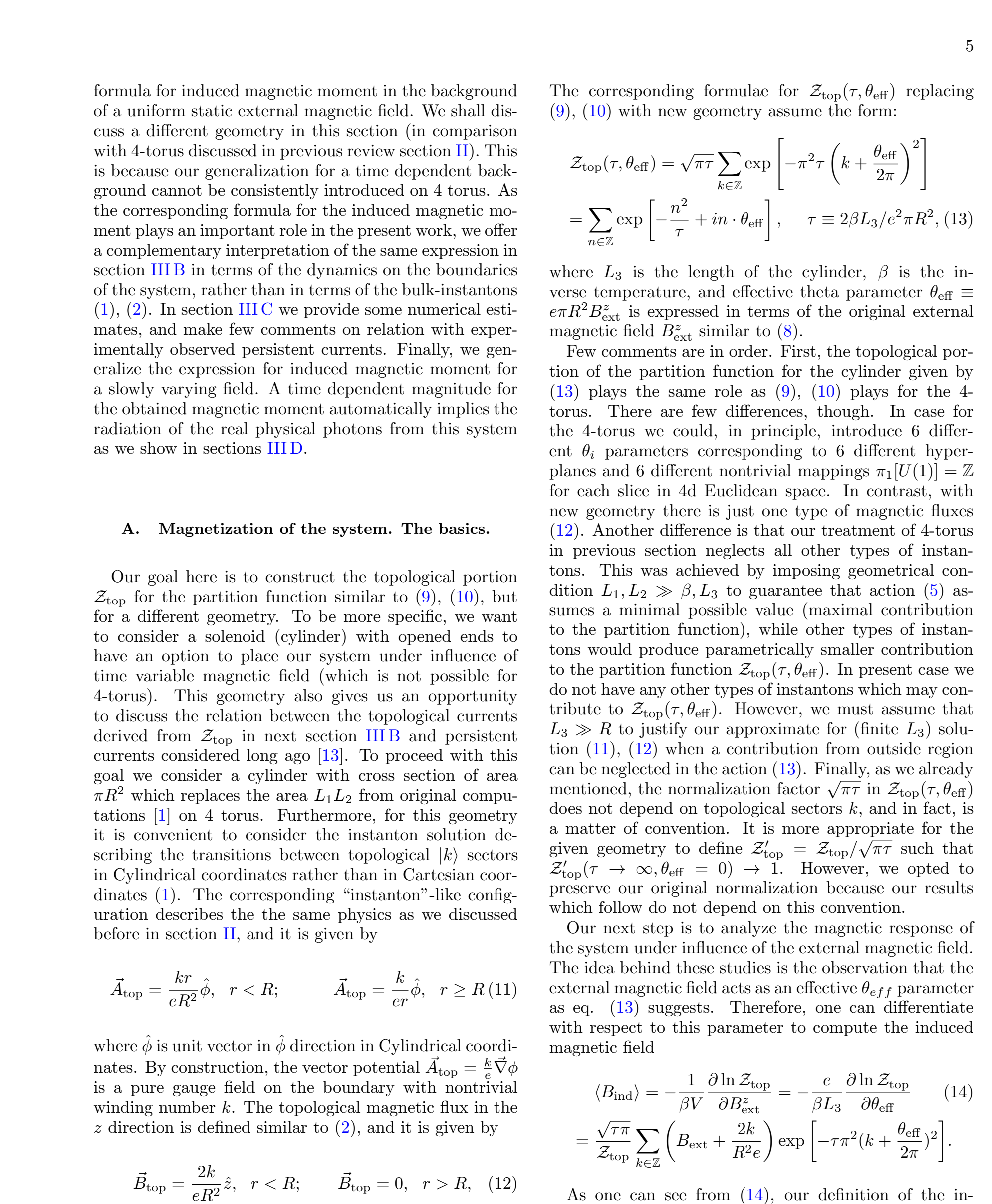

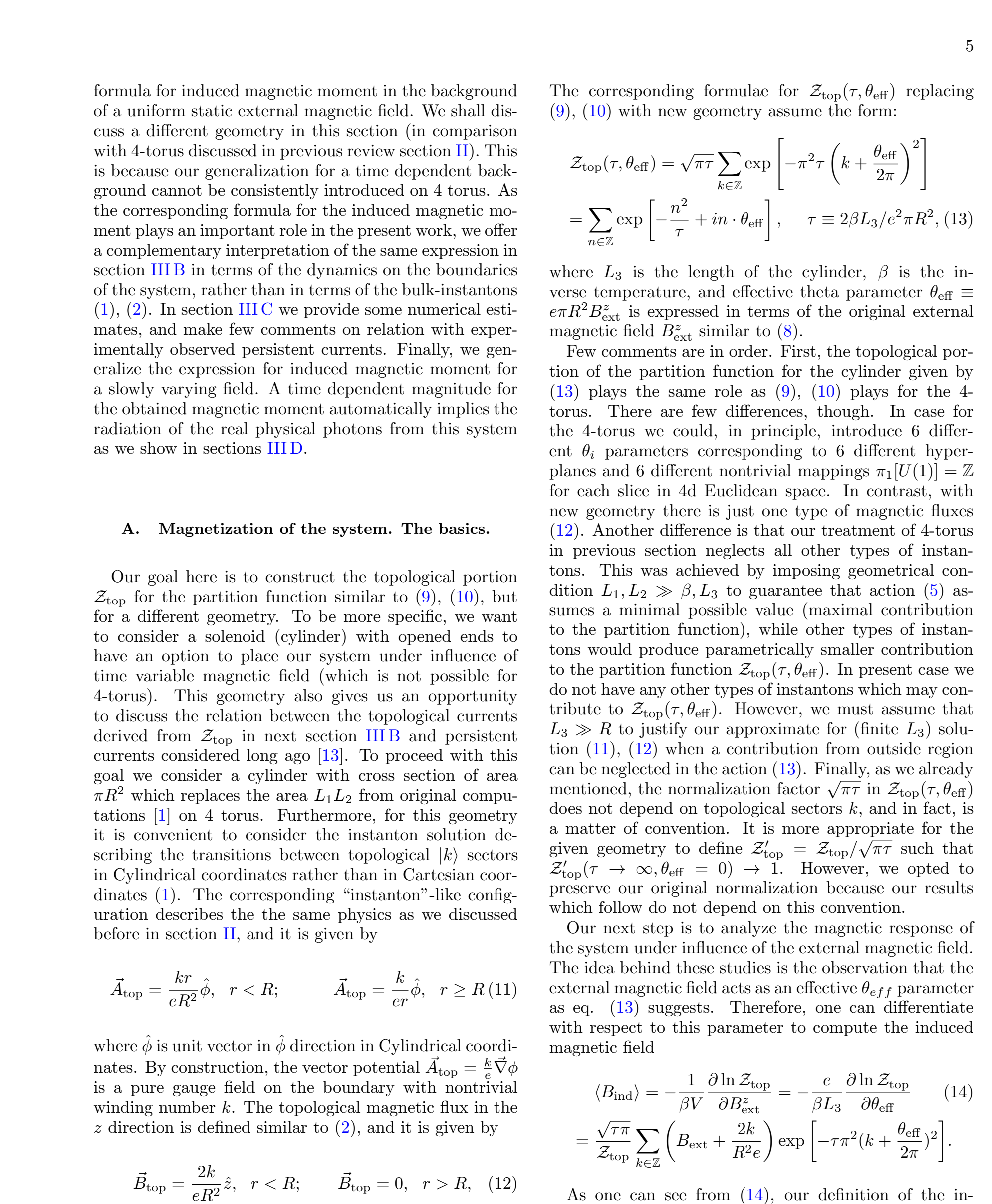

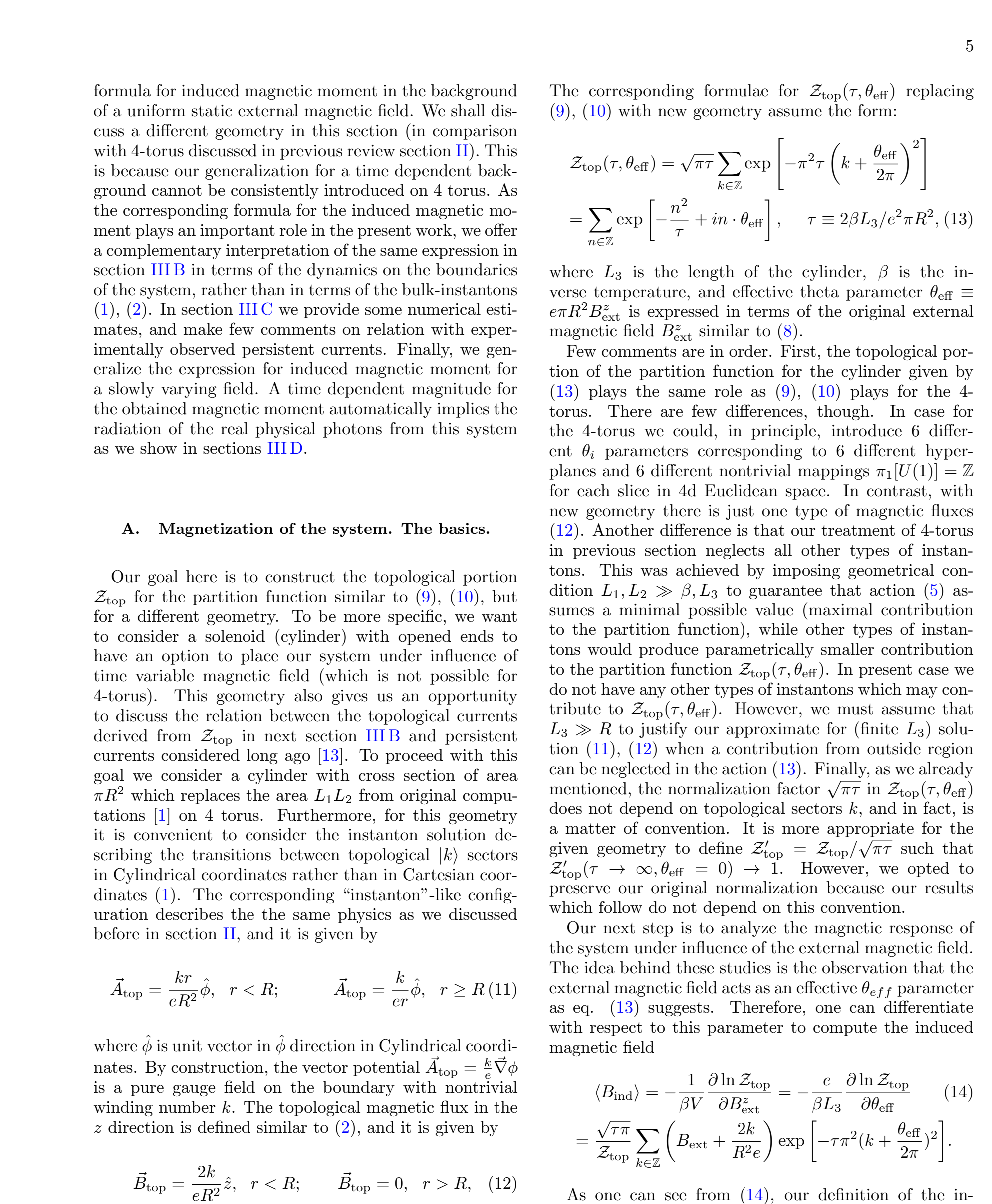

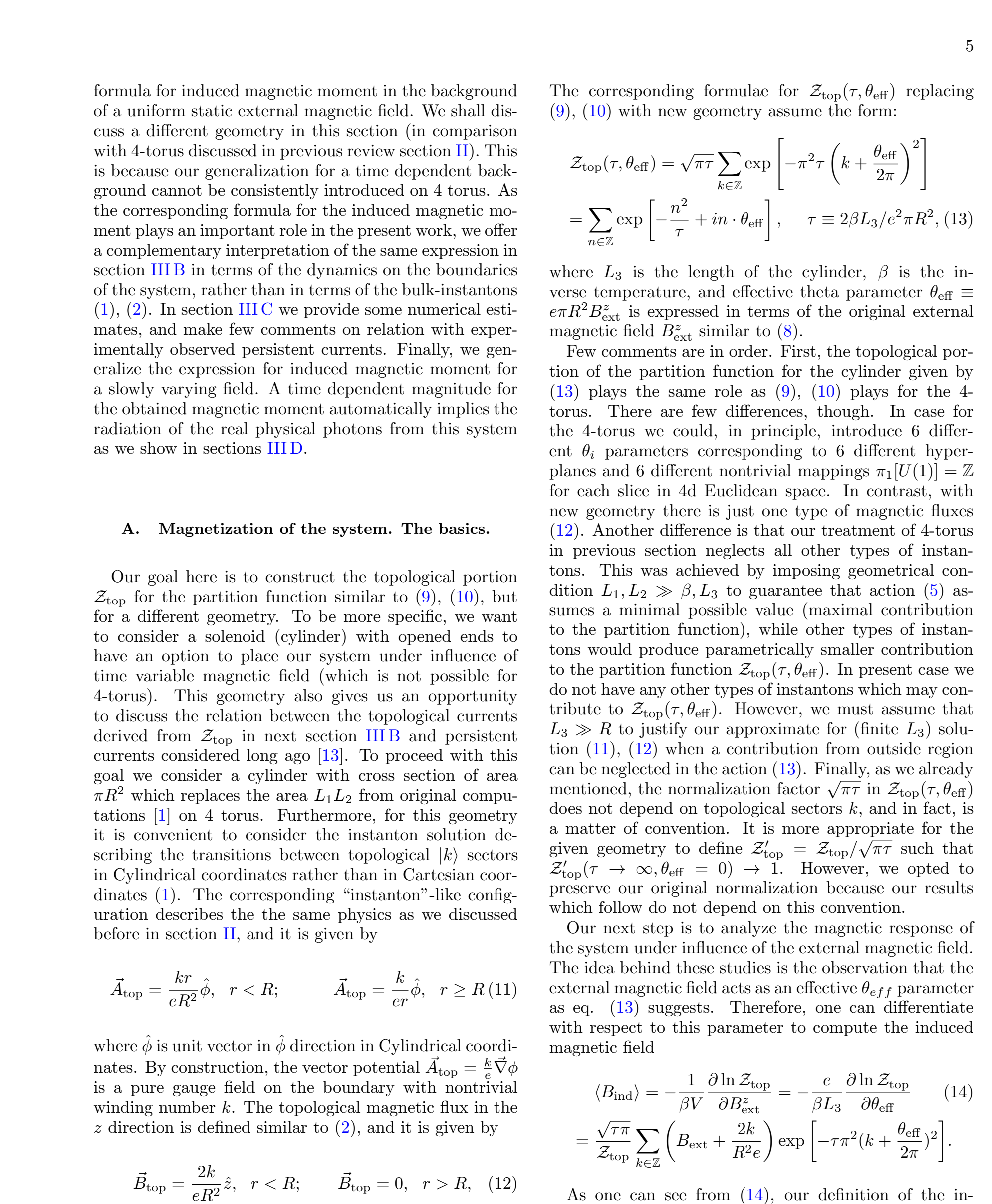

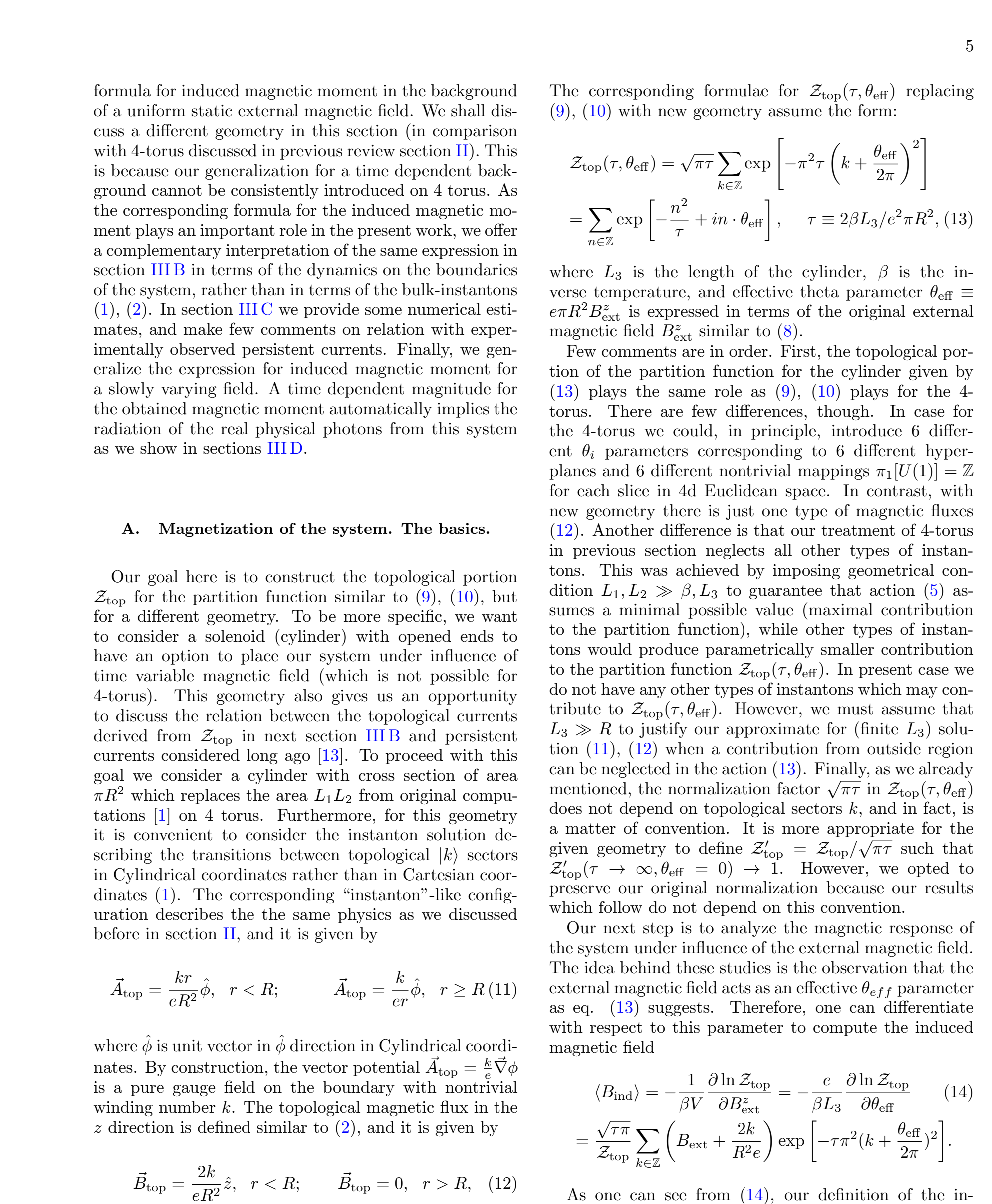

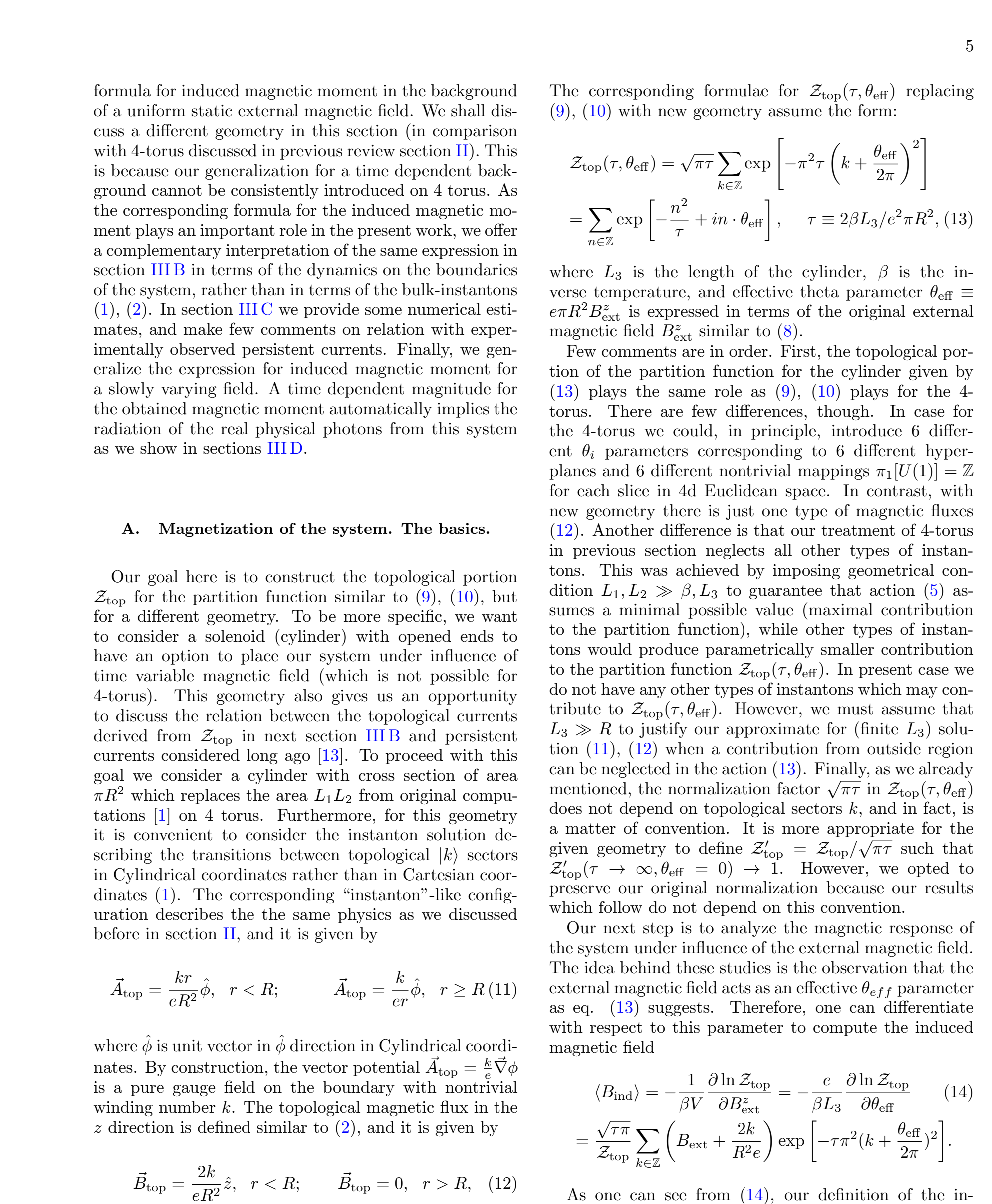

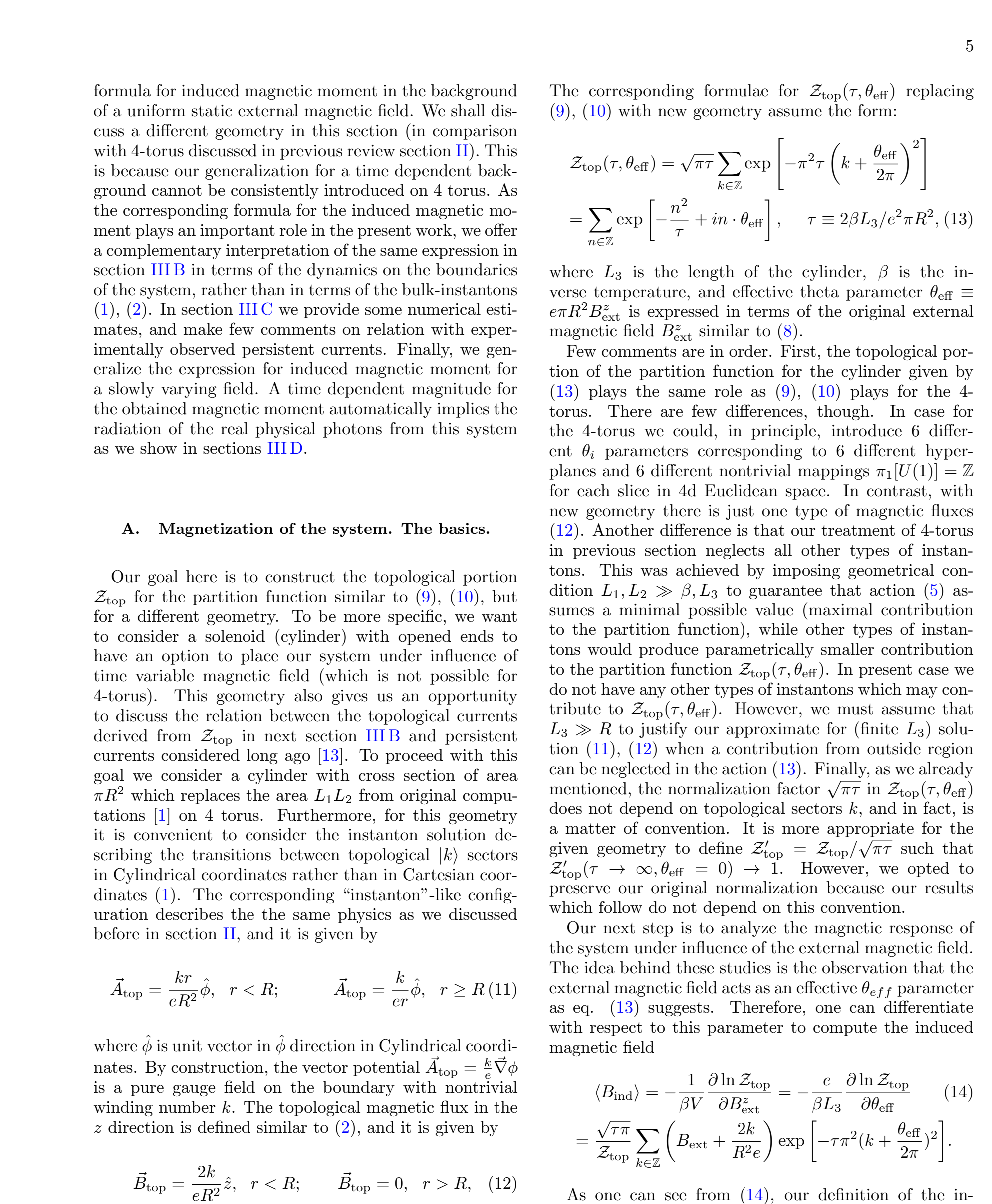

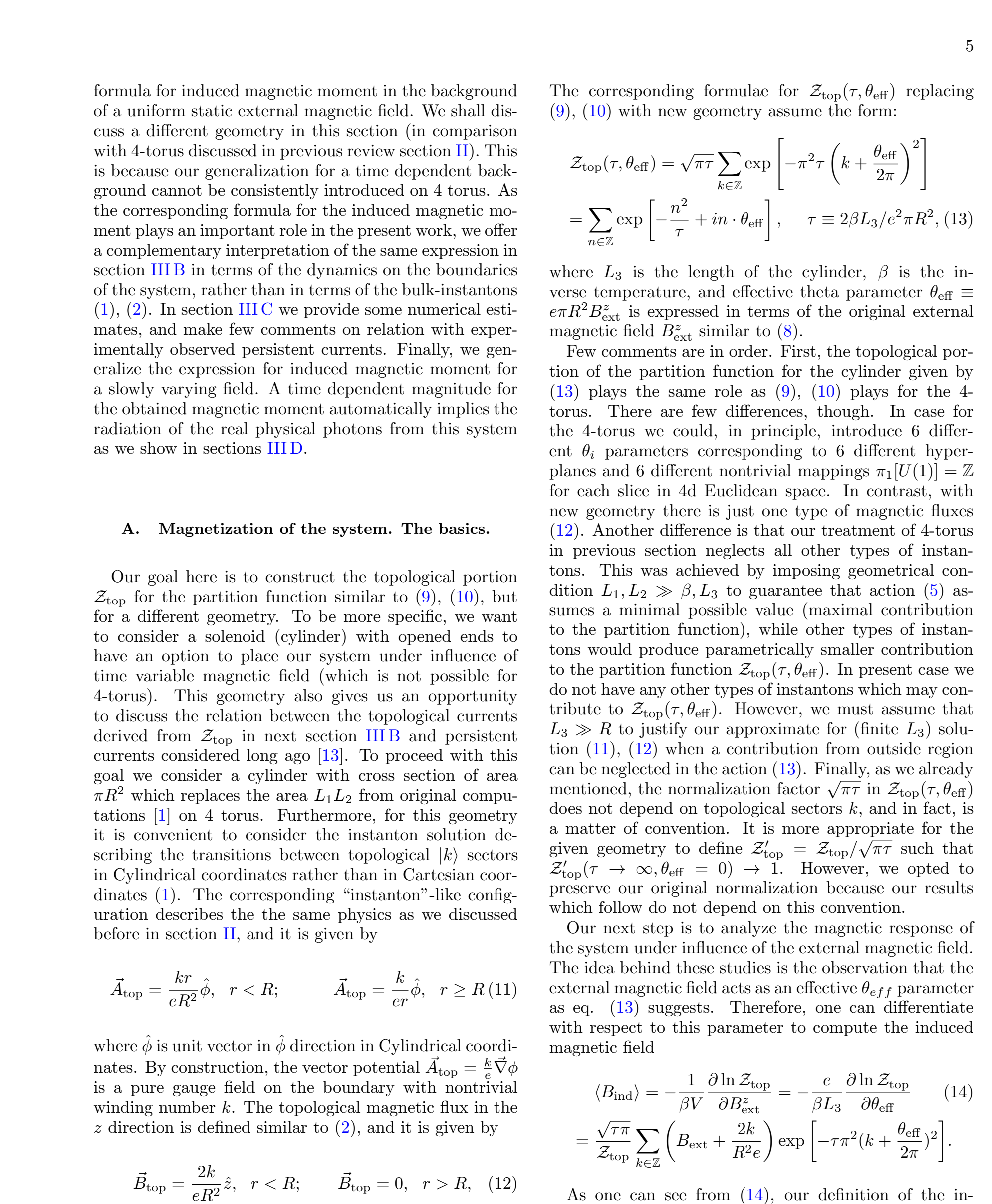

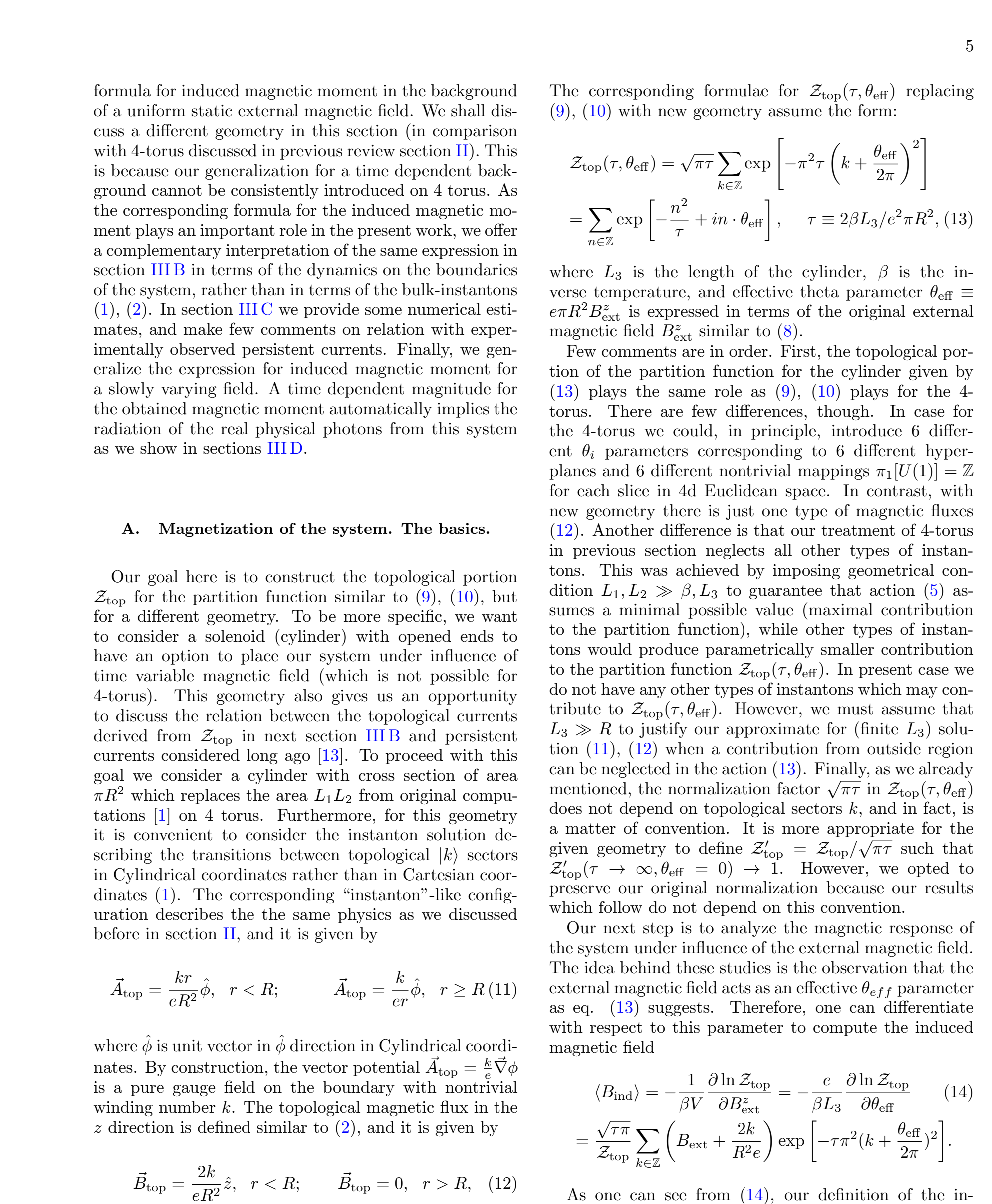

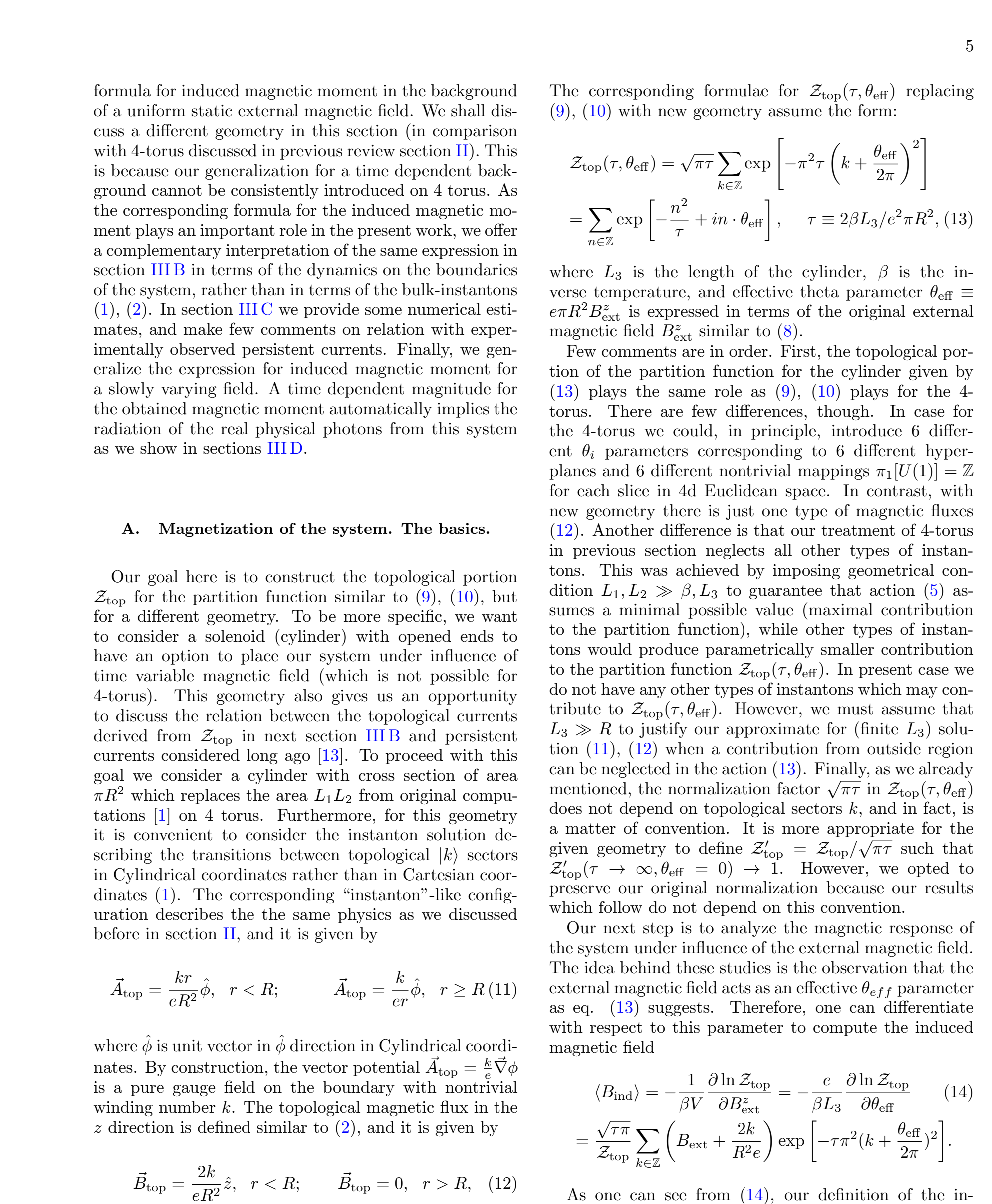

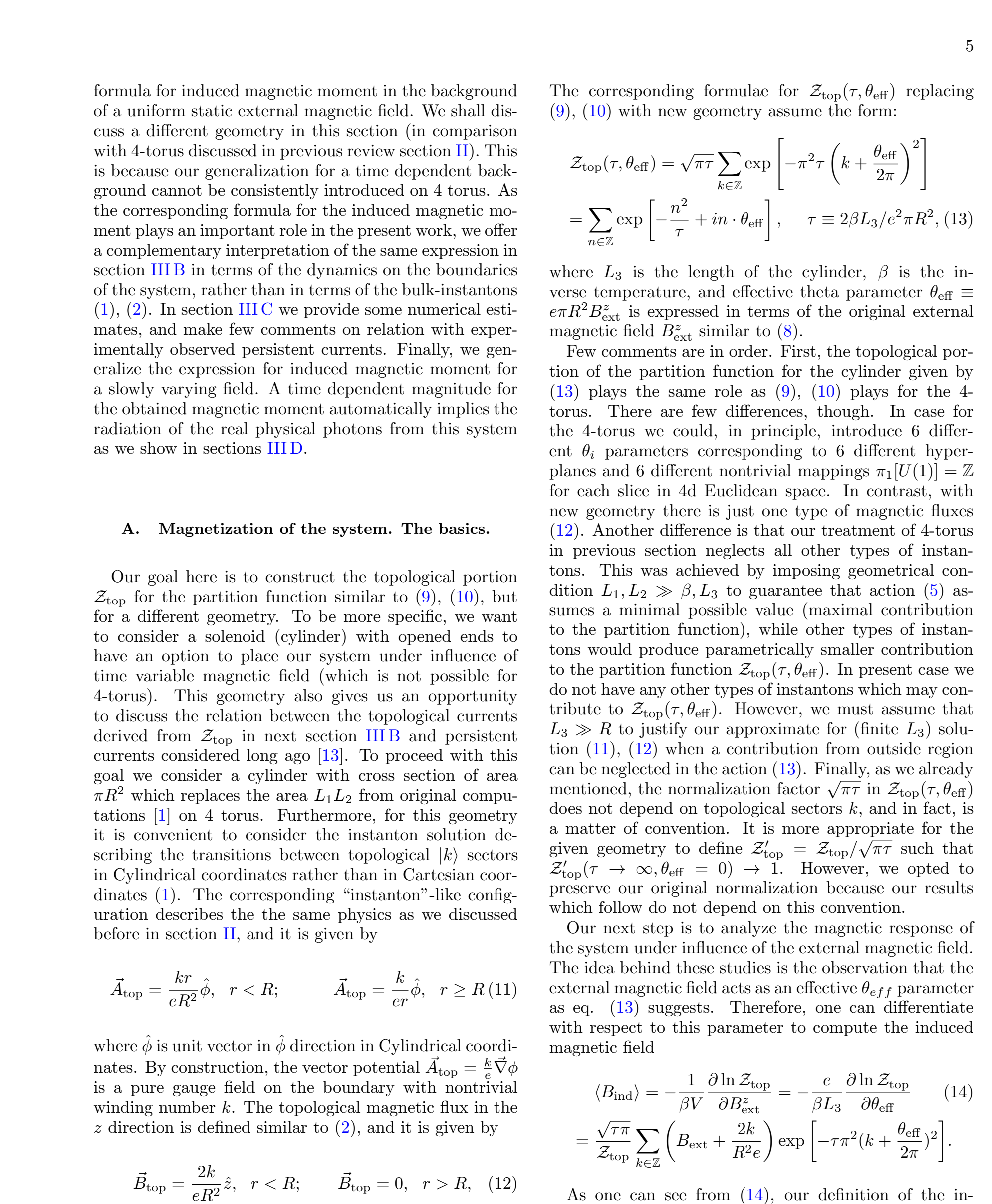

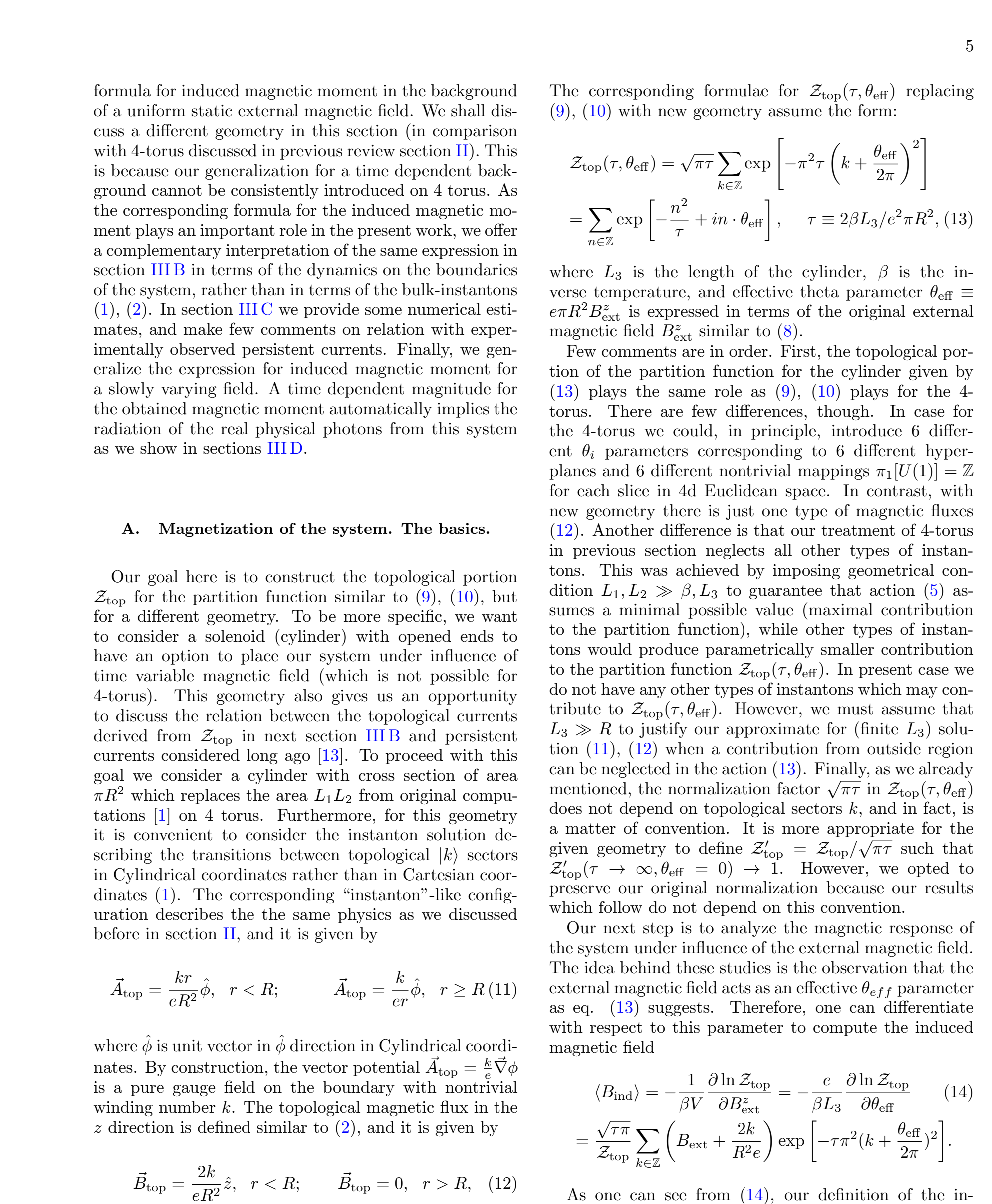

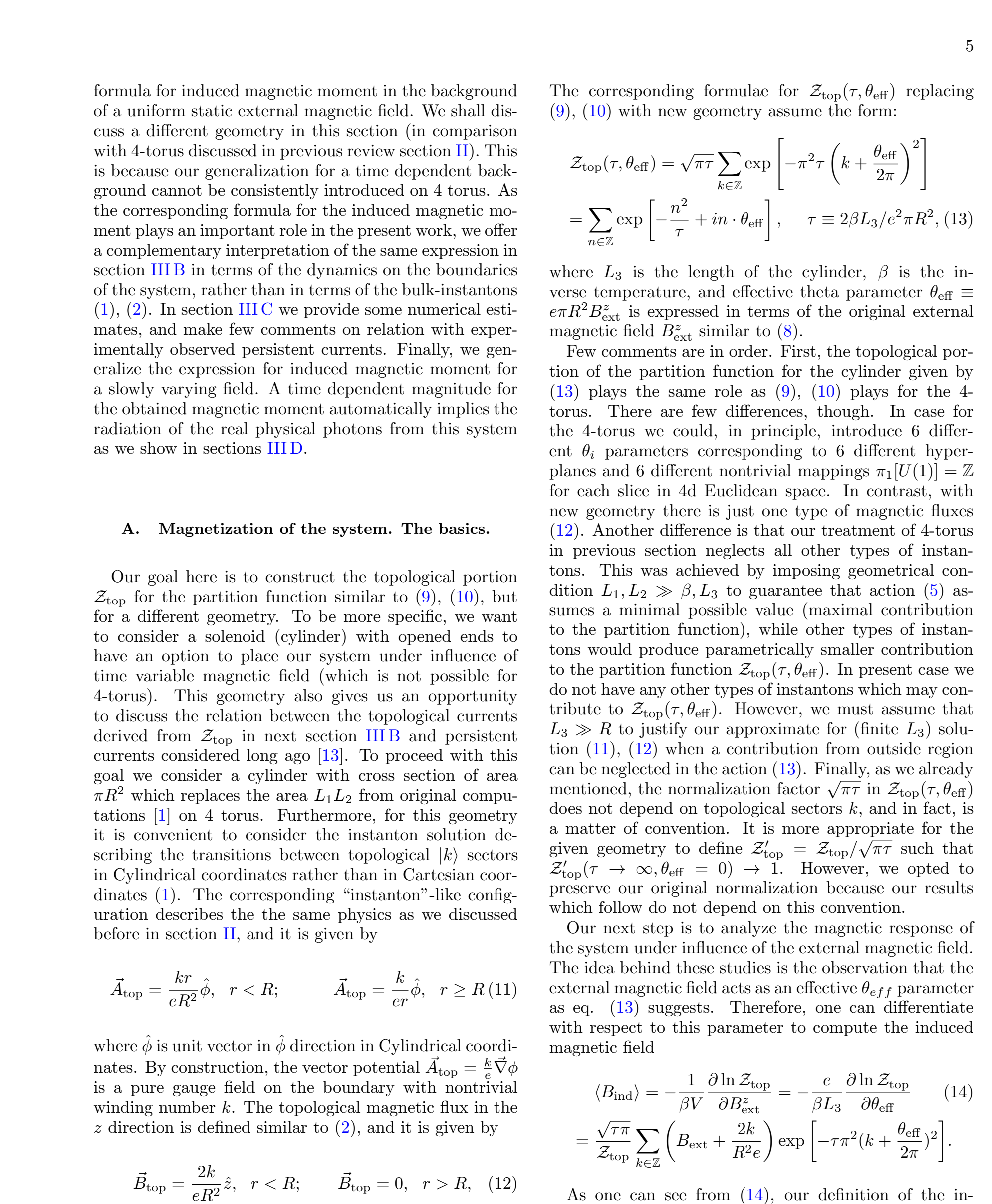

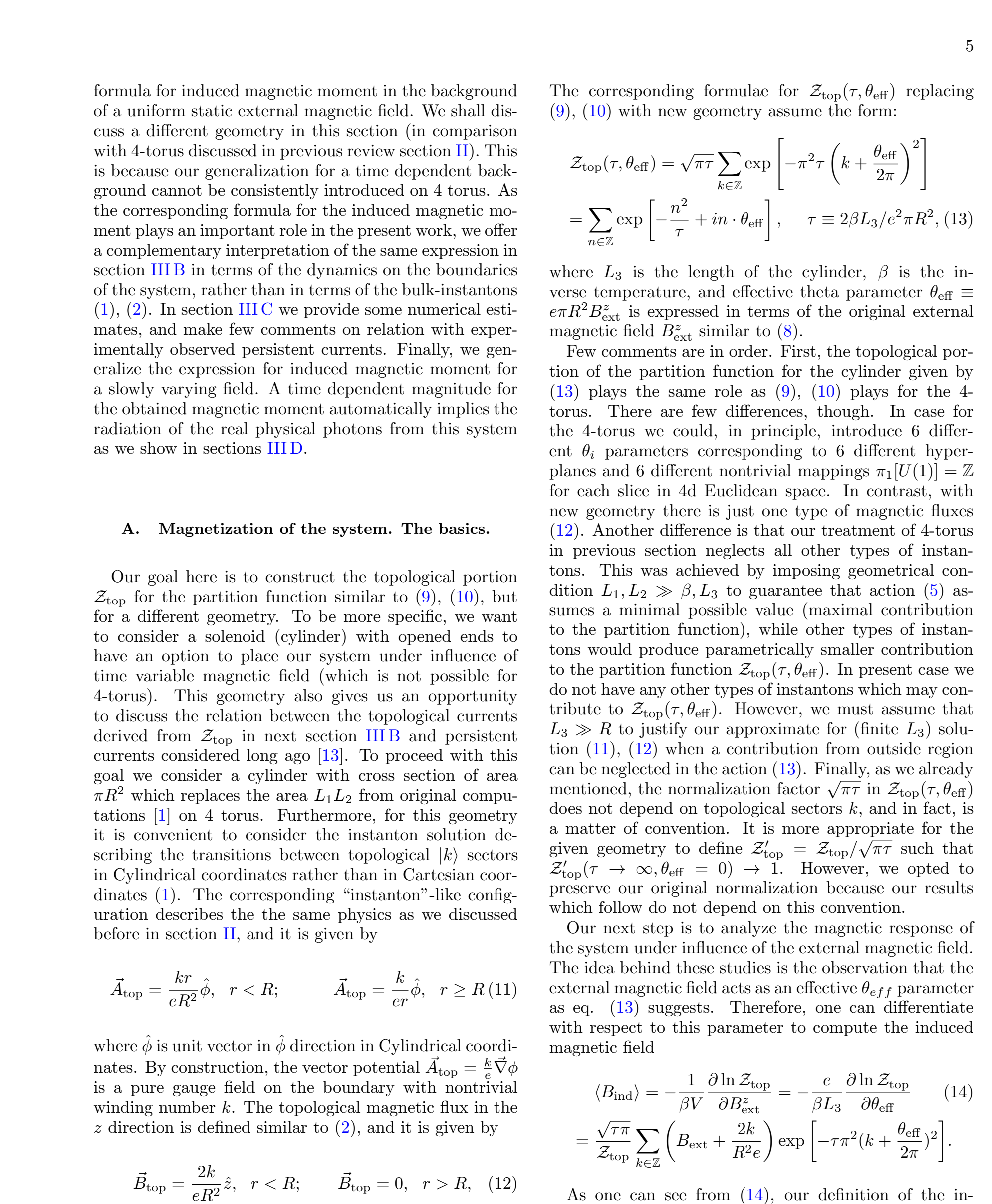

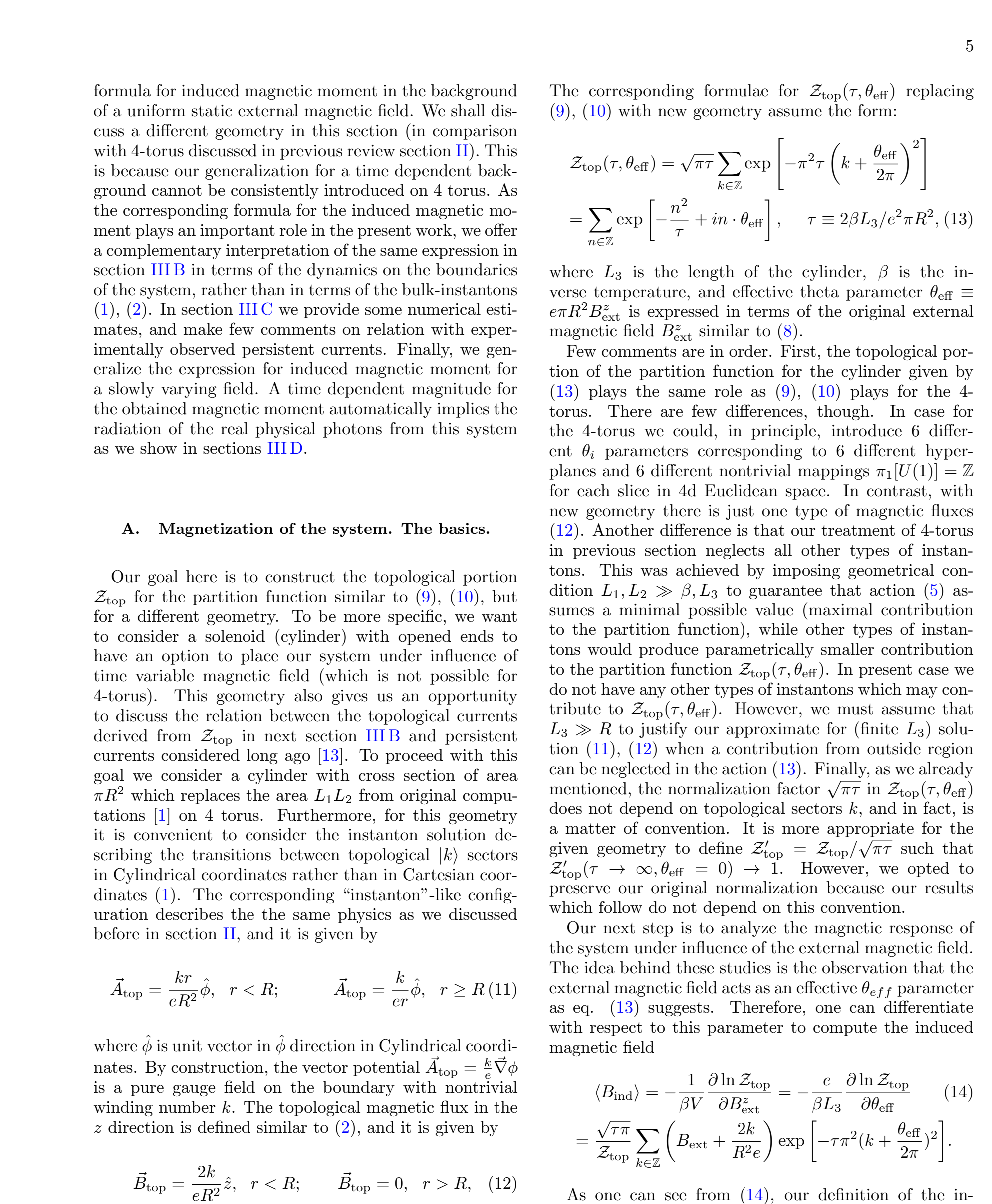

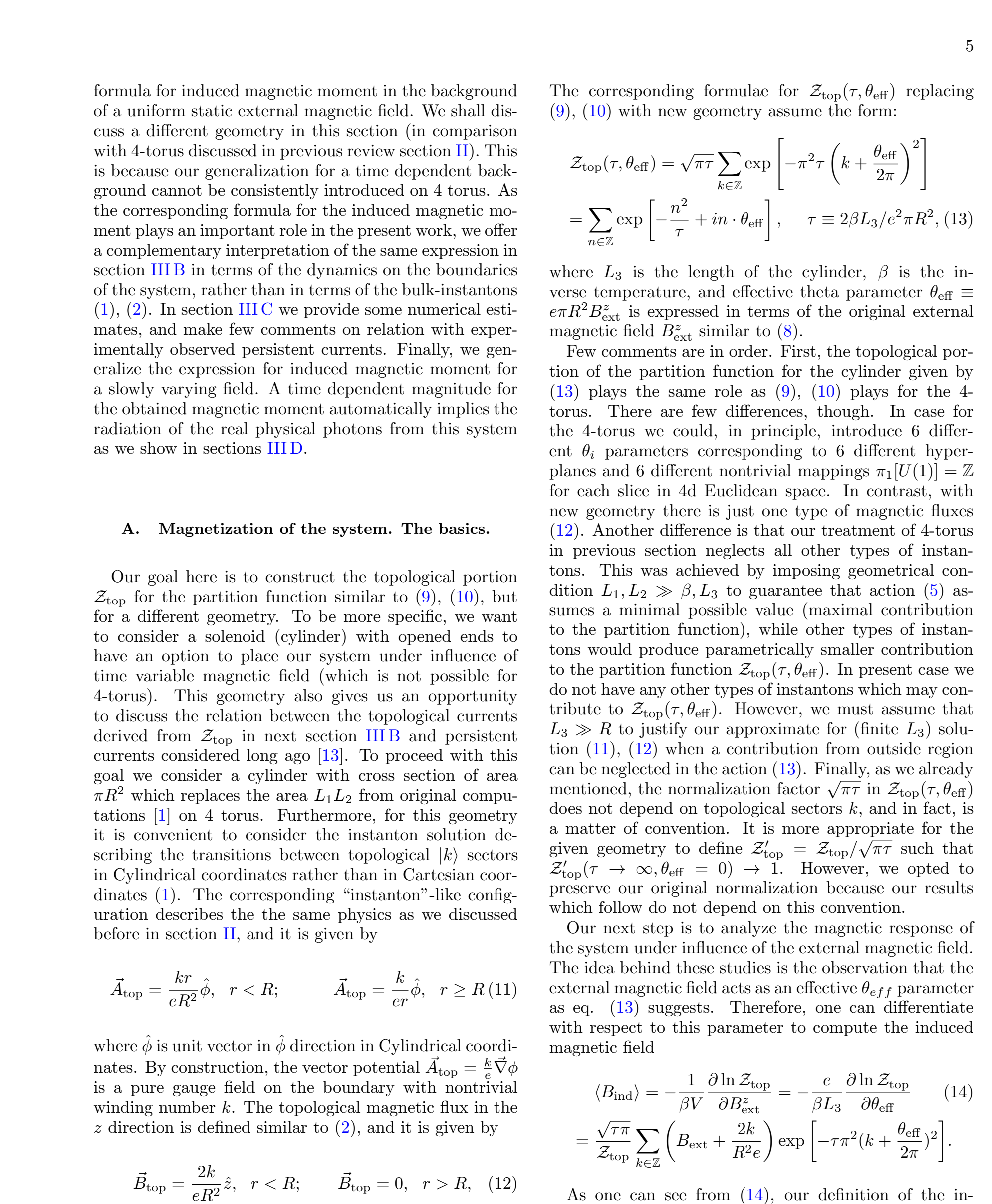

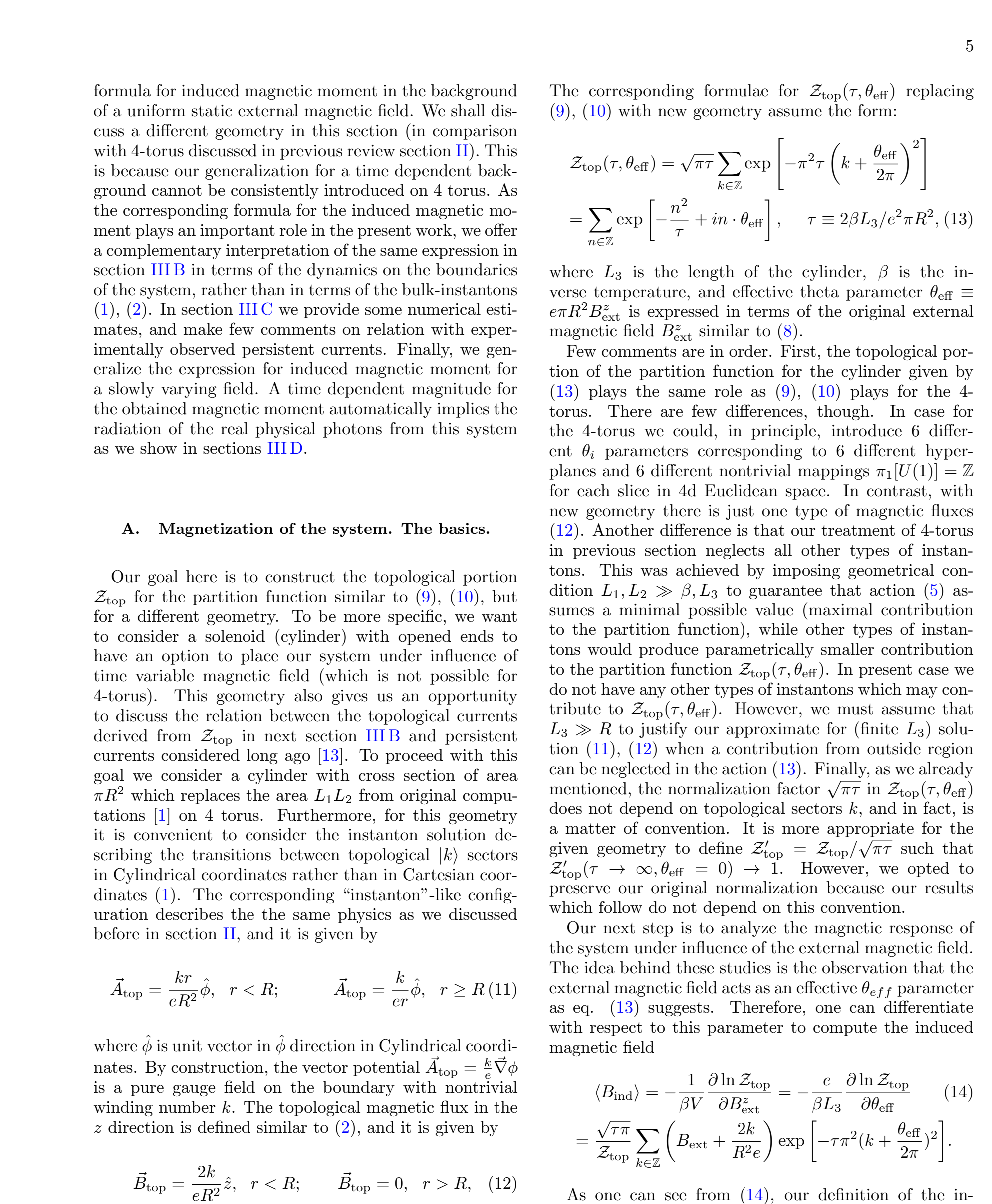

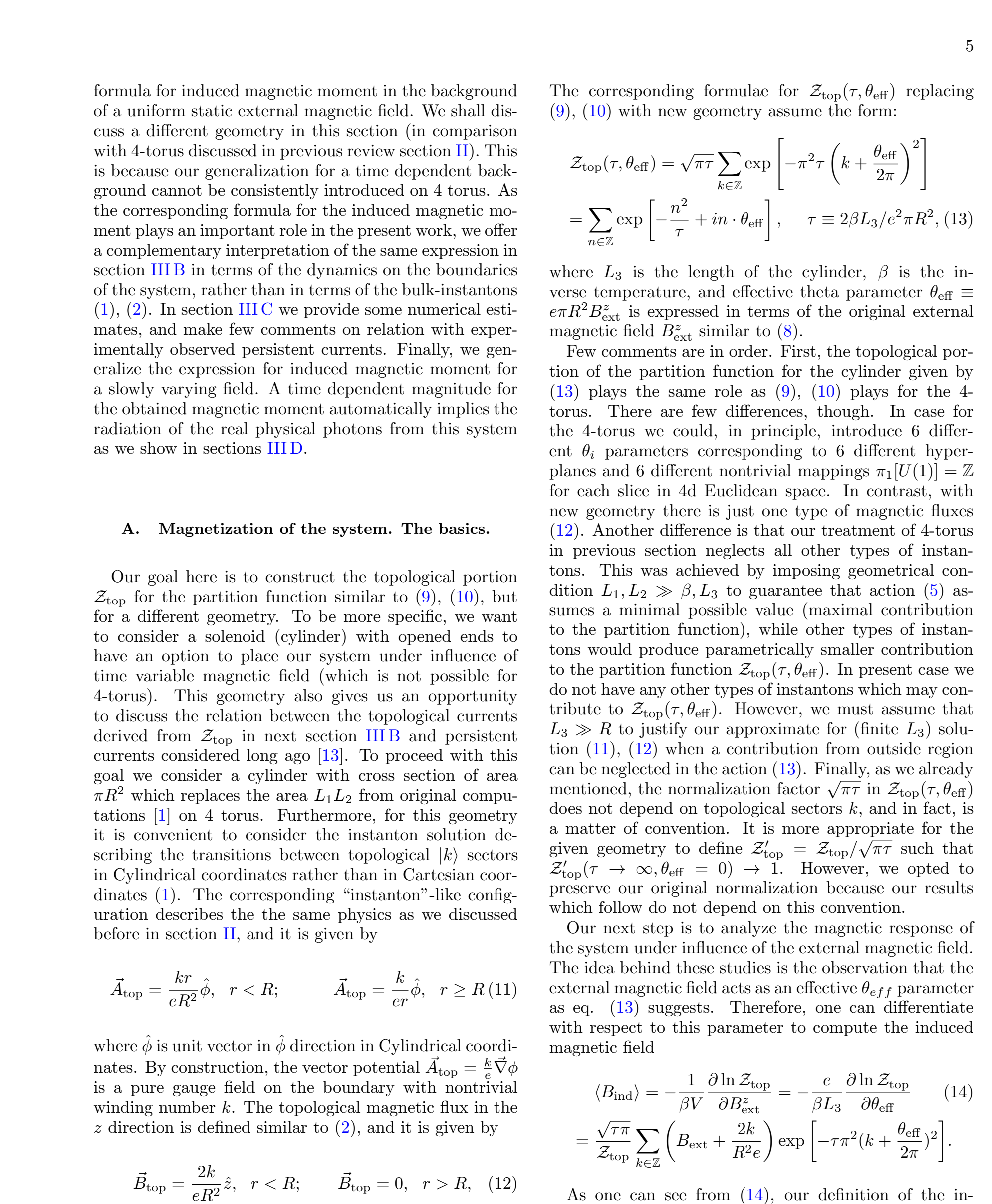

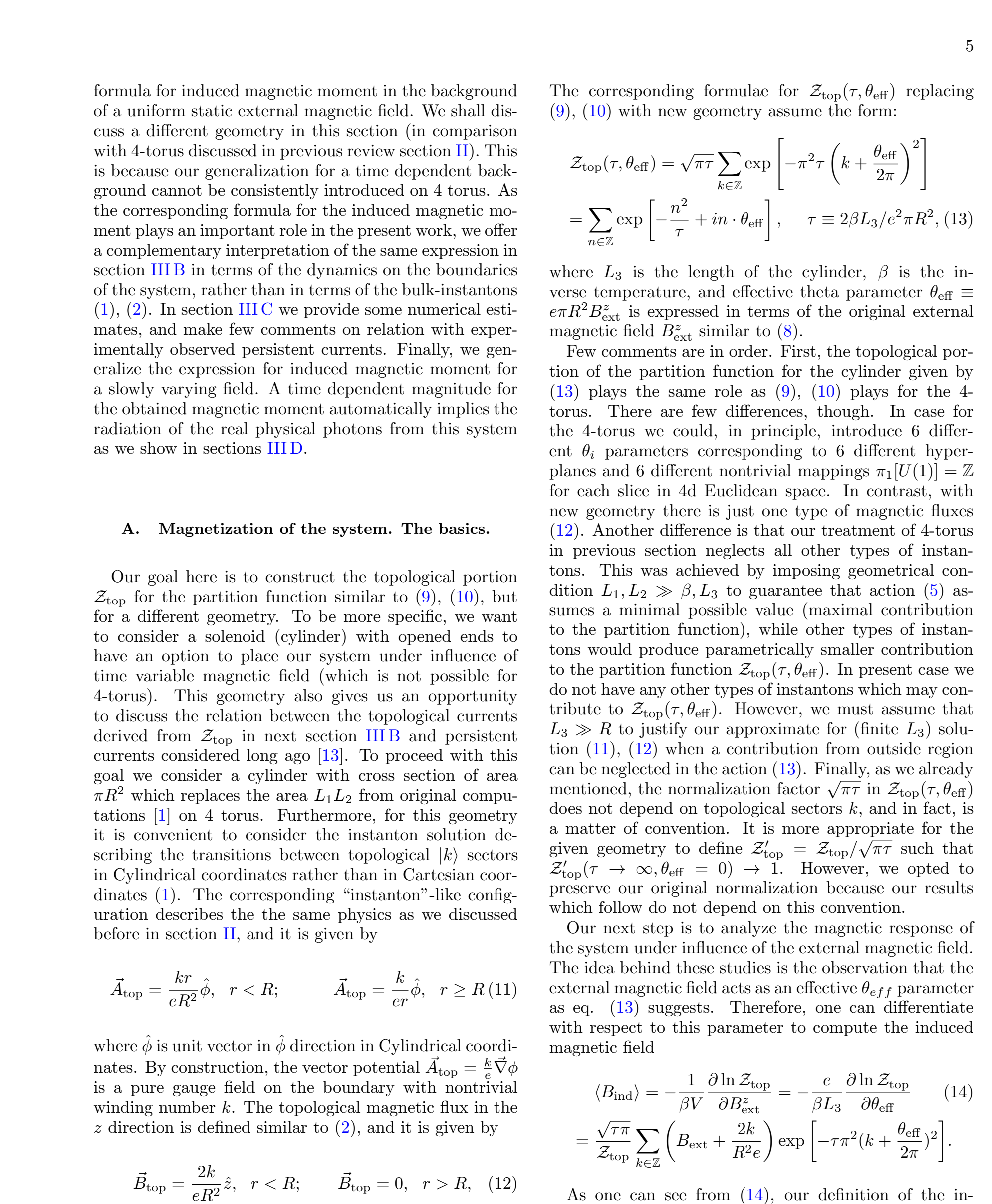

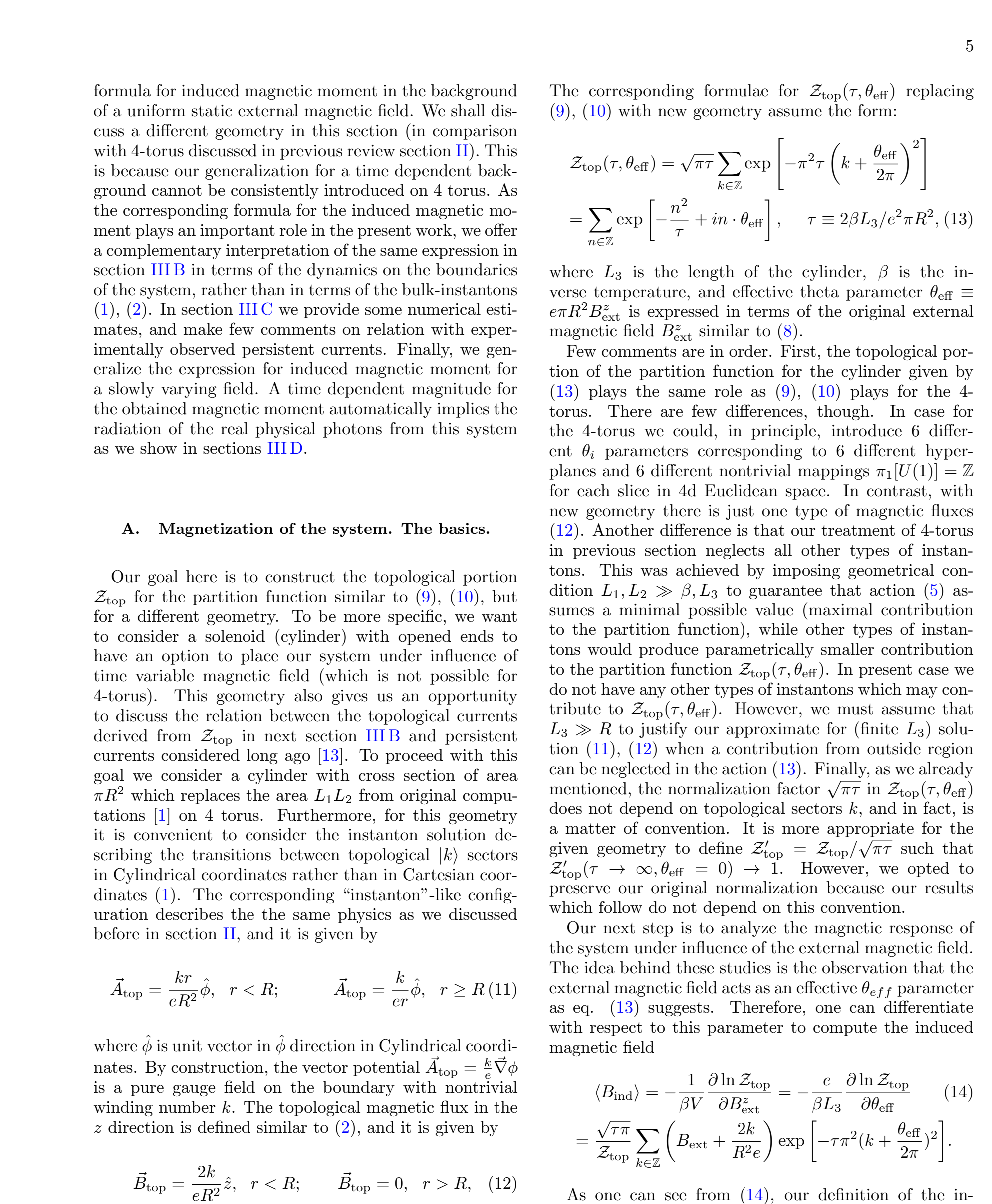

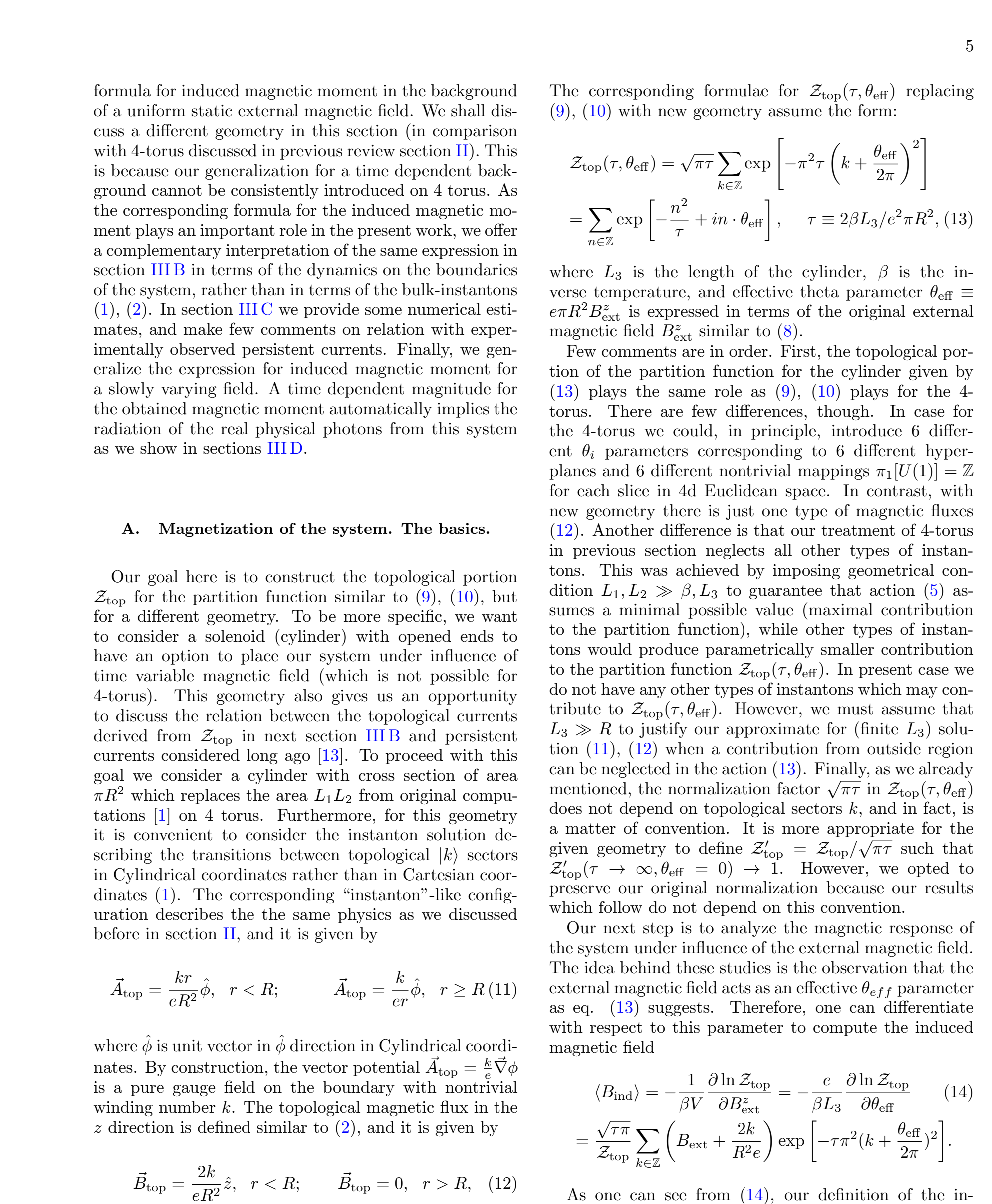

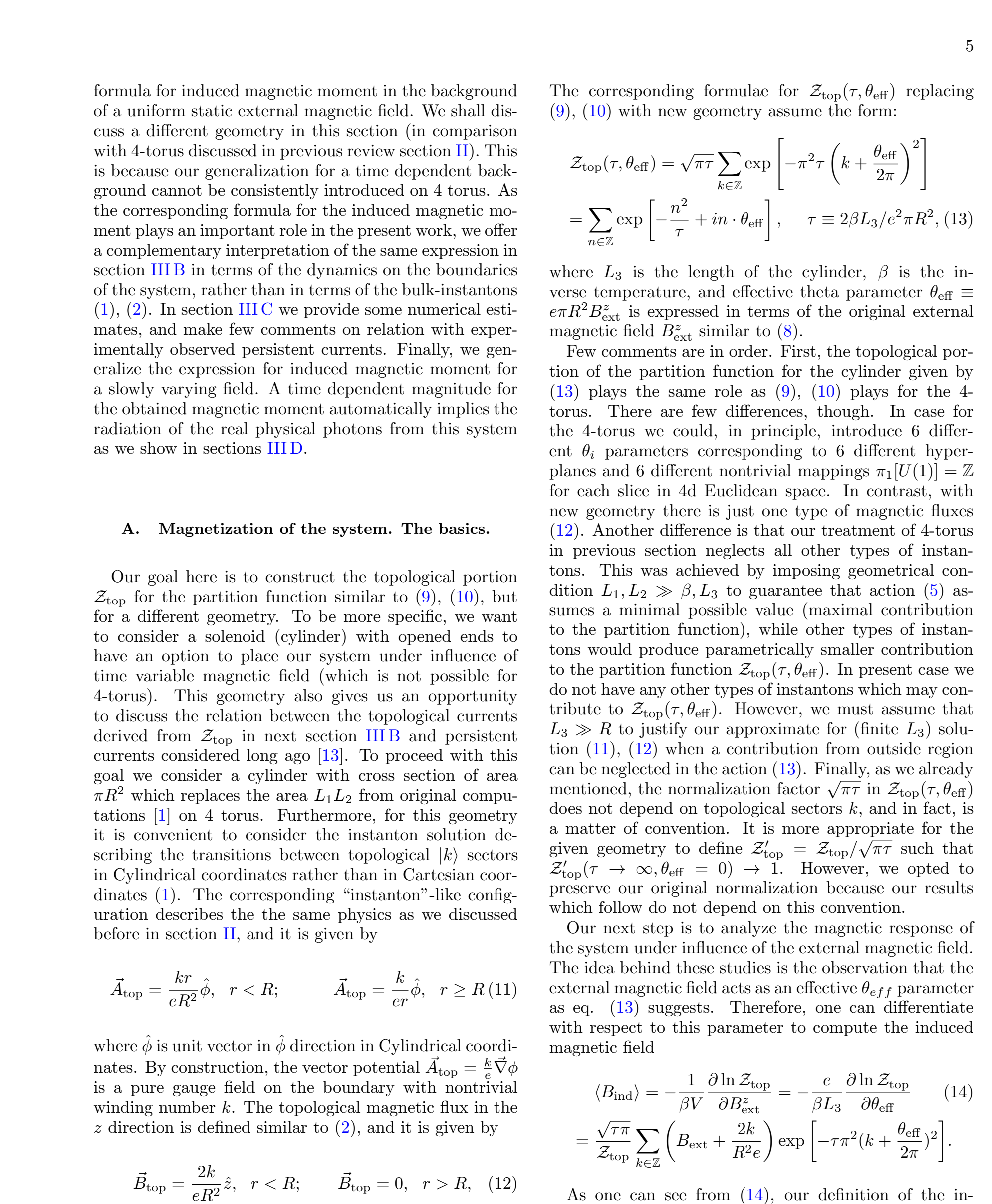

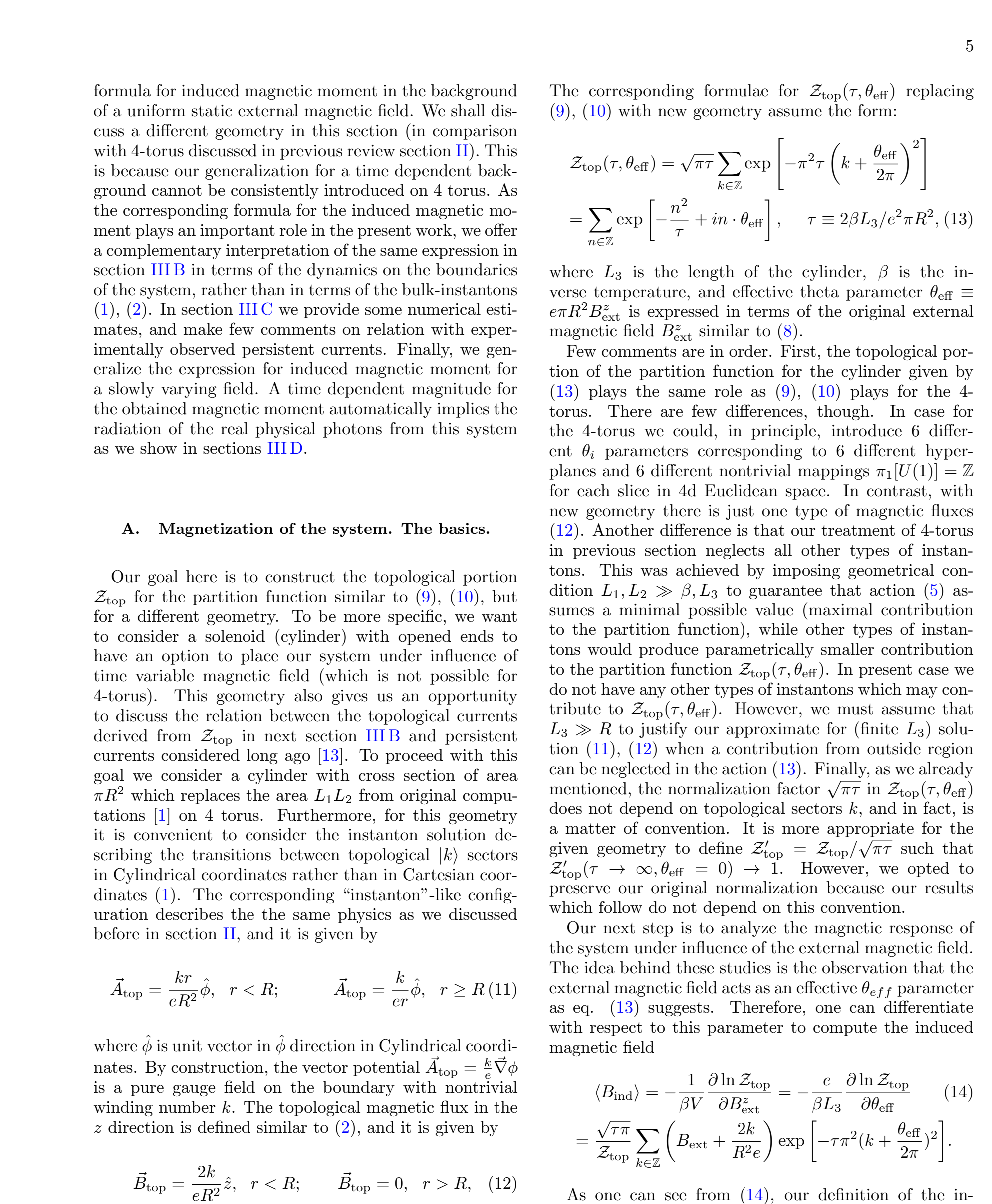

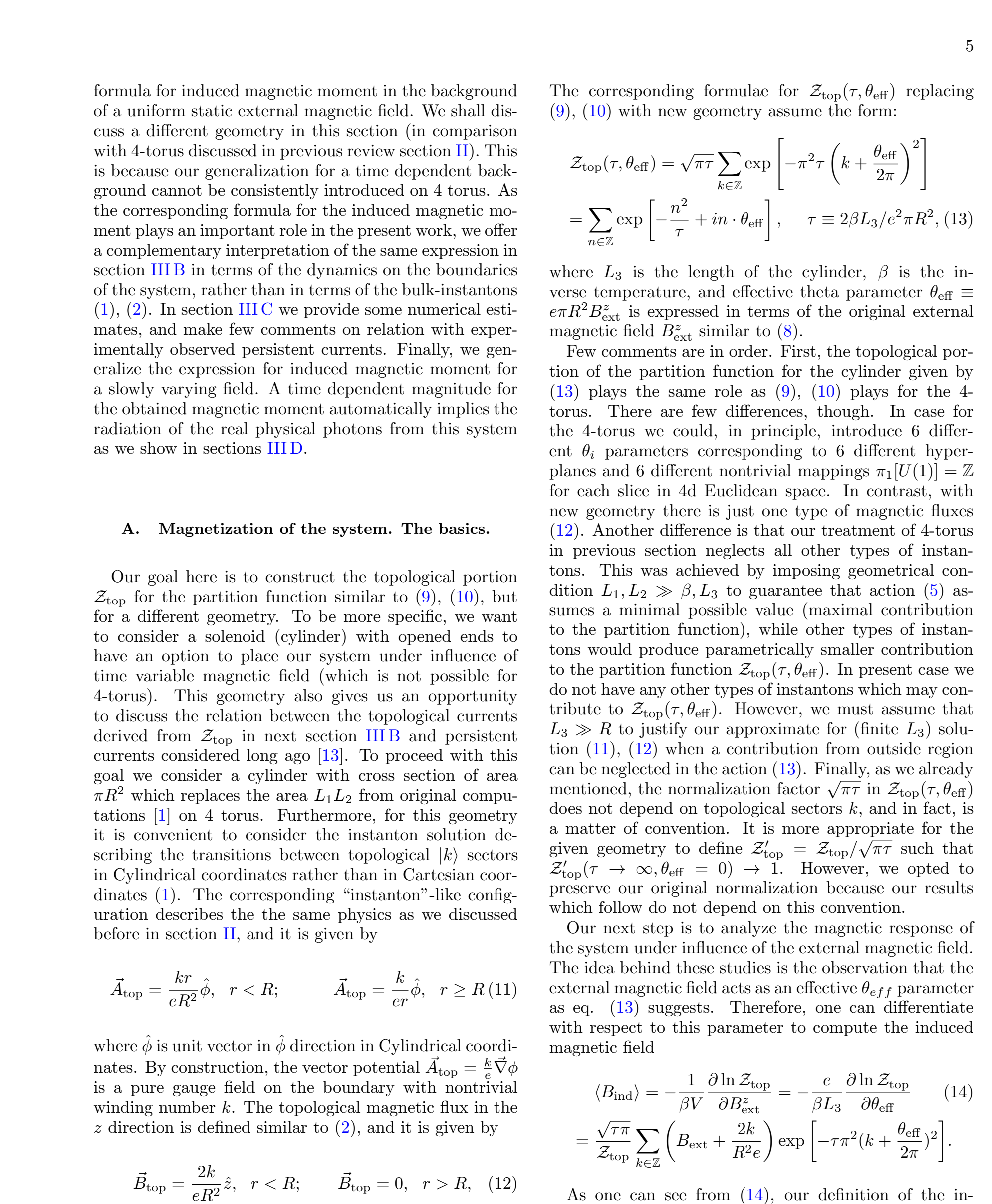

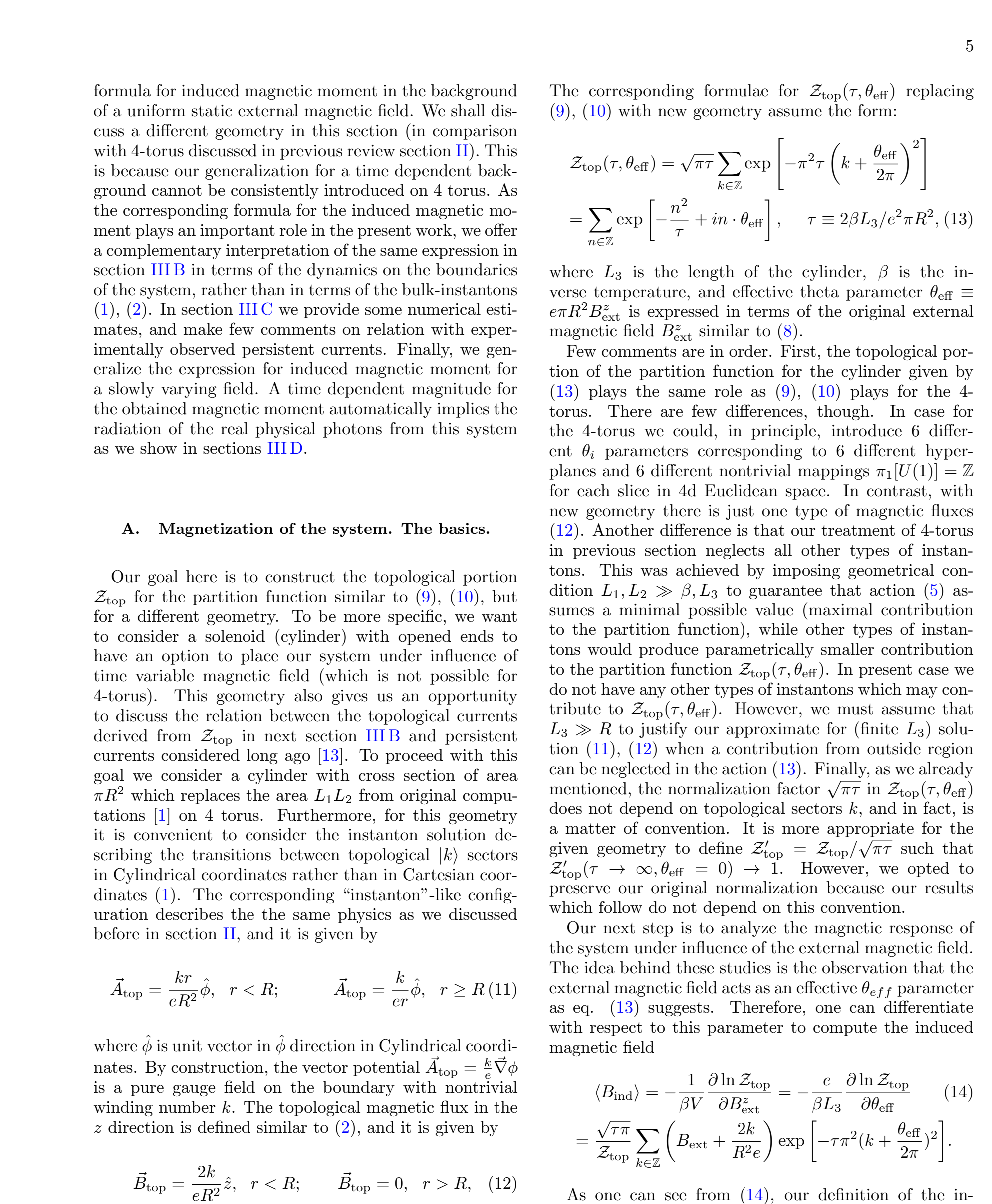

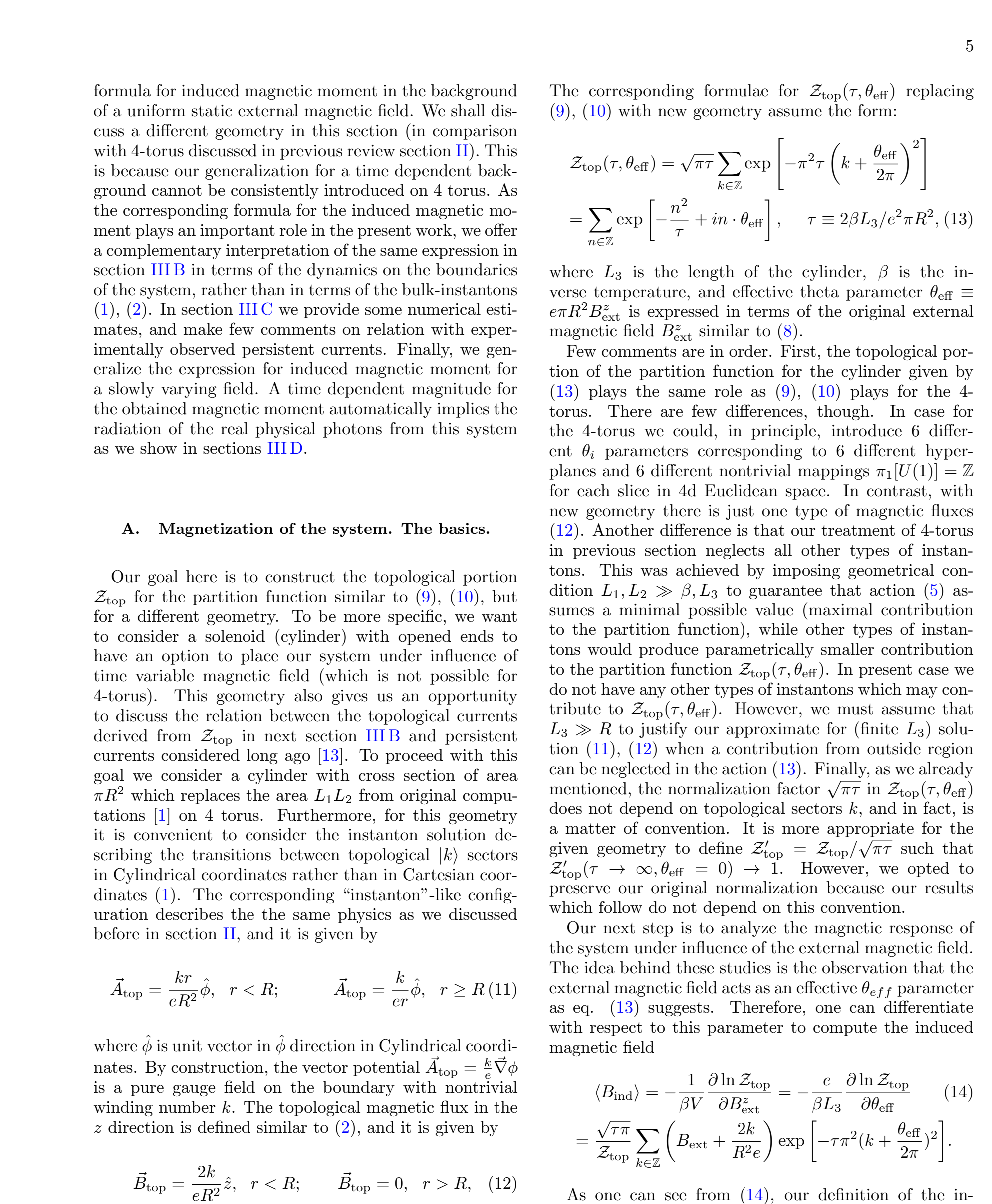

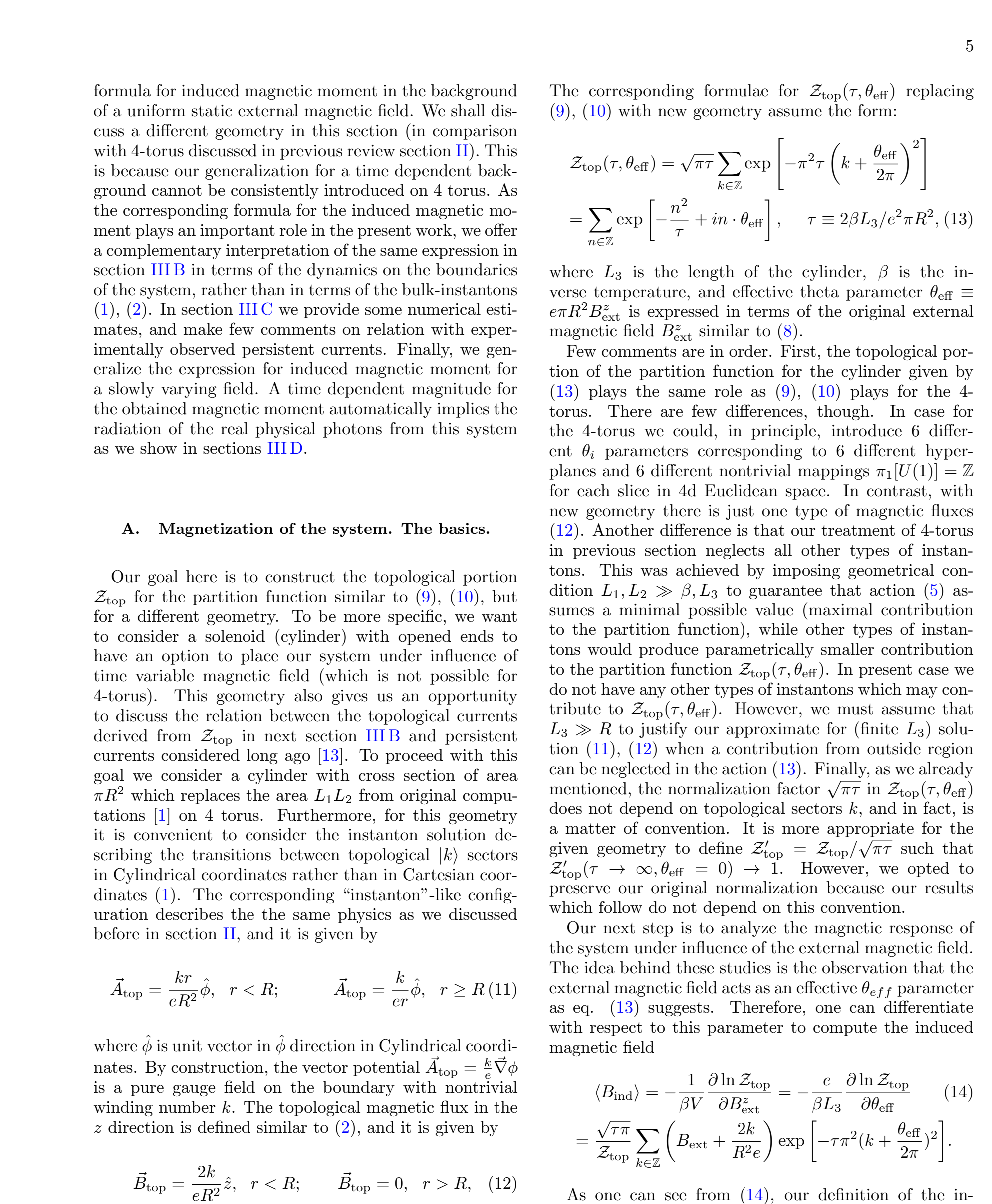

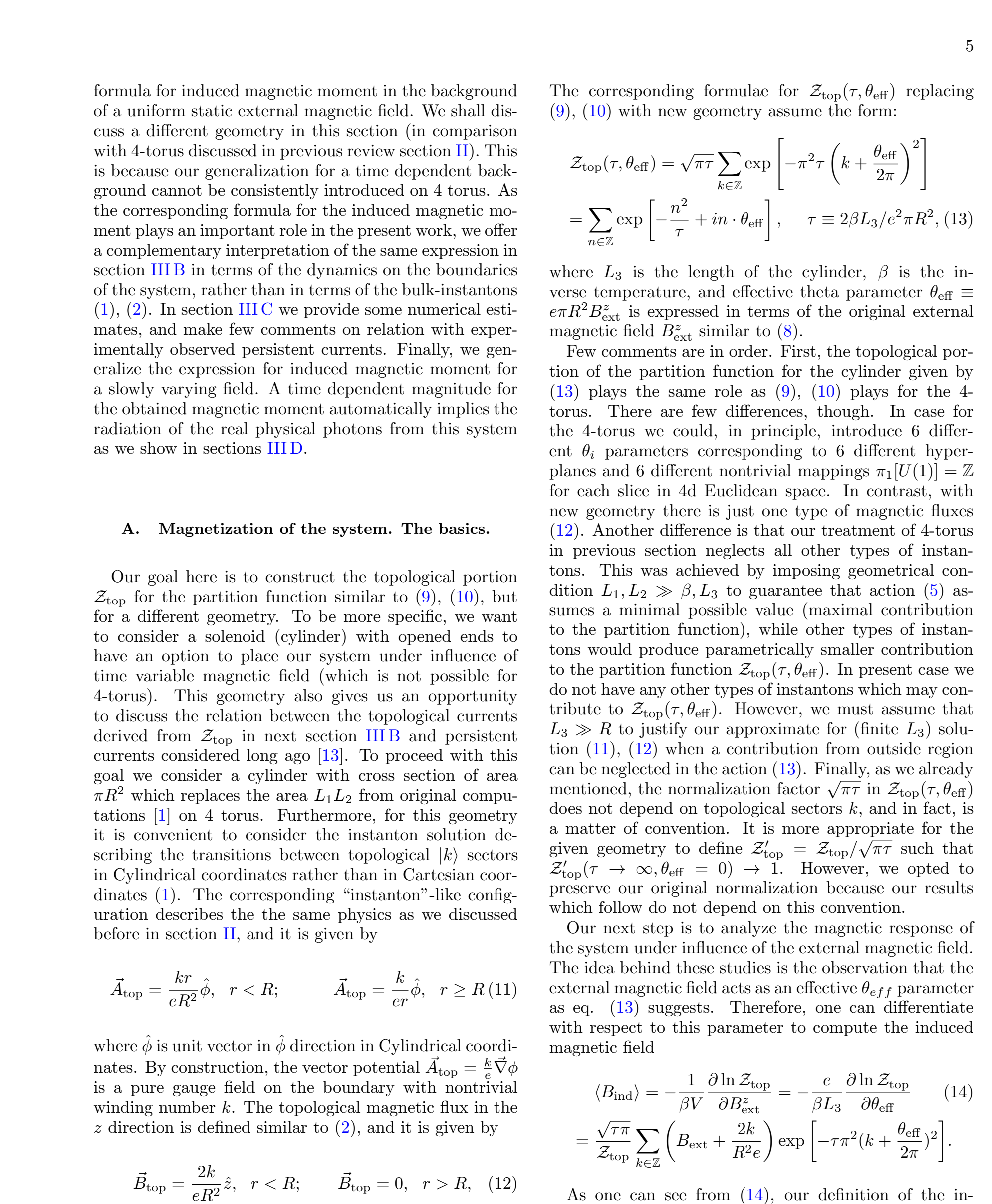

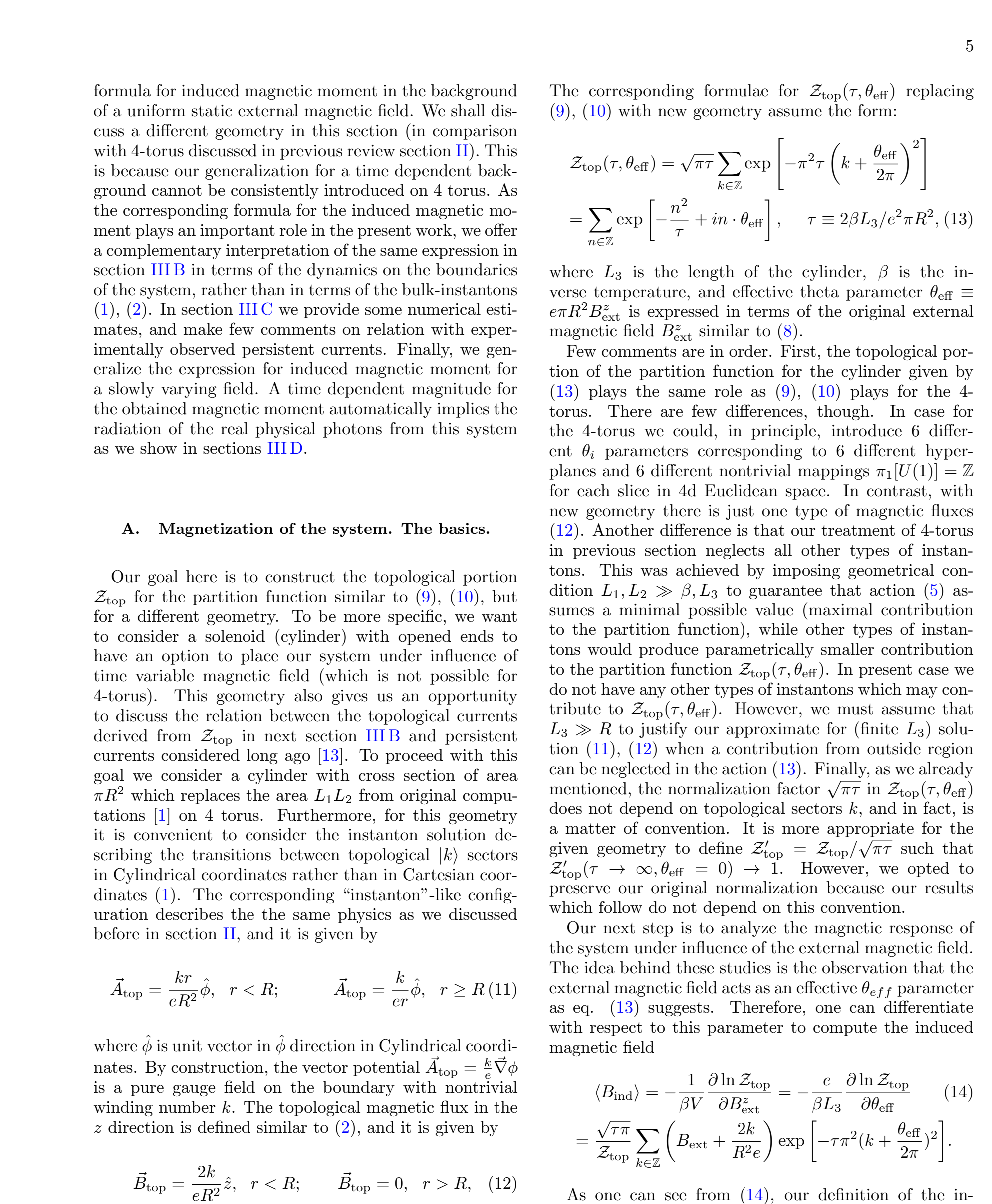

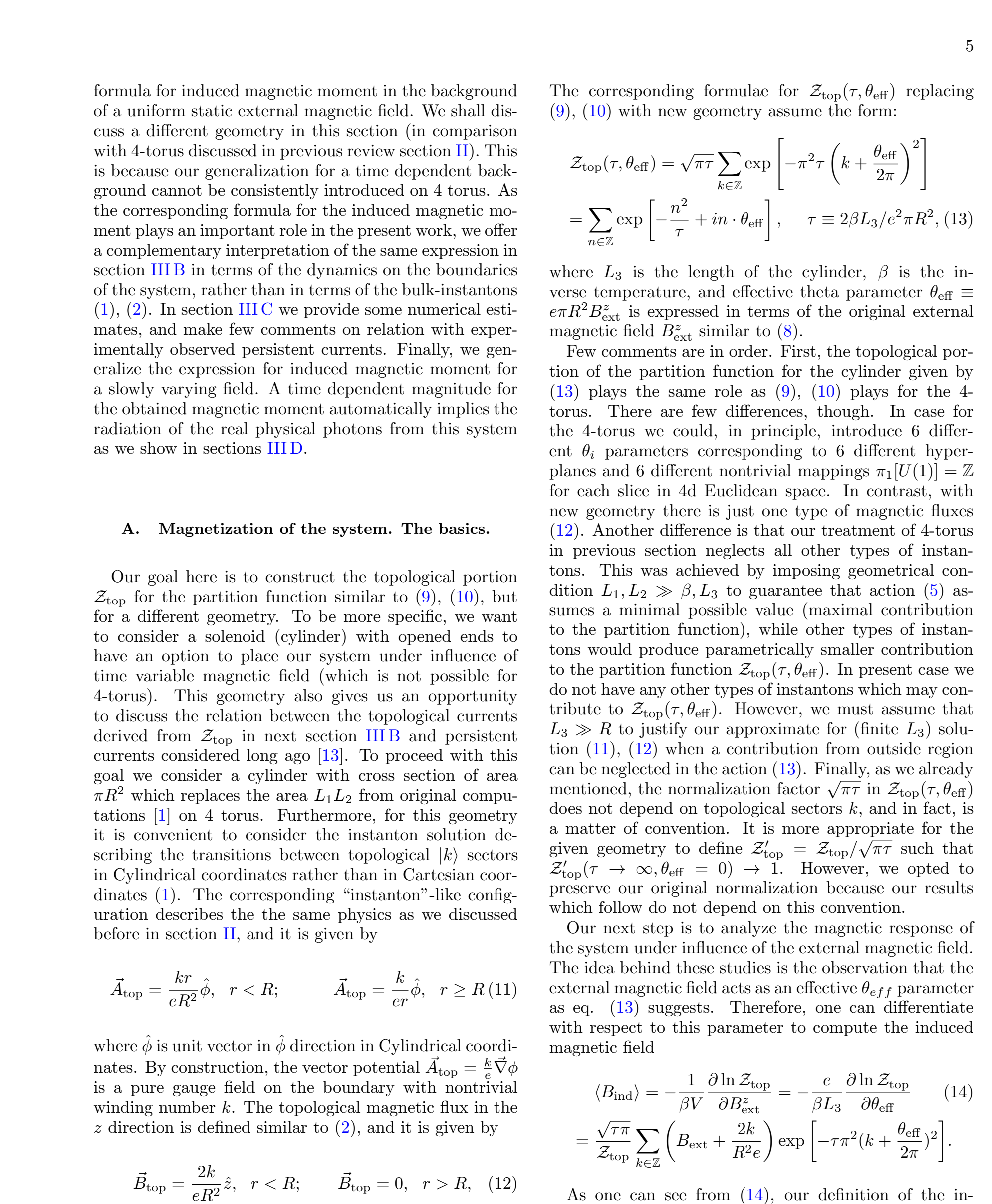

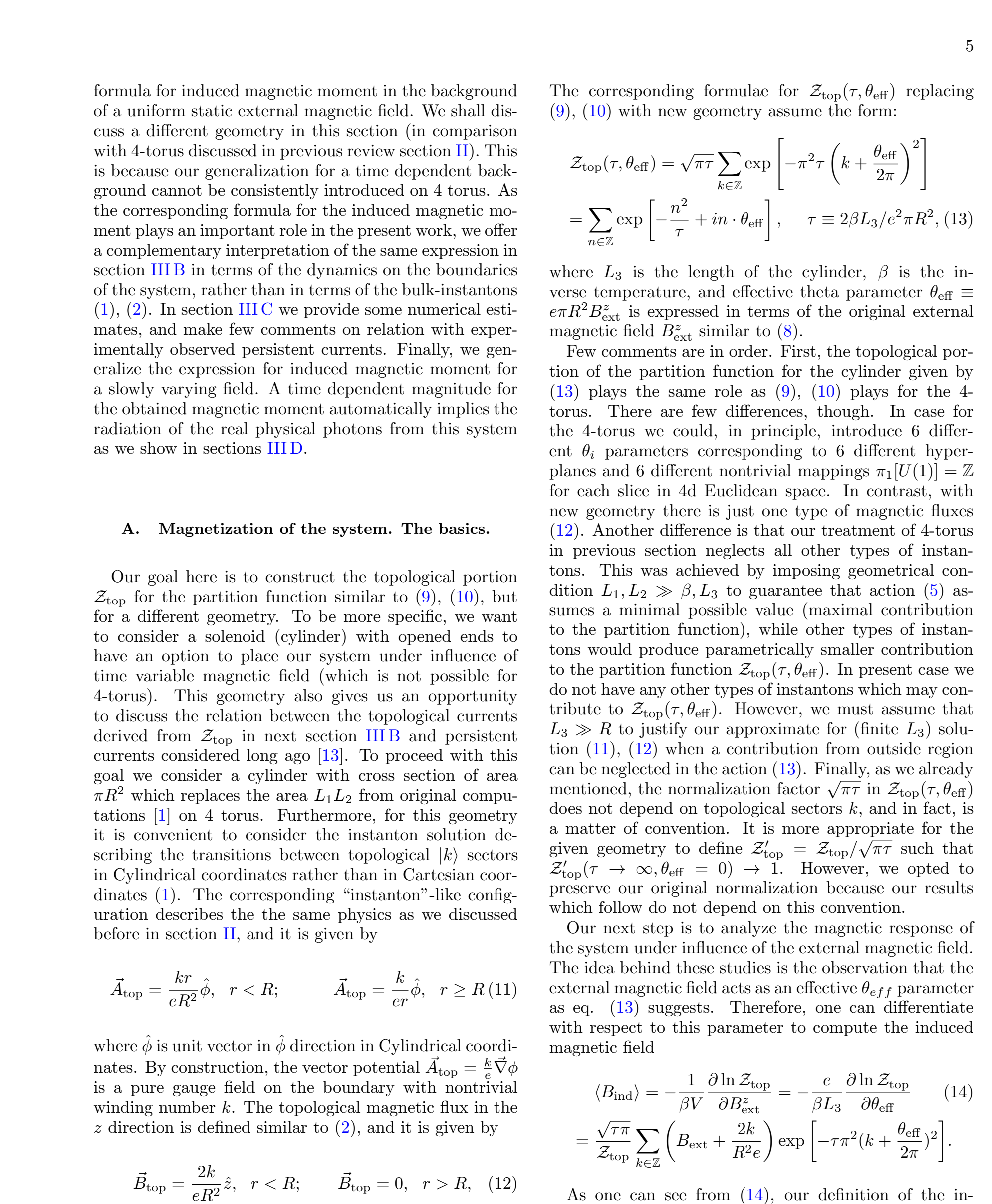

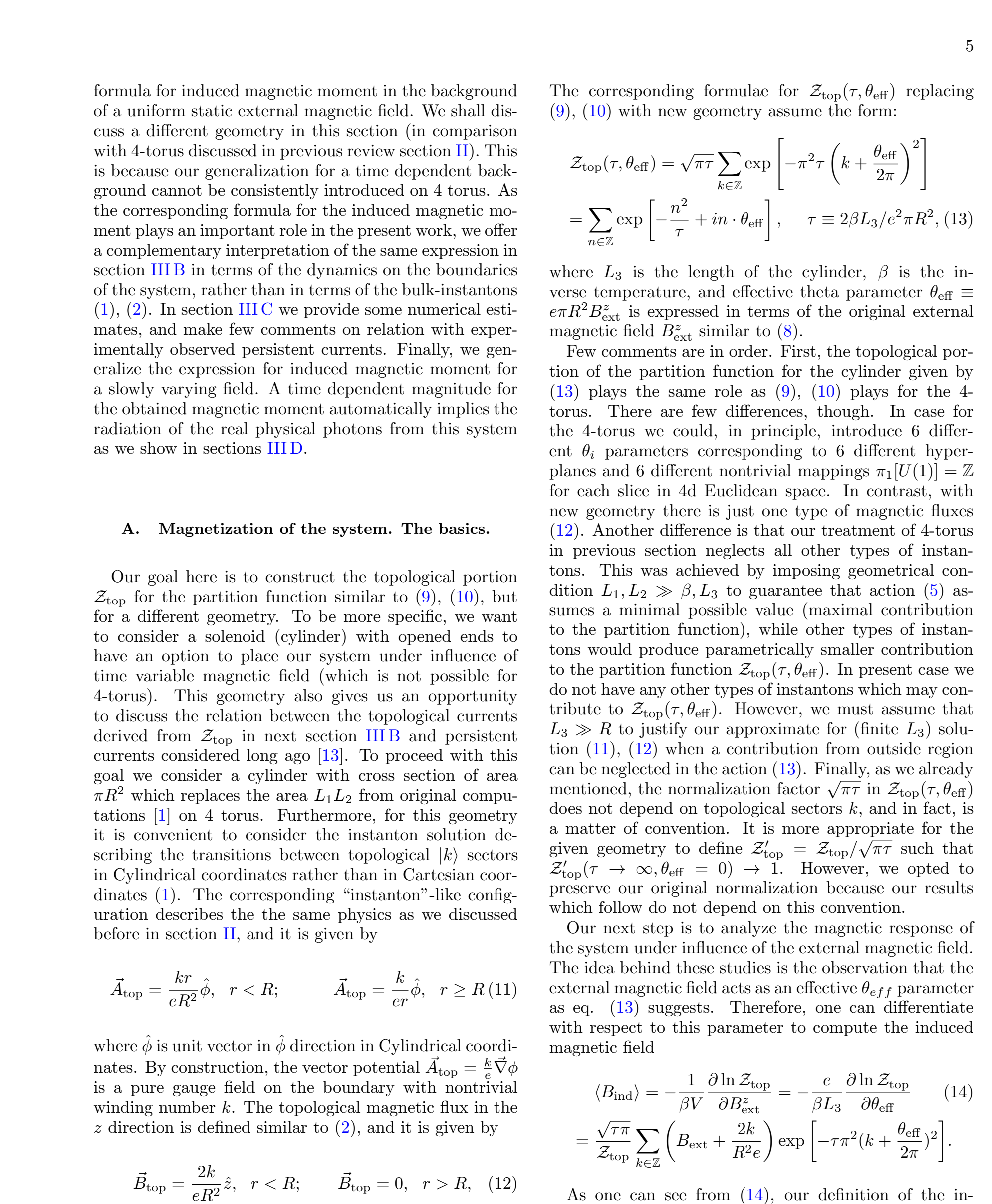

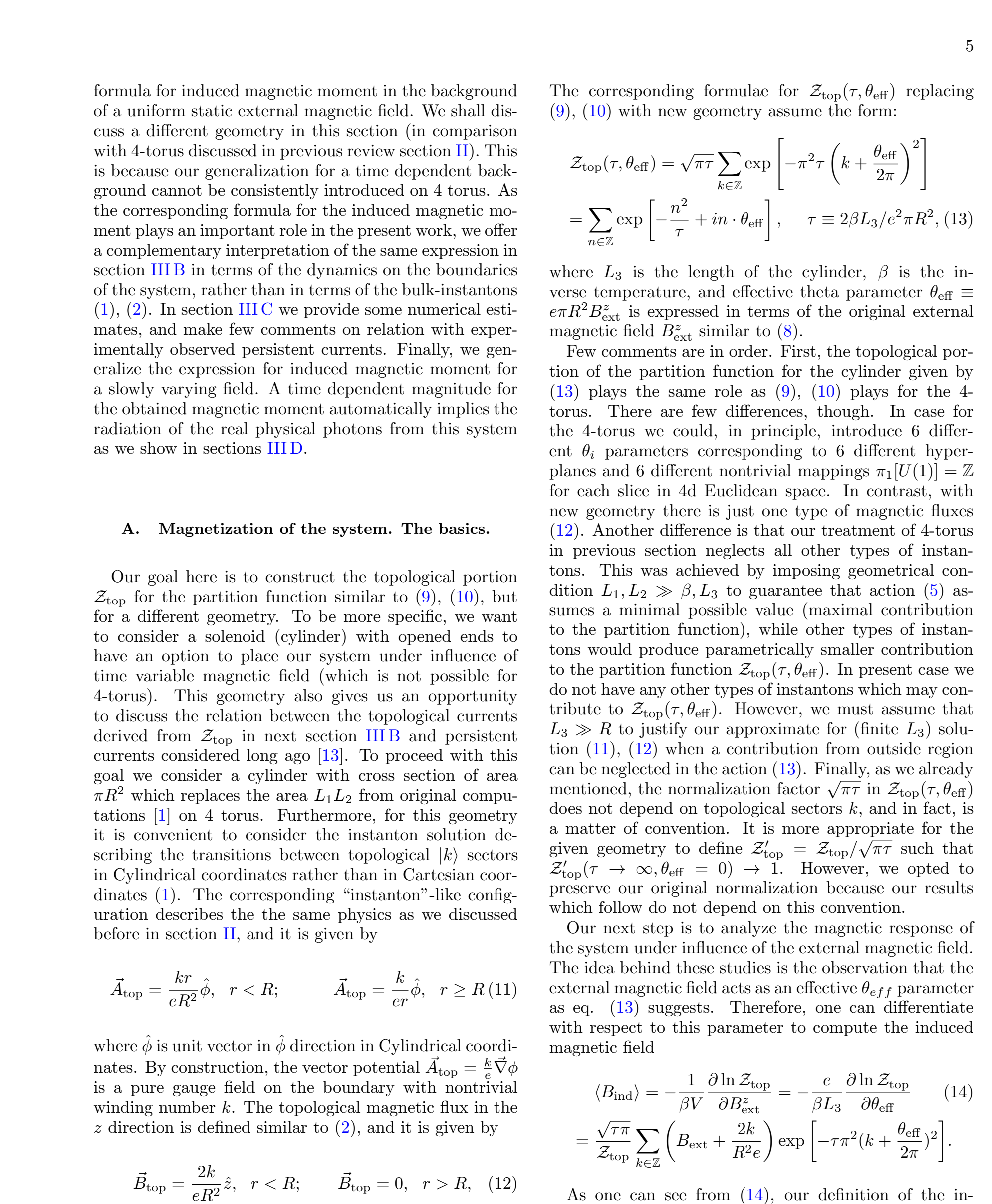

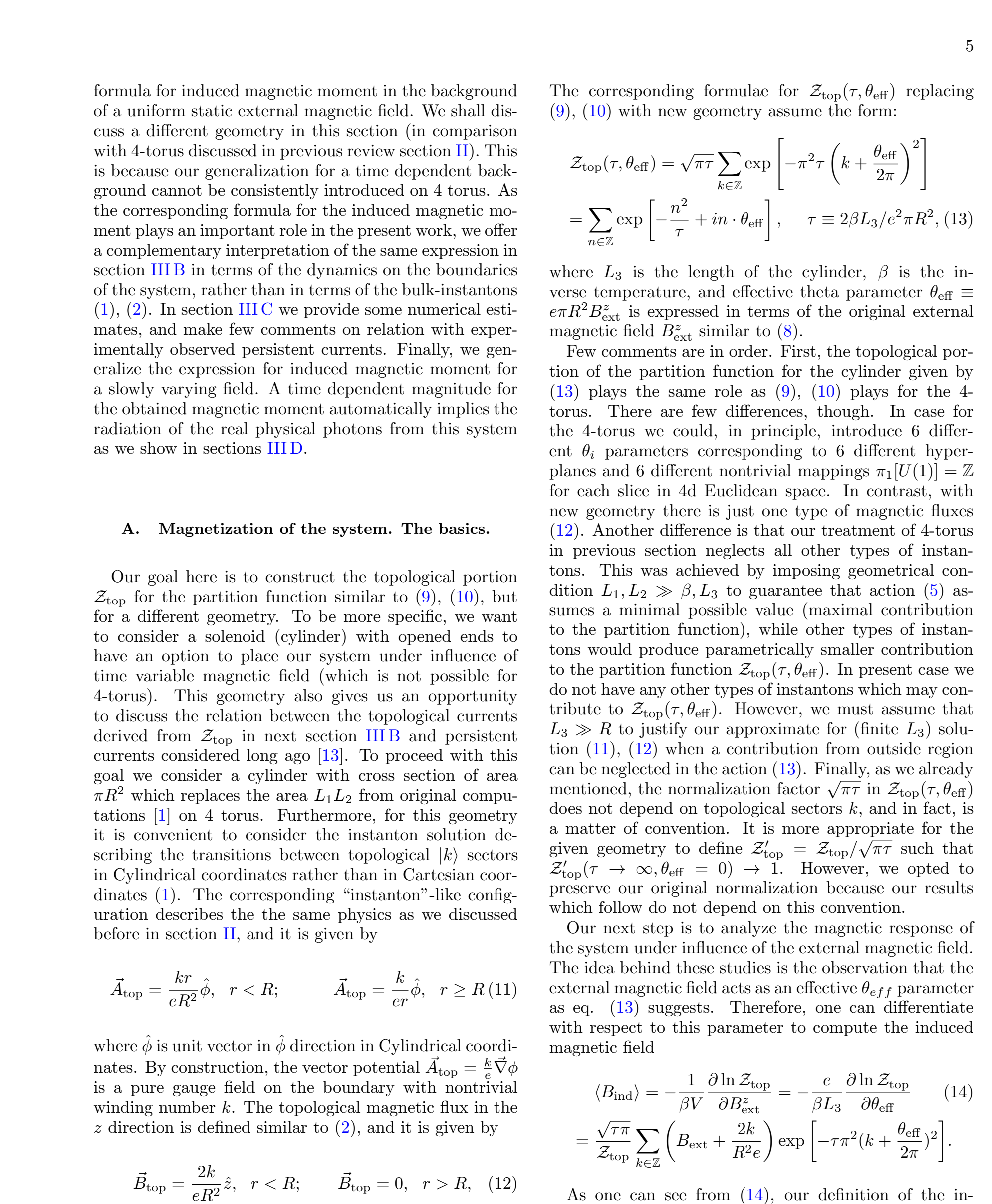

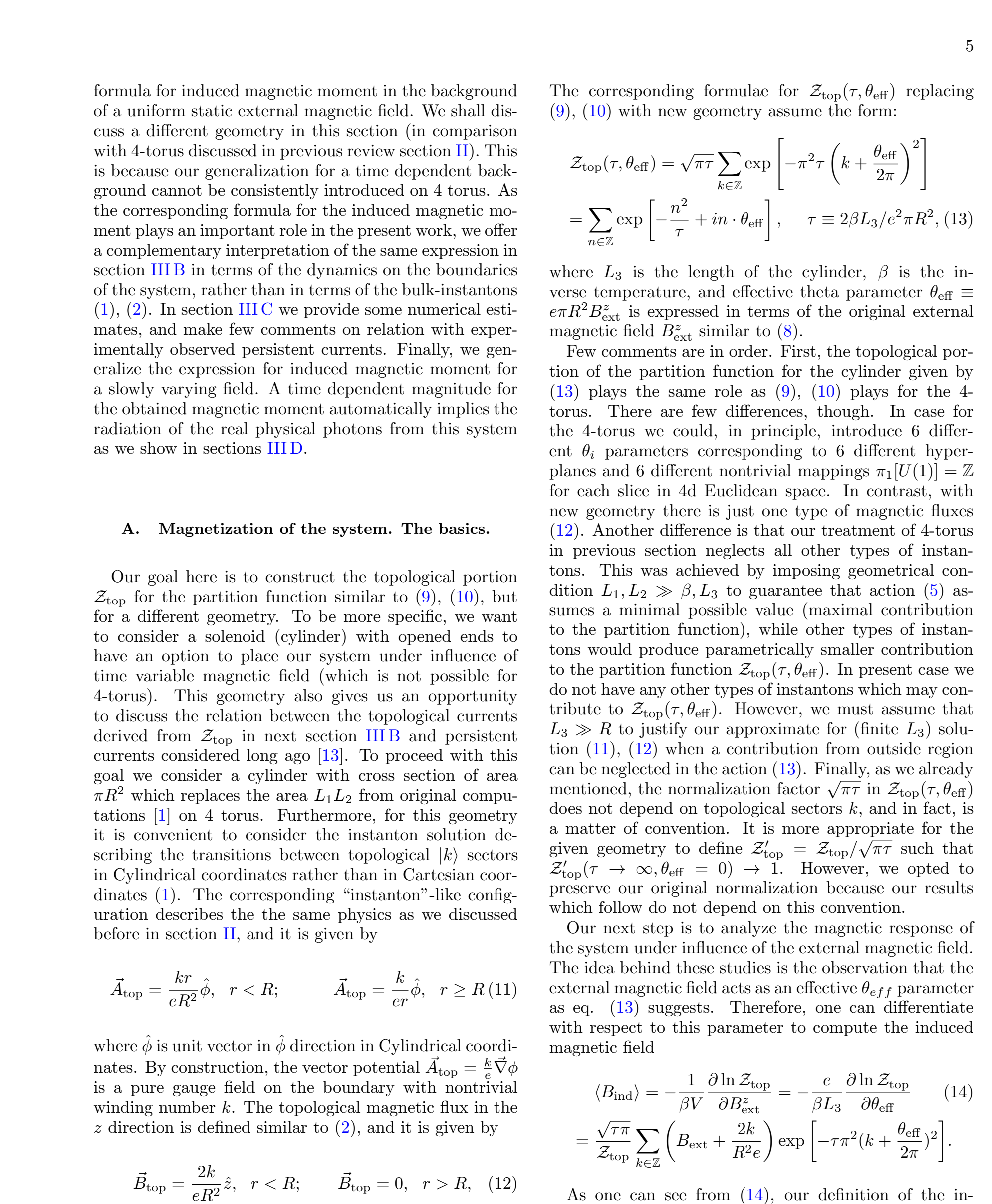

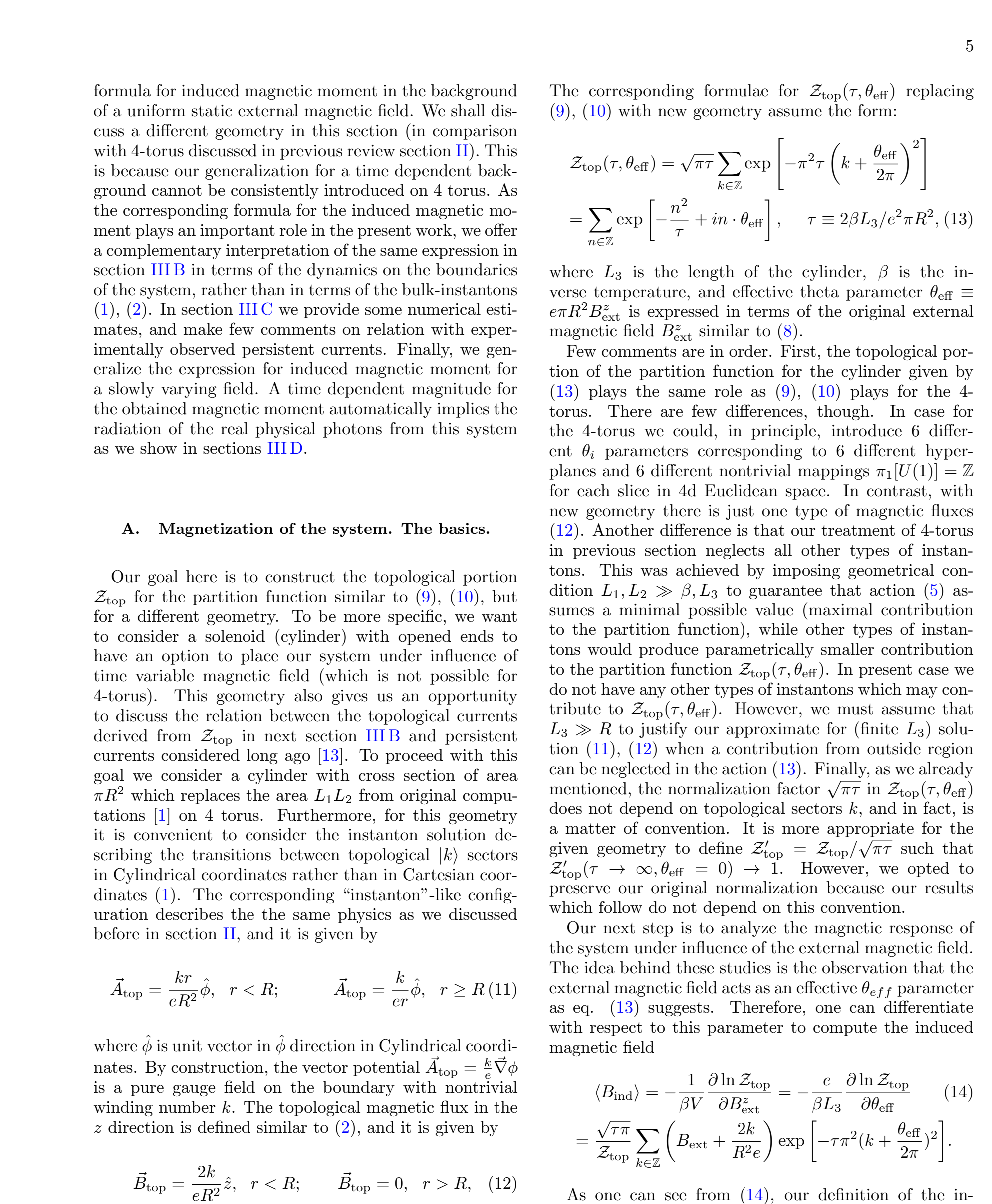

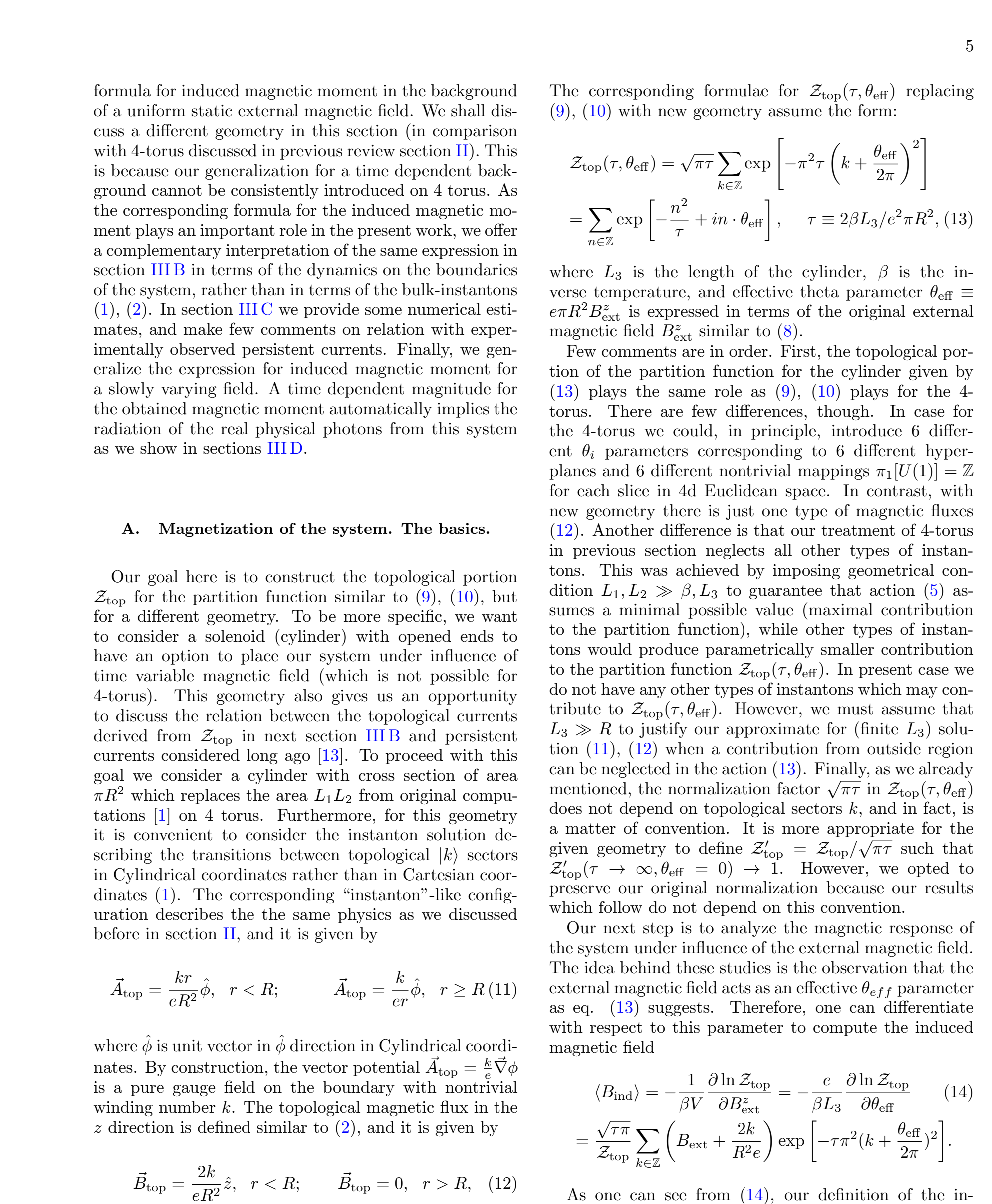

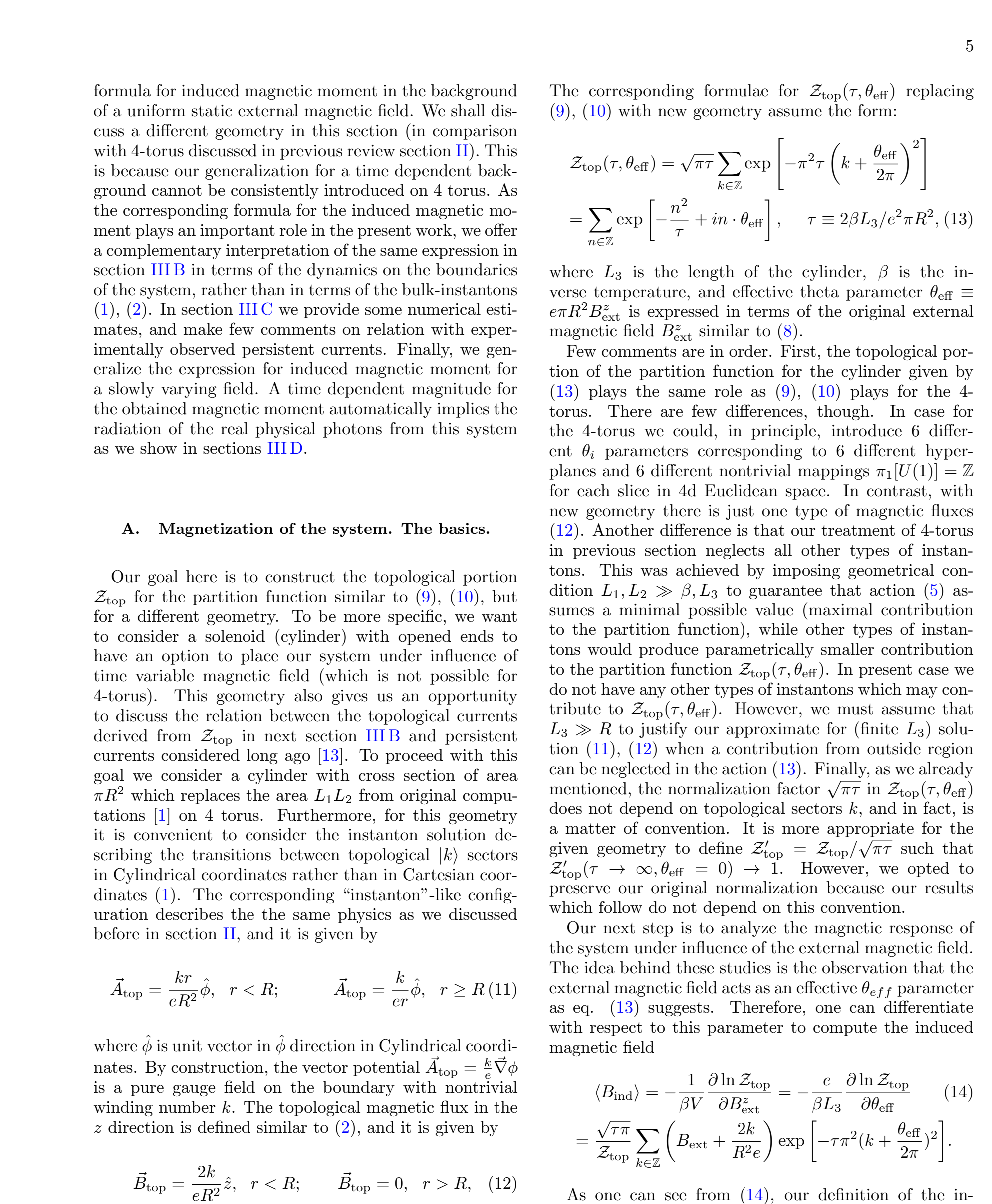

$$
\begin{gathered}
\left\langle B_{\text {ind }}\right\rangle=-\frac{1}{\beta V} \frac{\partial \ln \mathcal{Z}_{\text {top }}}{\partial B_{\text {ext }}^{z}}=-\frac{e}{\beta L_{3}} \frac{\partial \ln \mathcal{Z}_{\text {top }}}{\partial \theta_{\text {eff }}} \\
=\frac{\sqrt{\tau \pi}}{\mathcal{Z}_{\text {top }}} \sum_{k \in \mathbb{Z}}\left(B_{\text {ext }}+\frac{2 k}{R^{2} e}\right) \exp \left[-\tau \pi^{2}\left(k+\frac{\theta_{\text {eff }}}{2 \pi}\right)^{2}\right] .
\end{gathered}
$$


portion of the field. In the absence of the external field $\left(B^{\text {ext }}=0\right)$, the series is antisymmetric under $k \rightarrow-k$ and $\left\langle B_{\text {ind }}\right\rangle$ vanishes. It is similar to the vanishing expectation value of the topological density in gauge theories when $\theta=0$. One could anticipate this result from symmetry arguments as the theory must respect $\mathcal{P}$ and $\mathcal{C P}$ invariance at $\theta=0$.

The expectation value of the induced magnetic field exhibits the $2 \pi$ periodicity from the partition function and it reduces to triviality whenever the amount of skewing results in an antisymmetric summation, i.e. $\left\langle B_{\text {ind }}\right\rangle=0$ for $\theta_{\text {eff }} \in\{2 n \pi: n \in \mathbb{Z}\}$. The point $\theta_{\text {eff }}=\pi$ deserves special attention as this point corresponds to the degeneracy, see [2] with detail discussions. This degeneracy can not be detected by an expectation value of any local operator, but rather is classified by a nonlocal operator, similar to studies of the topological insulators at $\theta=\pi$.

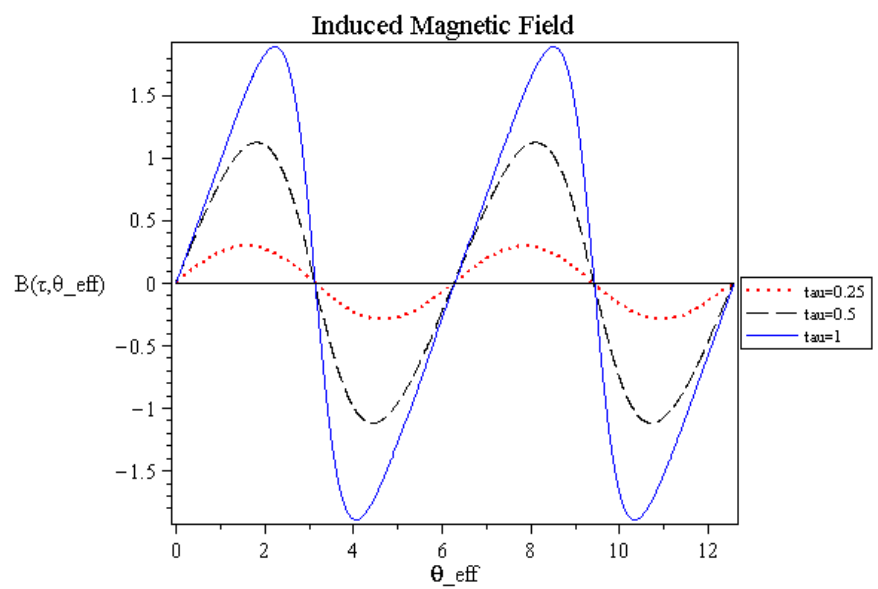

FIG. 1. A numerical plot of the induced magnetic field in units $\frac{c}{\pi R^{2} e}$ as a function of external flux $\theta_{\text {eff }}$. The same plot represents the induced magnetic moment $-\left\langle m_{\text {ind }}^{z}\right\rangle$ in units $\frac{e L_{3} c}{4 \pi \alpha}$, see text with details. Unit magnetic flux corresponds to $\theta_{\text {eff }}=2 \pi$. The plot is adapted from [1].

We now return to analysis of eq. (14). The topological effects, as expected, are exponentially suppressed at $\tau \ll 1$ and $\tau \gg 1$ according to eq.(13). The effect is much more pronounced in the range where $\tau \simeq 1$, see Figure 1, where we plot the induced magnetic field in units $\left(\pi R^{2} e\right)^{-1}$ as a function of external flux $\theta_{\text {eff }}$ (point $\theta_{\text {eff }}=\pi$ should be considered separately as we mentioned above).

Important comment here is that the induced magnetic field defined as (14) can be thought of as the magnetization of the system per unit volume, i.e. $\langle M\rangle=-\left\langle B_{\text {ind }}\right\rangle$, as the definition for $\langle M\rangle$ is identical to (14) up to a minus sign because it enters the Hamiltonian as $H=$ $-\vec{m}_{\text {ind }} \cdot \vec{B}_{\text {ext }}$. Therefore, we arrive to the following expression for the induce magnetic moment of the system in the presence of external magnetic field $B_{\mathrm{ext}}^{z}$,

$$
\begin{gathered}
\left\langle m_{\text {ind }}^{z}\right\rangle=\frac{1}{\beta} \frac{\partial \ln \mathcal{Z}_{\text {top }}}{\partial B_{z}^{\text {ext }}}=-\left\langle B_{\text {ind }}\right\rangle L_{3} \pi R^{2} \\
=-\frac{L_{3} 2 \pi}{e} \frac{\sqrt{\tau \pi}}{\mathcal{Z}_{\text {top }}} \sum_{k \in \mathbb{Z}}\left(\frac{\theta_{\text {eff }}}{2 \pi}+k\right) \exp \left[-\tau \pi^{2}\left(k+\frac{\theta_{\text {eff }}}{2 \pi}\right)^{2}\right] .
\end{gathered}
$$

One can view Fig. 1 as a plot for the induced magnetic moment in units $\frac{e L_{3} c}{4 \pi \alpha}$ which represents correct dimensionality $\frac{\mathrm{e} \cdot \mathrm{cm}^{2}}{\mathrm{~s}}$.

\section{B. Interpretation}

As formula (15) plays a key role in our discussions, we would like to interpret the same expression for $\left\langle m_{\text {ind }}^{z}\right\rangle$ but in different terms. To be more precise: equation (15) describes the magnetization properties of the system in terms of the vacuum configurations describing the tunnelling transitions between topological sectors $|k\rangle$. The corresponding partition function $\mathcal{Z}_{\text {top }}\left(\tau, \theta_{\text {eff }}\right)$ which governs these vacuum processes is given by (13). A nontrivial behaviour of the magnetization of the system in terms of the induced magnetic dipole moment (15) is direct consequence of the basic properties of the partition function $\mathcal{Z}_{\text {top }}\left(\tau, \theta_{\text {eff }}\right)$. We would like to understand the same properties in more intuitive way in terms of the fluctuating currents which unavoidably will be generated on the boundaries, as we discuss below.

Indeed, the cross term in the effective action (8) which describes coupling of the external field with topological instanton-like configuration can be represented as follows

$$
\int \mathrm{d}^{4} x\left(\vec{B}_{\text {ext }} \cdot \vec{B}_{\text {top }}\right)=\int \mathrm{d}^{4} x \vec{A}_{\text {ext }} \cdot\left(\vec{\nabla} \times \vec{B}_{\text {top }}\right),
$$

where we neglected a total divergence term. The cross term written in the form (16) strongly suggests that $\left(\vec{\nabla} \times \vec{B}_{\text {top }}\right)$ can be interpreted as a steady current flow along the boundary. Indeed,

$$
\vec{j}_{\text {top }}(k)=-\vec{\nabla} \times \vec{B}_{\text {top }}=-\delta(r-R) \frac{2 k}{e R^{2}} \hat{\phi},
$$

where we used expression (13) for $\vec{B}_{\text {top }}$ describing the tunnelling transition to $|k\rangle$ sector. Formula (17) is very suggestive and implies that the vacuum transitions formulated in terms of the fluxes-instantons (11), (12) can be also interpreted in terms of accompanied fluctuating topological currents (17). The total current (in topological $k$ sector) which flows along the infinitely thin boundary of a cylinder radius $R$ and length $L_{3}$ in our ideal system is given by

$$
J^{\phi}(k)=\int_{0}^{L_{3}} d z \int d r j_{\text {top }}^{\phi}=-\frac{2 k L_{3}}{e R^{2}} .
$$

This current in topological $k$ sector produces the following contribution to the magnetic dipole moment:

$$
m_{\text {ind }}^{z}(k)=\pi R^{2} J^{\phi}(k)=-\frac{2 \pi L_{3}}{e} k .
$$


This formula precisely reproduces the term proportional to $k$ in the parentheses in eq. (15) which was originally derived quite differently, see previous subsection III A.

Few comments on eq. (18). The expectation value $\left\langle J^{\phi}\right\rangle$ of the topological current obviously vanishes at zero external magnetic field when one sums over all topological $k$-sectors, in agreement with our previous expression (15) with $\theta_{\text {eff }}=0$. It is quite obvious that the topological currents have pure quantum nature as they effectively represent the instantons describing the tunnelling transitions in the path integral computations. The currents could have clockwise or anticlockwise direction, depending on sign of integer number $k$, similar to fluctuating instanton solutions (11), saturating the topological portion of the partition function (13).

Furthermore, one can explicitly check that the cross term (16) computed in terms of the boundary current $\vec{j}_{\text {top }}(k)$ exactly reproduces the corresponding term in the action for the partition function (15) computed in terms of the bulk instantons (11), (13). Indeed,

$$
\begin{gathered}
\int d^{4} x \vec{A}_{\mathrm{ext}} \cdot\left(\vec{\nabla} \times \vec{B}_{\mathrm{top}}\right)=\frac{2 k\left(2 \pi \beta L_{3}\right)}{e R^{2}} \int r d r \delta(r-R) A_{\mathrm{ext}}^{\phi} \\
=\frac{2 k\left(2 \pi \beta L_{3}\right)}{e R^{2}} \cdot\left(\frac{B_{\mathrm{ext}}^{z} R^{2}}{2}\right)=2 \tau \pi^{2} k\left(\frac{\theta_{\mathrm{eff}}}{2 \pi}\right),
\end{gathered}
$$

where the vector potential $A_{\text {ext }}^{\phi}(r)=\frac{r}{2} B_{\text {ext }}^{z}$ corresponds to the external uniform magnetic field in cylindrical coordinates. Our final result in eq. (20) is expressed in terms of the external flux $\theta_{\text {eff }} \equiv e \pi R^{2} B_{\text {ext }}^{z}$ and dimensionless parameter $\tau \equiv 2 \beta L_{3} / e^{2} \pi R^{2}$. One can explicitly see that the cross term in action in eq. (15) is reproduced by eq. (20) derived in terms of the boundary currents, rather then in terms of the bulk instantons.

The classical instanton action is also reproduced in terms of the boundary currents. Indeed, by substituting the expression for the current (17) to the classical action one arrives to

$$
\begin{aligned}
& \frac{1}{2} \int \mathrm{d}^{4} x\left(\vec{B}_{\mathrm{top}} \cdot \vec{B}_{\mathrm{top}}\right)=\frac{1}{2} \int \mathrm{d}^{4} x \vec{A}_{\mathrm{top}} \cdot\left(\vec{\nabla} \times \vec{B}_{\mathrm{top}}\right), \\
= & \frac{k\left(2 \pi \beta L_{3}\right)}{e R^{2}} \int r d r \delta(r-R) A_{\mathrm{top}}^{\phi}(r)=\tau \pi^{2} k^{2},
\end{aligned}
$$

which is precisely the expression for classical instanton action entering the topological portion of the partition function (15).

The basic point of our discussions in this section is that the expression for the induced magnetic moment (15) can be understood in terms of the topological currents flowing along the boundaries of the system. However, the origin of the phenomena is not these currents but the presence of the topological $|k\rangle$ sectors in Maxwell $U(1)$ electrodynamics when it is formulated on a compact manifold with nontrivial mapping $\pi_{1}[U(1)]=\mathbb{Z}$. Such $|k\rangle$ sectors exist and transitions between them always occur even if charged particles are not present in the system. The coupling of the non-trivial gauge configurations describing the transitions between the $|k\rangle$ sectors with charged particles in the presence of external magnetic field leads to such pronounced effect as persistent currents $[13,14]$. The secondary, rather than fundamental role of the charged matter particles in this phenomenon manifests, in particular, in a fact that $\mathcal{Z}_{\text {top }}$ generates an extra contribution to the Casimir vacuum pressure even at zero external magnetic field. At the same time the persistent current can not be generated at $B_{\text {ext }}=0$ as clockwise and counterclockwise currents cancel each other.

Furthermore, the persistent currents in the original works $[13,14]$ were introduced as a response of the electrons (residing on the ring) on external magnetic flux with nontrivial Aharonov -Bohm phase. In contrast, the non-trivial gauge configurations (and accompanied topological currents (17) in $k$ sectors) in our system are generated even when no external field nor corresponding external Aharonov Bohm vector potential are present in the system. In addition, the correlation length in conventional persistent currents $[13,14]$ is determined by dynamics of the electrons residing on the ring, while in our case it is determined by the dynamics of the vacuum described by the partition function $\mathcal{Z}_{\text {top }}$. The corresponding topological fluctuations (described in terms of the instantons (13)) also generate the persistent topological currents on the boundary (17). However, this additional contribution should be treated separately from conventional persistent currents [13, 14], as it is absolutely independent contribution which is generated due to the unavoidable coupling of topological gauge configurations with charged particles on the boundary of the system $^{1}$.

Finally, the induced magnetic moment (19) due to the topological currents flowing on the boundary is quantized. Indeed, $m_{\text {ind }}^{z} / L_{3}$ assumes only integer numbers in units of $\frac{2 \pi}{e}$. This is because the corresponding induced currents always accompany the quantized instanton -like fluctuations (12). In contrast, a similar induced magnetic moment due to the conventional persistent currents $[13,14]$ is not quantized, and can assume any value.

To conclude this subsection we would like to comment that it is quite typical in condensed matter physics that the topologically ordered systems exhibit such a com-

\footnotetext{
1 In many respects this situation is very similar to QCD when the presence of the topological sectors is absolutely fundamental basic property of the gauge system. At the same time, very pronounced consequences of this fundamental feature are expressed in terms of the matter fermi fields, rather than in terms of original gauge configurations. These well noticeable properties of the system are basically the consequence of the Index Theorem which states that the fermions in the background of nontrivial gauge configurations have chiral zero modes. Precisely these zero modes play extremely important role in explanation of many effects such as generation of the chiral condensate in QCD, the resolution of the so-called the $U(1)_{A}$ problem, etc. However, the root, the origin of these properties of the system is the presence of topologically non-trivial gauge configurations, while the matter fields play the secondary role.
} 
plementary formulation in terms of the physics on the boundary. Our system $(\mathcal{T V})$ can be also thought as a topologically ordered system as argued in $[2,3]$ because it demonstrates a number of specific features which are inherent properties of topologically ordered systems. In particular, $\mathcal{Z}_{\text {top }}\left(\tau, \theta_{\text {eff }}\right)$ demonstrates the degeneracy of the system which can not be described in terms of any local operators. Furthermore, the infrared physics of the system can be studied in terms of auxiliary topological non-propagating fields precisely in the same way as a topologically ordered system in condensed matter physics can be analyzed in terms of the Berry's connection. Therefore, it is not a surprise that we can reformulate the original instanton fluctuations saturating $\mathcal{Z}_{\text {top }}\left(\tau, \theta_{\text {eff }}\right)$ in terms of the boundary persistent currents which always accompany these instanton transitions.

\section{Numerical estimates}

We want to make some simple numerical estimates by comparing the magnetic induced moment $\left\langle m_{\text {ind }}^{z}\right\rangle$ from eq. (15) with corresponding expression $m_{\text {persistent }} \simeq \pi I_{0} R^{2}$ with measured persistent current $I_{0}$. One should emphasize that the conventional persistent currents are highly sensitive to the properties of the material. More than that, the properties of the condensed matter samples essentially determine the magnitude of the measured currents. At the same time, in all our discussions above we assume that the "ideal" boundary conditions can be arranged, such that the Maxwell vacuum defined on a compact manifold is well described by the partition function (9), (10). Moreover, as we discussed in previous section III B the topological boundary currents (17) in our framework should be considered as an independent additional contribution to conventional persistent currents. Therefore, the corresponding numerical estimates taken from early work [15] are presented here for demonstration purposes only.

The measurement of the typical persistent current was reported in [15]. The measurements were performed on single gold rings with diameter $2 R=2.4$ and $4 \mu \mathrm{m}$ at a base temperature of $4.5 \mathrm{mK}$. Reported values for the currents are $I_{0}=3$ and $30 \mathrm{nA}$ for these two rings. It should be contrasted with expected current $\sim 0.1 \mathrm{nA}$. We are not in position to comment on this discrepancy, as the effect is basically determined by the properties of the material, which is not subject of the present work. Our goal is in fact quite different. We want to compare the magnetic moment which is induced due to this persistent current with induced magnetic moment due to the topological vacuum configurations $\left\langle m_{\text {ind }}^{z}\right\rangle$ from eq. (15). Numerically, a magnetic moment for the largest observed current (30 nA) can be estimated as follows

$$
\begin{aligned}
m_{\text {persistent }} & \simeq \pi I_{0} R^{2} \sim \pi(30 \mathrm{nA}) \cdot\left(\frac{2.4 \mu \mathrm{m}}{2}\right)^{2} \\
& \sim 0.7 \cdot 10^{4}\left(\frac{e \mathrm{~cm}^{2}}{\mathrm{~s}}\right) .
\end{aligned}
$$

It is instructive to compare this moment with fundamental Bohr magneton $\mu_{B}=e \hbar / 2 m_{e} \simeq 0.6 \cdot\left(\frac{e \mathrm{~cm}^{2}}{\mathrm{~s}}\right)$, which provides a crude estimate of a number of effective degrees of freedom $\sim 10^{4}$ which generate the persistent current $I_{0}$ for this specific sample. This estimate should be taken with some precaution because the effect of persistent currents is entirely determined by the properties of the material (such as the electron phase coherence length $l_{\phi}$ ) which is beyond of the scope of the present work.

Before we estimate $\left\langle m_{\text {ind }}^{z}\right\rangle$ from eq. (15) to compare it with (22) we would like to get some insights about the numerical magnitude of the dimensionless parameter $\tau$ for the ring with parameters used in the estimate (22),

$$
\tau \equiv \frac{2 \beta L_{3}}{e^{2} \pi R^{2}} \sim \frac{2(0.1 \mu \mathrm{m})(0.6 \mathrm{~cm})}{4 \pi^{2} \alpha}\left(\frac{2}{2.4 \mu \mathrm{m}}\right)^{2} \gg 1,
$$

where we use for $L_{3} \sim 0.1 \mu \mathrm{m}$ and $\beta \sim 0.6 \mathrm{~cm}$ which corresponds to the temperature $T \simeq 300 \mathrm{mK}$ below which $l_{\phi}$ is sufficiently large and temperature independent ${ }^{2}$. Large magnitude of $\tau$ implies that for the chosen parameters for the system the vacuum transitions between the topological sectors are strongly suppressed as the expression for the partition function (9), (10) states. In this regime the effect (22) is entirely determined by conventional mechanism [13], [14].

If somehow we could manage to satisfy our ideal boundary conditions and could adjust parameters of the system such that $\tau \sim 1$ than the magnitude of $\left\langle m_{\text {ind }}^{z}\right\rangle$ from eq. (15) is determined by parameter $2 \pi L_{3} / e$ such that

$$
\left\langle m_{\mathrm{ind}}^{z}\right\rangle \sim \frac{2 \pi L_{3}}{e} \sim \frac{L_{3} c e}{2 \alpha} \sim 1.5 \cdot 10^{7}\left(\frac{e \mathrm{~cm}^{2}}{\mathrm{~s}}\right),
$$

which is at least 3 orders of magnitude larger than the value (22) of the magnetic moment generated due to conventional persistent current when the correlation length is determined by the physics of the ring. The crucial element in our estimate (24) is that the key parameter $\tau$ should be order of one, $\tau \sim 1$. This would guarantee that the vacuum transitions would not be strongly suppressed. We really do not know if it could be realized in practice. The answer hopefully could be positive as Aharonov Bohm phase coherence can be maintained at sufficient high temperature, which can drastically decrease parameter $\tau$ from (23), see footnote 2 .

\footnotetext{
2 One should remark here that there are related effects when the entire system can maintain the Aharonov Bohm phase coherence at very high temperature $T \simeq 79 \mathrm{~K}[16]$.
} 
We emphasize once again that eq. (24) describes a new contribution to the magnetic moment originated from tunnelling transitions between topological $k$ sectors. It should be contrasted with conventional persistent current which also contributes to magnetic moment (22). These two contributions originated form very different physics: in case eq. (22) the correlation in the system is achieved by the dynamics of the electrons on the boundary, while in our case it is achieved by the tunnelling transitions between gauge $k$ sectors and described by the vacuum instantons (13) saturating $\mathcal{Z}_{\text {top }}\left(\tau, \theta_{\text {eff }}\right)$.

\section{E\&M Radiation}

Important comment we would like to make is as follows. Formula (15) has been derived assuming that the external field is static. However, formula (15) still holds even in the case when the time dependence is adiabatically slow, i.e. $\left(\frac{d B_{\text {ind }}}{d t}\right) / B_{\text {ind }}=\omega$ is much smaller than any relevant scales of the problem, to be discussed below. Therefore, one can use the well -known expressions for the intensity $\vec{S}$ and total radiated power $I$ for the magnetic dipole radiation when dipole moment (15) varies with time:

$$
\vec{S}=I(t) \frac{\sin ^{2} \theta}{4 \pi r^{2}} \vec{n}, \quad I(t)=\frac{2}{3 c^{2}}\left\langle\ddot{m}_{\text {ind }}^{z}\right\rangle^{2}
$$

In case when the external magnetic field in the vicinity of $\theta_{\text {eff }} \sim 2 \pi n$ the behaviour of the induced magnetic moment almost linearly follows $B_{\text {ext }}^{z}(t)$ as one can see from Figure 1. In particular, if $B_{\text {ext }}^{z}(t) \sim \cos \omega t$ than $\left\langle\ddot{m}_{\text {ind }}^{z}\right\rangle \sim \omega^{2} \cos \omega t$. In this case one can easily compute the average intensity over large number of complete cycles with the result

$$
\langle I\rangle \sim \frac{\omega^{4}}{3 c^{2}}\left\langle m_{\mathrm{ind}}^{z}\right\rangle^{2},
$$

where $\left\langle m_{\text {ind }}^{z}\right\rangle$ is given by (15).

Few comments are in order. First of all, the magnetic dipole radiation can be easily understood in terms of persistent currents $[13,14]$ flowing along the ring. For static external magnetic field the corresponding persistent current $I_{0}$ is also time independent. The magnetic dipole moment generated by this current can be estimated as $m_{\text {persistent }} \simeq \pi I_{0} R^{2}$. When the external magnetic field starts to fluctuate, the corresponding current $I_{0}(t)$ as well as magnetic dipole moment $m_{\text {persistent }}(t)$ also become time-dependent functions. It obviously leads to the radiation of real photons which is consistent with our analysis. However, we should emphasize that the interpretation of this phenomenon (which we coin as nonstationary TCE) in terms of topological persistent currents (17) is the consequential, rather than fundamental explanation. The fundamental explanation, as emphasized in the previous section III B is based on topological instanton-like configurations interpolating between $k$ topological sectors. These tunnelling transitions occur in the system even when persistent currents are not generated in the system (for example in absence of external field).

Our final comment in this subsection is as follows. As we discussed above the energy for $E \& M$ radiation eventually comes from time-dependent external magnetic field. One could suspect that it would be very difficult to discriminate a (non-interesting) direct emission originated from $B_{\text {ext }}(t) \neq 0$ and the (very interesting) emission resulted from the Maxwell vacuum which itself is excited due to the quantum interference of the vacuum configurations describing the topological $|k\rangle$ sectors with external magnetic field. First (non-interesting) term is represented by $\theta_{\text {eff }}=e B_{\text {ext }}(t) \pi R^{2}$ in the parentheses in eq. (15), while the second (very interesting) term is represented by term $\sim k$ in eq. (15).

Fortunately, one can easily discriminate between (the very interesting) emission from the vacuum and (absolutely non- interesting) background radiation. The point is that the induced magnetic dipole moment $\left\langle m_{\text {ind }}^{z}(t)\right\rangle$ is the periodic function of $B_{\text {ext }}(t)$. Exactly at the point $\theta_{\text {eff }}=\pi$ the induced magnetic dipole moment $\left\langle m_{\text {ind }}^{z}\right\rangle$ suddenly changes the sign as one can see from Figure 1. This is a result of complete reconstruction of the ground state in the vicinity of $\theta_{\text {eff }}=\pi$ when the level crossing occurs, which eventually results in the double degeneracy of the system at this point. As this is the key element of the construction which leads to the important observational consequences related to the topological features of the system, we elaborate on this issue with more details in Appendix A.

Therefore, one could slowly change the external field $B_{\text {ext }}(t)$ in vicinity of $\theta_{\text {eff }}=\pi$ which corresponds to the half integer flux, to observe the variation in intensity and polarization of radiation. The corresponding background radiation must vary smoothly, while the emission from vacuum should change drastically. One could hope that these drastic changes may serve as a smoking gun for discovery of a fundamentally novel type of radiation from topological Maxwell vacuum, similar to DCE.

\section{CONCLUSION AND FUTURE DIRECTIONS}

In this work we discussed a number of very unusual features exhibited by the Maxwell theory formulated on a compact manifold $\mathbb{M}$ with nontrivial topological mapping $\pi_{1}[U(1)]$, which was coined the topological vacuum $(\mathcal{T V})$. All these features are originated from the topological portion of the partition function $\mathcal{Z}_{\text {top }}\left(\tau, \theta_{\text {eff }}\right)$ and can not be formulated in terms of conventional $E \& M$ propagating photons with two physical transverse polarizations. In different words, all effects discussed in this paper have a non-dispersive nature.

The computations of the present work along with previous calculations of refs. [1-3] imply that the extra energy (and entropy), not associated with any physical 
propagating degrees of freedom, may emerge in the gauge systems if some conditions are met. This fundamentally new type of energy emerges as a result of dynamics of pure gauge configurations at arbitrary large distances. The new idea advocated in this work is that this new type of energy can be, in principle, studied if one place the system in time-dependent background. In this case we expect that the vacuum topological configurations can radiate conventional photons which can be detected and analyzed.

This unique feature of the system when an extra energy is not related to any physical propagating degrees of freedom was the main motivation for a proposal $[17,18]$ that the vacuum energy of the Universe may have, in fact, precisely such non-dispersive nature ${ }^{3}$. This proposal when an extra energy can not be associated with any propagating particles should be contrasted with a conventional description when an extra vacuum energy in the Universe is always associated with some ad hoc physical propagating degree of freedom, such as inflaton ${ }^{4}$.

Essentially, the proposal $[17,18]$ identifies the observed vacuum energy with the Casimir type energy, which however is originated not from dynamics of the physical propagating degrees of freedom, but rather, from the dynamics of the topological sectors which are always present in gauge systems, and which are highly sensitive to arbitrary large distances. Furthermore, the radiation from the vacuum in a time-dependent background (which is the main subject of this work) is very similar in all respects to the radiation which might be responsible for the end of inflation in that proposal, see [18] for the details. The present study, in fact, is motivated by the cosmological ideas [18] which hopefully can be tested in a tabletop experiment when the vacuum energy in timedependent background can be transferred to real propagating degrees of freedom as suggested in section IIID. In cosmology the corresponding period plays a crucial role and calls the reheating epoch which follows the inflation when the vacuum energy is the dominating component of the Universe.

To conclude, the main point of the present studies is that the radiation may be generated from the vacuum

3 This new type of vacuum energy which can not be expressed in terms of propagating degrees of freedom has been in fact well studied in QCD lattice simulations, see [17] with large number of references on the original lattice results.

${ }^{4}$ There are two instances in evolution of the universe when the vacuum energy plays a crucial role. First instance is identified with the inflationary epoch when the Hubble constant $H$ was almost constant which corresponds to the de Sitter type behaviour $a(t) \sim \exp (H t)$ with exponential growth of the size $a(t)$ of the Universe. The second instance when the vacuum energy plays a dominating role corresponds to the present epoch when the vacuum energy is identified with the so-called dark energy $\rho_{D E}$ which constitutes almost $70 \%$ of the critical density. In the proposal $[17,18]$ the vacuum energy density can be estimated as $\rho_{D E} \sim H \Lambda_{Q C D}^{3} \sim\left(10^{-4} \mathrm{eV}\right)^{4}$, which is amazingly close to the observed value. configurations describing the tunnelling transitions between $|k\rangle$ sectors, rather than from physical propagating degrees of freedom, which would correspond to conventional DCE when the virtual photons become real photons in a time dependent background. This is precisely the difference between DCE and non-stationary TCE considered in the present work.

\section{ACKNOWLEDGEMENTS}

I am thankful to Maxuim Chernodub for discussions and useful comments. I am also thankful to Alexei Kitaev for long and interesting discussions on relation of the persistent currents $[13,14]$ and the topological configurations saturating $\mathcal{Z}_{\text {top }}$. This research was supported in part by the Natural Sciences and Engineering Research Council of Canada.

\section{Appendix A: Classification of the vacuum states, Degeneracy, and the Topological order.}

The main goal of this Appendix is to review and elaborate on important property of degeneracy of the system under study. As suggested in section III D the feature of degeneracy may play an important role in discrimination of novel and interesting effect of emission of real photons from vacuum (as a result of the non-static Topological Casimir Effect) from the background radiation.

The starting point is to analyze the symmetry properties of partition function $\mathcal{Z}_{\text {top }}\left(\tau, \theta_{\text {eff }}\right)$ defined by eq.(13). One can easily observe that the $\theta_{\text {eff }}=\pi$ is very special point as even and odd " $n$ "- terms in the dual representation for $\mathcal{Z}_{\text {top }}\left(\tau, \theta_{\text {eff }}\right)$ contribute equally to the partition function. It obviously leads to the degeneracy of the system at $\theta_{\text {eff }}=\pi$. The conventional, non-topological, part of the partition function $\mathcal{Z}_{\text {quant }}\left(\tau, \theta_{\text {eff }}\right)$ is not sensitive to the topological sectors at all, as discussed in section II A. Therefore, this property of degeneracy is an exact feature of the system. This double degeneracy implies that $\mathbb{Z}_{2}$ symmetry is spontaneously broken.

What is the symmetry which is spontaneously broken? What is order parameter which classifies two physically distinct states? One can not formulate the corresponding symmetry breaking effect in terms of any local operators and their vacuum expectation values as argued in [2]. Rather, a proper classification of the ground state is formulated in terms of non-local operators. Indeed, a corresponding order parameter which characterizes the system is

$$
\begin{aligned}
\left\langle\frac{e}{2 \pi} \oint A_{i} d x_{i}\right\rangle_{\theta_{\text {eff }}=\pi-\epsilon} & =+\frac{1}{2} \\
\left\langle\frac{e}{2 \pi} \oint A_{i} d x_{i}\right\rangle_{\theta_{\text {eff }}=\pi+\epsilon} & =-\frac{1}{2}
\end{aligned}
$$

where computations should be carefully carried out by approaching $\theta_{\text {eff }}=\pi$ in partition function $\mathcal{Z}_{\text {top }}\left(\tau, \theta_{\text {eff }}\right)$ 
from two opposite sides of the $\theta_{\text {eff }}=\pi \pm \epsilon$ as discussed in [2]. This classification is very different from the conventional Landau classification based on broken symmetries when a system is characterized by some expectation value of a local operator. One should note that a similar classification is known to emerge in topologically order systems, e.g. topological insulators at $\theta=\pi$, see recent reviews [5-9]. In fact the classification (A1) is a very particular example of a much more generic framework recently discussed in [19] when the classification of a ground state is based on some global rather than local observables.

We want to emphasize that classification (A1) is not a unique feature of the abelian gauge theory. Similar classification of the ground states also emerges in nonabelian gauge field theories. We would like to mention in this Appendix just one specific example, the so-called "deformed QCD" model [20] because its close relation to real QCD, and most importantly, because this model shows a number of features relevant for the present studies, such as the generating of the vacuum energy which is not associated with any propagating degrees of freedom (the Topological Casimir Effect). Furthermore, it may also exhibit non-static TCE discussed in section IIID when the radiation of real particles occurs as a result of a time dependent background, which could have profound consequences for cosmology, as we already mentioned in concluding section IV.

The "deformed QCD" model is a weakly coupled gauge theory, when the analytical computations can be performed in theoretically controllable way. Though it is a weakly coupled gauge theory, it nevertheless preserves all the crucial elements of strongly interacting QCD, including confinement, nontrivial $\theta$ dependence, degeneracy of the topological sectors, etc. Furthermore, it has been claimed [20] that there is no any phase transition in passage from weakly coupled deformed QCD to strongly coupled QCD, such that this model allows to address and answer a number of highly non-trivial questions. Important for the present work topic which will be reviewed here is the classification of the ground states in this system.

As described in [20] the proper infrared description of the theory is a dilute gas of $N$ types of monopoles characterized by their fractional topological charge $1 / N$ and magnetic charges, which are proportional to the simple roots and affine root $\alpha_{a} \in \Delta_{\text {aff }}$ of the Lie algebra of the $S U(N)$ gauge group. One can explicitly construct [21] the creation operator $\mathcal{M}_{a}(\mathbf{x})=e^{i \alpha_{a} \cdot \boldsymbol{\sigma}(\mathbf{x})}$ for a monopole of type $a$ at point $\mathbf{x}$, and compute its expectation value with the following result [22]:

$$
\left\langle\mathcal{M}_{a}(\mathbf{x})\right\rangle_{m}=\exp \left[i \frac{\theta+2 \pi m}{N}\right],
$$

where $m$ is integer which classifies a specific ground state. These distinct vacuum states classified by $m$ are not degenerate as it normally happens in supersymmetric field theories. Nevertheless, some degree of degeneracy (cor- responding to integers $m$ and $-m$ for $\theta=0$ ) still holds. However, these states, being the local minima of the system are metastable states, rather than absolutely stable states, because they can decay to the ground state $m=0$ through the tunnelling transition. The corresponding decay rate can be expressed in terms of the of the domain walls which separate these vacua [22]. There is a large number of different types of domain walls in the system. Their classification is uniquely fixed by the classification of corresponding vacua (A2).

It is also instructive to observe how the confinement in the vacuum states (A2) is realized. It turns out that the confinement in this system is a result of the condensation of the fractionally charged monopoles, as usual for $\theta=0, m=0$. However, the corresponding vacuum structure for $m \neq 0$ is less trivial as it represents a specific superposition of $N$ different monopole condensates. To be more specific: the condensation of the $N$ different monopole types is described by the same $N$ component $\boldsymbol{\sigma}(\mathbf{x})$ field which describes the confinement for $m=0$ state. However, different monopole condensates form a coherent superposition [22] shifted by a phase such that the corresponding magnetization $\left\langle\mathcal{M}_{a}(\mathbf{x})\right\rangle_{m}$ receives a non-trivial phase (A2) for each specific superposition. In other words, different vacuum states classified by parameter $m$ from (A2) correspond to the different superpositions of $N$ different monopole condensates ${ }^{5}$, while the effective long range field describing the dynamics of these monopolies, the $\boldsymbol{\sigma}(\mathbf{x})$-field, remains always the same for all $m$. This picture resembles in many ways the picture of the so-called oblique confinement suggested long ago by 't Hooft [23] for some special values of the $\theta_{m}=\frac{2 \pi m}{N}$.

Our final remark of this Appendix is that the Maxwell gauge system defined on a compact manifold and studied

\footnotetext{
5 It is often (wrongly) interpreted that the confinement for metastable vacuum states is due to the condensation of the dyons carrying the electric charges along with the magnetic charges. This (incorrect) interpretation based on observation that the topological density operator is the product of electric and magnetic fields, $\vec{E} \cdot \vec{B}$. One should remember, however, that the monopoles in this construction are pseudoparticles living in $4 \mathrm{~d}$ Euclidean space-time, rather than static $3 \mathrm{~d}$ objects. The finite action and finite topological charge $1 / N$ for these objects is a result of wrapping of the monopole's path along the Euclidean time direction $\mathbb{S}^{1}$ with nontrivial holonomy. These objects do not carry a conventional static electric charge; nevertheless, they do carry the topological charges defined in $4 \mathrm{~d}$ Euclidean space-time.

There is no fundamental difference between the vacuum structures for the metastable states and the lowest energy state. In all cases the confinement is realized as a condensation of the same monopoles, not dyons (by the reasons mentioned above it is more appropriate to use term "percolation" rather than "condensation" which is normally used for truly static 3 d objects in condensed matter physics). The only difference in structure between the metastable and the ground states is that $N$ different monopole's condensates describing a metastable vacuum state is represented by a superposition which includes a relative phase shift between different condensates, in contrast with the superposition which describes the ground vacuum state when this phase identically vanishes, see [22] for the details.
} 
in the present work, as well as non-abelian gauge "deformed QCD" model reviewed in this Appendix, show a number of other features (along with property of degeneracy already mentioned here) which are inherent characteristics of the topologically ordered phases. We refer to $[2,3]$ for corresponding arguments related to the $U(1)$ Maxwell gauge system formulated on a compact manifold, and to [24] with corresponding arguments applied to the $S U(N)$ "deformed QCD" model for the details.
[1] C. Cao, M. van Caspel and A. R. Zhitnitsky, Phys. Rev. D 87, 105012 (2013) [arXiv:1301.1706 [hep-th]].

[2] A. R. Zhitnitsky, Phys. Rev. D 88, 105029 (2013) [arXiv:1308.1960 [hep-th]].

[3] A. Zhitnitsky, Phys. Rev. D 90, 105007 (2014) [arXiv:1407.3804 [hep-th]].

[4] H. B. G. Casimir, Kon. Ned. Akad. Wetensch. Proc. 51, 793 (1948).

[5] G. Y. Cho and J. E. Moore, Annals Phys. 326, 1515 (2011) [arXiv:1011.3485 [cond-mat.str-el]].

[6] X. -G. Wen, arXiv:1210.1281 [cond-mat.str-el].

[7] S. Sachdev, arXiv:1203.4565 [hep-th].

[8] A. Cortijo, F. Guinea and M. A. H. Vozmediano, J. Phys. A 45, 383001 (2012) [arXiv:1112.2054 [cond-mat.meshall]].

[9] G. E. Volovik, Lecture Notes in Physics, 870, 343 (2013) [arXiv:1111.4627 [hep-ph]].

[10] G. T. Moore, J. Math. Phys. 11, 2679 (1970);

S. A. Fulling and P. C. W. Davies, Proc. R. Soc. Lond. A 348, 393 (1976);

P. C. Davies and S. A. Fulling, Proc. Roy. Soc. Lond. A 356, 237 (1977).

[11] G. Barton and C. Eberlein, Ann. Phys. 227, 222 (1993); M. Kardar et al., Rev. Mod. Phys. 71, 1233 (1999); V. V. Dodonov, pp. 309 in Modern Nonlinear Optics, Part 3, ed. M. W. Evans, Adv. Chem. Phys. Series, Vol. 119 (Wiley, New York, 2001).

[12] C. M. Wilson, G. Johansson, A. Pourkabirian, M. Simoen, J. R. Johansson, T. Duty, F. Nori and P. Delsing, Nature, 479, 376 (2011);

P.Lahteenmaki, G.S.Paraoanu, J.Hassel, and P.J.Hakonen, Proc. Natl. Acad. Sci. U.S.A. 110, 4234 (2013).
[13] M. Buttiker, Y. Imry, and R. Landauer, Phys. Lett. A 96, 365 (1983);

I. O. Kulik, Pis'ma Zh. Eksp. Teor. Fiz. 11, 407 (1970); JETP Lett. 11, 275 (1970).

[14] Y. Imry, Introduction to Mesoscopic Physics, 2nd ed. (Oxford University Press, New York, 2008);

E. Akkermans and G. Montambaux, Mesoscopic Physics of Electrons and Photons, reissue ed. (Cambridge University Press, 2011);

William Ennis Shanks, [arXiv:1112.3395 [cond-matt.meshall]].

[15] V. Chandrasekhar, R. A. Webb, M. J. Brady, M. B. Ketchen, W. J. Gallagher, and A. Kleinsasser, Phys. Rev. Lett., 67, 3578 (1991).

[16] M. Tsubota, K. Inagaki, and S. Tanda EPL 97, 57011 (2012)[arXiv:0906.5206 [cond-matt.mes-hall]].

[17] A. R. Zhitnitsky, Phys. Rev. D 89, 063529 (2014) [arXiv:1310.2258 [hep-th]].

[18] A. R. Zhitnitsky, Phys. Rev. D 90, 043504 (2014) [arXiv:1404.5965 [hep-ph]].

[19] D. Gaiotto, A. Kapustin, N. Seiberg and B. Willett, "Generalized Global Symmetries," arXiv:1412.5148 [hepth].

[20] M. Unsal and L. G. Yaffe, Phys. Rev. D 78, 065035 (2008) [arXiv:0803.0344 [hep-th]].

[21] E. Thomas and A. R. Zhitnitsky, Phys. Rev. D 85, 044039 (2012) [arXiv:1109.2608 [hep-th]].

[22] A. Bhoonah, E. Thomas and A. R. Zhitnitsky, Nucl. Phys. B 890, 30 (2015) [arXiv:1407.5121 [hep-ph]].

[23] G. 't Hooft, Nucl. Phys. B 153 , 141 (1979), G. 't Hooft, Nucl. Phys. B 190, 455 (1981).

[24] A. R. Zhitnitsky, Annals Phys. 336, 462 (2013) [arXiv:1301.7072 [hep-ph]]. 